quatrième série-tome 45 fascicule 3 mai-juin 2012

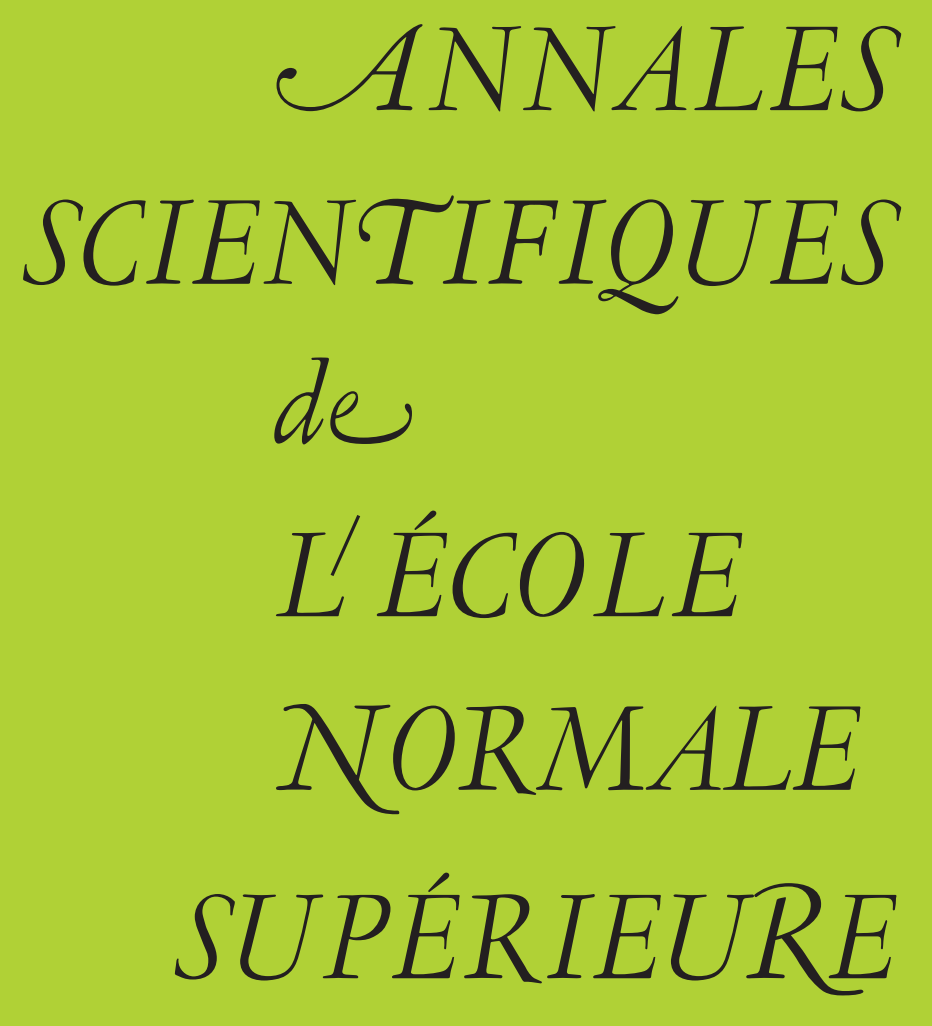

Cinzia CASAGRANDE

On the Picard number of divisors in Fano manifolds 
Ann. Scient. Éc. Norm. Sup.

$4^{\text {e }}$ série, t. 45, 2012, p. 363 à 403

\title{
ON THE PICARD NUMBER OF DIVISORS IN FANO MANIFOLDS
}

\author{
BY Cinzia CASAGRANDE
}

Abstract. - Let $X$ be a complex Fano manifold of arbitrary dimension, and $D$ a prime divisor in $X$. We consider the image $\mathcal{N}_{1}(D, X)$ of $\mathcal{N}_{1}(D)$ in $\mathcal{N}_{1}(X)$ under the natural push-forward of 1-cycles. We show that $\rho_{X}-\rho_{D} \leq \operatorname{codim} \mathcal{N}_{1}(D, X) \leq 8$. Moreover if $\operatorname{codim} \mathcal{N}_{1}(D, X) \geq 3$, then either $X \cong S \times T$ where $S$ is a Del Pezzo surface, or codim $\mathcal{N}_{1}(D, X)=3$ and $X$ has a fibration in Del Pezzo surfaces onto a Fano manifold $T$ such that $\rho_{X}-\rho_{T}=4$.

RÉSUMÉ. - Soient $X$ une variété de Fano lisse et complexe de dimension arbitraire, et $D$ un diviseur premier dans $X$. Nous considérons l'image $\mathcal{N}_{1}(D, X)$ de $\mathcal{N}_{1}(D)$ dans $\mathcal{N}_{1}(X)$ par l'application naturelle de push-forward de 1-cycles. Nous démontrons que $\rho_{X}-\rho_{D} \leq \operatorname{codim} \mathcal{N}_{1}(D, X) \leq 8$. De plus, si $\operatorname{codim} \mathcal{N}_{1}(D, X) \geq 3$, alors soit $X \cong S \times T$ où $S$ est une surface de Del Pezzo, soit $\operatorname{codim} \mathcal{N}_{1}(D, X)=3$ et $X$ a une fibration en surfaces de Del Pezzo sur une variété de Fano lisse $T$, telle que $\rho_{X}-\rho_{T}=4$.

\section{Introduction}

Let $X$ be a complex Fano manifold of arbitrary dimension $n$, and consider a prime divisor $D \subset X$. We denote by $\mathcal{N}_{1}(X)$ the $\mathbb{R}$-vector space of one-cycles in $X$, with real coefficients, modulo numerical equivalence; its dimension is the Picard number $\rho_{X}$ of $X$, and similarly for $D$. The inclusion $i: D \hookrightarrow X$ induces a push-forward of one-cycles $i_{*}: \mathcal{N}_{1}(D) \rightarrow \mathcal{N}_{1}(X)$, that does not need to be injective nor surjective. We are interested in the image

$$
\mathcal{N}_{1}(D, X):=i_{*}\left(\mathcal{N}_{1}(D)\right) \subseteq \mathcal{N}_{1}(X),
$$

which is the linear subspace of $\mathcal{N}_{1}(X)$ spanned by numerical classes of curves contained in $D$. The codimension of $\mathcal{N}_{1}(D, X)$ in $\mathcal{N}_{1}(X)$ is equal to the dimension of the kernel of the restriction $H^{2}(X, \mathbb{R}) \rightarrow H^{2}(D, \mathbb{R})$.

If $X$ is a surface, then it follows from the classification of Del Pezzo surfaces that $\operatorname{codim} \mathcal{N}_{1}(D, X)=\rho_{X}-1 \leq 8$. Our main result is that the same holds in any dimension. 
Theorem 1.1. - Let $X$ be a Fano manifold of dimension n. For every prime divisor $D \subset X$, we have

$$
\rho_{X}-\rho_{D} \leq \operatorname{codim} \mathcal{N}_{1}(D, X) \leq 8 .
$$

Moreover, suppose that there exists a prime divisor $D$ with $\operatorname{codim} \mathcal{N}_{1}(D, X) \geq 3$. Then one of the following holds:

(i) $X \cong S \times T$, where $S$ is a Del Pezzo surface with $\rho_{S} \geq \operatorname{codim} \mathcal{N}_{1}(D, X)+1$, and $D$ dominates $T$ under the projection;

(ii) $\operatorname{codim} \mathcal{N}_{1}(D, X)=3$ and there exists a flat surjective morphism $\varphi: X \rightarrow T$, with connected fibers, where $T$ is an $(n-2)$-dimensional Fano manifold, and $\rho_{X}-\rho_{T}=4$.

When $n \geq 4$ and $D$ is ample, one has $\mathcal{N}_{1}(D, X)=\mathcal{N}_{1}(X)$ and also $\operatorname{dim} \mathcal{N}_{1}(D, X)=\rho_{D}$ by Lefschetz Theorems on hyperplane sections, see [17, Example 3.1.25]. However in general $\operatorname{dim} \mathcal{N}_{1}(D, X)$ can be smaller than $\rho_{X}$ : for instance, if $D \cong \mathbb{P}^{n-1}$ is the exceptional divisor of the blow-up $X$ of any projective manifold at a point, we have $\rho_{D}=\operatorname{dim} \mathcal{N}_{1}(D, X)=1<\rho_{X}$.

In case (ii) of Theorem 1.1 the variety $X$ does not need to be a product of lower dimensional varieties, see Example 3.4.

Theorem 1.1 generalizes an analogous result in [9] for toric Fano varieties, obtained in a completely different way, using combinatorial techniques.

We recall that the pseudo-index of a Fano manifold $X$ is

$$
\iota_{X}=\min \left\{-K_{X} \cdot C \mid C \text { is a rational curve in } X\right\},
$$

and is a multiple of the index of $X$; one expects that Fano manifolds with large pseudo-index are simpler. When $\iota_{X}>1$ (i.e., when $X$ does not contain rational curves of anticanonical degree one), we show a stronger version of Theorem 1.1.

Theorem 1.2. - Let $X$ be a Fano manifold with pseudo-index $\iota_{X}>1$. For every prime divisor $D \subset X$, we have codim $\mathcal{N}_{1}(D, X) \leq 1$. More precisely, one of the following holds:

(i) $\iota_{X}=2$ and there exists a smooth morphism $\varphi: X \rightarrow Y$ with fibers isomorphic to $\mathbb{P}^{1}$, where $Y$ is a Fano manifold with $\iota_{Y}>1$;

(ii) for every prime divisor $D \subset X$, we have $\mathcal{N}_{1}(D, X)=\mathcal{N}_{1}(X), \rho_{X} \leq \rho_{D}$, and the restriction $H^{2}(X, \mathbb{R}) \rightarrow H^{2}(D, \mathbb{R})$ is injective. Moreover for every pair of prime divisors $D_{1}, D_{2}$ in $X$, we have $D_{1} \cap D_{2} \neq \varnothing$.

The author was led to this subject by the study of Fano manifolds with large Picard number (see [10] for an account of this problem). Let us mention two straightforward consequences of Theorem 1.1, which give bounds on $\rho_{X}$ in some good situations. The first concerns the case $\operatorname{dim} X \leq 5$, while the second is about Fano manifolds having a morphism onto a curve.

COROLlary 1.3. - Let $X$ be a Fano manifold, and suppose that there exists a prime divisor $D \subset X$ such that codim $\mathcal{N}_{1}(D, X) \geq 3$.

If $\operatorname{dim} X=4$ then either $\rho_{X} \leq 6$, or $X$ is a product of Del Pezzo surfaces and $\rho_{X} \leq 18$.

If $\operatorname{dim} X=5$ then either $\rho_{X} \leq 9$, or $X$ is a product and $\rho_{X} \leq 19$. 
Corollary 1.4. - Let $X$ be a Fano manifold, $\varphi: X \rightarrow \mathbb{P}^{1}$ a surjective morphism with connected fibers, and $F \subset X$ a general fiber. Then $\rho_{X} \leq \rho_{F}+8$.

Moreover if $\rho_{X} \geq \rho_{F}+4$, then $X \cong S \times T$ where $S$ is a Del Pezzo surface, $\varphi$ factors through the projection $X \rightarrow S$, and $F \cong \mathbb{P}^{1} \times T$.

Finally, we notice that some of the properties given by Theorem 1.1 are inherited by varieties dominated by a Fano manifold. We give two applications, and refer the reader to Lemma 4.1 for a more general statement.

Corollary 1.5. - Let $X$ be a Fano manifold and $\varphi: X \rightarrow Y$ a surjective morphism. Suppose that there exists a prime divisor $D \subset X$ such that $\operatorname{dim} \varphi(D) \leq 1$ (this always holds if $\operatorname{dim} Y=2$ ). Then $\rho_{Y} \leq 9$.

Moreover if $\rho_{Y} \geq 5$ then $\operatorname{dim} Y \leq 3$ and $X \cong S \times T$, where $S$ is a Del Pezzo surface.

COROLlary 1.6. - Let $X$ be a Fano manifold and $\varphi: X \rightarrow Y$ a surjective morphism with $\operatorname{dim} Y=3$. Then $\rho_{Y} \leq 10$.

Moreover if $\rho_{Y} \geq 6$ then $X \cong S \times T$ where $S$ is a Del Pezzo surface, $T$ has a contraction onto $\mathbb{P}^{1}$, and $\varphi$ factors through $X \rightarrow S \times \mathbb{P}^{1}$.

\section{Outline of the paper}

The idea that a special divisor should affect the geometry of $X$ is classical. In [6] Fano manifolds containing a divisor $D \cong \mathbb{P}^{n-1}$ with normal bundle $\mathcal{N}_{D / X} \cong \theta_{\mathbb{P}^{n-1}}(-1)$ are classified. This classification has been extended in [20] to the case $\mathcal{N}_{D / X} \cong \vartheta_{\mathbb{P}^{n-1}}(-a)$ with $a>0$; moreover [20, Proposition 5] shows that if $X$ contains a divisor $D$ with $\rho_{D}=1$, then $\rho_{X} \leq 3$. More generally, divisors $D \subset X$ with $\operatorname{dim} \mathcal{N}_{1}(D, X)=1$ or 2 play an important role in $[10,11]$.

In Section 2 we treat the main construction that will be used in the paper, based on the analysis of a Mori program for $-D$, where $D \subset X$ is a prime divisor; this is a development of a technique used in [11]. Let us give an idea of our approach, referring the reader to Section 2 for more details.

After [5, 13], we know that we can run a Mori program for any divisor in a Fano manifold $X$. In fact we need to consider special Mori programs, where all involved extremal rays have positive intersection with the anticanonical divisor (see Section 2.1).

Then, given a prime divisor $D \subset X$, we consider a special Mori program for $-D$, which roughly means that we contract or flip extremal rays having positive intersection with $D$, until we get a fiber type contraction such that (the transform of) $D$ dominates the target.

If $c:=\operatorname{codim} \mathcal{N}_{1}(D, X)>0$, by studying how the codimension of $\mathcal{N}_{1}(D, X)$ varies under the birational maps and the related properties of the extremal rays, we obtain $c-1$ pairwise disjoint prime divisors $E_{1}, \ldots, E_{c-1} \subset X$, all intersecting $D$, such that each $E_{i}$ is a smooth $\mathbb{P}^{1}$-bundle with $E_{i} \cdot f_{i}=-1$, where $f_{i} \subset E_{i}$ is a fiber (see Proposition 2.5 and Lemma 2.7). We call $E_{1}, \ldots, E_{c-1}$ the $\mathbb{P}^{1}$-bundles determined by the special Mori program for $-D$ that we are considering; they play an essential role throughout the paper.

We conclude Section 2 proving Theorem 1.2 about the case with pseudo-index $\iota_{X}>1$.

In Section 3 we consider the following invariant of $X$ :

$$
c_{X}:=\max \left\{\operatorname{codim} \mathcal{N}_{1}(D, X) \mid D \text { is a prime divisor in } X\right\} .
$$


In terms of this invariant, our main result is that $c_{X} \leq 8$, and if $c_{X} \geq 3$, then either $X$ is a product, or $c_{X}=3$ and $X$ has a flat fibration onto an $(n-2)$-dimensional Fano manifold (see Theorem 3.3 for a precise statement). The proof of this result is quite long: it takes the whole Section 3, and is divided in several steps; see 3.5 for a plan. The strategy is to apply the construction of Section 2 to prime divisors of "minimal Picard number", i.e., with codim $\mathcal{N}_{1}(D, X)=c_{X}$. We show that there exists a prime divisor $E_{0}$ with $\operatorname{codim} \mathcal{N}_{1}\left(E_{0}, X\right)=c_{X}$, such that $E_{0}$ is a smooth $\mathbb{P}^{1}$-bundle with $E_{0} \cdot f_{0}=-1$, where $f_{0} \subset E_{0}$ is a fiber. Applying the previous results to $E_{0}$, we obtain a bunch of disjoint divisors with a $\mathbb{P}^{1}$-bundle structure, and we use them to show that $X$ is a product, or to construct a fibration in Del Pezzo surfaces.

Finally in Section 4 we use this result (Theorem 3.3) to prove the remaining results stated above: Theorem 1.1 and its Corollaries 1.3 to 1.6.

\section{Acknowledgements}

I am grateful to Tommaso de Fernex for an important suggestion concerning Proposition 2.4.

This paper was written mainly during a visit to the Mathematical Sciences Research Institute in Berkeley, for the program in Algebraic Geometry in spring 2009. I would like to thank MSRI for the kind hospitality, and GNSAGA-INdAM and the Research Project "Geometria delle varietà algebriche e dei loro spazi di moduli" (PRIN 2006) for financial support.

\section{Notation and terminology}

We work over the field of complex numbers. A manifold is a smooth variety. A $\mathbb{P}^{1}$-bundle is a projectivization of a rank 2 vector bundle.

Let $X$ be a projective variety.

$\mathcal{N}_{1}(X)$ (respectively, $\mathcal{N}^{1}(X)$ ) is the $\mathbb{R}$-vector space of one-cycles (respectively, Cartier divisors) with real coefficients, modulo numerical equivalence.

$[C]$ is the numerical equivalence class in $\mathcal{N}_{1}(X)$ of a curve $C \subset X ;[D]$ is the numerical equivalence class in $\mathcal{N}^{1}(X)$ of a $\mathbb{Q}$-Cartier divisor $D$ in $X$.

If $E \subset X$ is an irreducible closed subset and $C \subset E$ is a curve, $[C]_{E}$ is the numerical equivalence class of $C$ in $\mathcal{N}_{1}(E)$.

The symbol $\equiv$ stands for numerical equivalence (for both 1-cycles and $\mathbb{Q}$-Cartier divisors).

For any $\mathbb{Q}$-Cartier divisor $D$ in $X, D^{\perp}:=\left\{\gamma \in \mathcal{N}_{1}(X) \mid D \cdot \gamma=0\right\}$.

$\mathrm{NE}(X) \subset \mathcal{N}_{1}(X)$ is the convex cone generated by classes of effective curves, and $\overline{\mathrm{NE}}(X)$ is its closure.

An extremal ray $R$ of $X$ is a one-dimensional face of $\overline{\mathrm{NE}}(X)$; $\operatorname{Locus}(R) \subseteq X$ is the union of all curves whose class is in $R$.

If $R$ is an extremal ray of $X$ and $D$ is a $\mathbb{Q}$-Cartier divisor in $X$, we say that $D \cdot R>0$, respectively $D \cdot R=0$, etc. if for $\gamma \in R \backslash\{0\}$ we have $D \cdot \gamma>0$, respectively $D \cdot \gamma=0$, etc.

Assume that $X$ is normal.

A contraction of $X$ is a surjective morphism with connected fibers $\varphi: X \rightarrow Y$, where $Y$ is normal and projective; $\mathrm{NE}(\varphi)$ is the face of $\overline{\mathrm{NE}}(X)$ generated by classes of curves contracted by $\varphi$.

4 e SÉRIE - TOME $45-2012$ - No 3 
A contraction $\varphi: X \rightarrow Y$ is elementary if $\rho_{X}-\rho_{Y}=1$.

We say that an elementary contraction $\varphi: X \rightarrow Y$ (or the extremal ray $\mathrm{NE}(\varphi)$ ) is of type $(n-1, n-2)^{s m}$ if it is the blow-up of a smooth codimension 2 subvariety contained in the smooth locus of $Y$ (here $n=\operatorname{dim} X$ ).

If $Z \subseteq X$ is a closed subset and $i: Z \hookrightarrow X$ is the inclusion, we set

$$
\mathcal{N}_{1}(Z, X):=i_{*}\left(\mathcal{N}_{1}(Z)\right) \subseteq \mathcal{N}_{1}(X) \text { and } \mathrm{NE}(Z, X):=i_{*}(\mathrm{NE}(Z)) \subseteq \mathrm{NE}(X) \subset \mathcal{N}_{1}(X) \text {. }
$$

\section{Mori programs and prime divisors}

\subsection{Special Mori programs in Fano manifolds}

In this section we recall what a Mori program is, and explain that by [13] and [5] we can run a Mori program for any divisor on a Fano manifold. We also introduce and show the existence of "special Mori programs", where all involved extremal rays have positive intersection with the anticanonical divisor.

We begin by recalling the following fundamental result.

Theorem 2.1 ([5], Corollary 1.3.2). - Any Fano manifold is a Mori dream space.

We refer the reader to [13] for the definition and properties of a Mori dream space; in particular, a Mori dream space is always a normal and $\mathbb{Q}$-factorial projective variety. We also need the following.

Proposition 2.2 ([13], Proposition 1.11(1)). - Let $X$ be a Mori dream space and $B$ a divisor in $X$. Then there exists a finite sequence

$$
X=X_{0} \stackrel{\sigma_{0}}{\rightarrow} X_{1} \rightarrow \quad \cdots \quad \rightarrow X_{k-1} \stackrel{\sigma_{k-1}}{\rightarrow-} X_{k}
$$

such that:

- every $X_{i}$ is a normal and $\mathbb{Q}$-factorial projective variety;

- for every $i=0, \ldots, k-1$ there exists an extremal ray $Q_{i}$ of $X_{i}$ such that $B_{i} \cdot Q_{i}<0$, where $B_{i} \subset X_{i}$ is the transform ${ }^{(1)}$ of $B$, Locus $\left(Q_{i}\right) \subsetneq X_{i}$, and $\sigma_{i}$ is either the contraction of $Q_{i}$ (if $Q_{i}$ is divisorial), or its flip (if $Q_{i}$ is small);

- either $B_{k}$ is nef, or there exists an extremal ray $Q_{k}$ in $X_{k}$, with a fiber type contraction $\varphi: X_{k} \rightarrow Y$, such that $B_{k} \cdot Q_{k}<0$.

Moreover, the choice of the extremal rays $Q_{i}$ is arbitrary among those that have negative intersection with $B_{i}$.

A sequence as above is called a Mori program for the divisor $B$. We refer the reader to [16, Definition 6.5] for the definition of flip.

An important remark is that when $X$ is Fano, there is always a suitable choice of a Mori program where all involved extremal rays have positive intersection with the anticanonical divisor.

(1) More precisely, $B_{i}$ is the transform of $B_{i-1}$ if $\sigma_{i-1}$ is a flip, and $B_{i}=\left(\sigma_{i-1}\right)_{*}\left(B_{i-1}\right)$ if $\sigma_{i-1}$ is a divisorial contraction. 
Proposition 2.4. - Let $X$ be a Fano manifold and $B$ a divisor on $X$. Then there exists a Mori program for $B$ as (2.3), such that $-K_{X_{i}} \cdot Q_{i}>0$ for every $i=0, \ldots, k$. We call such a sequence a special Mori program for $B$.

This is a very special case of the MMP with scaling, see [5, Remark 3.10.9]. For the reader's convenience, we give a proof. The idea is to choose a facet of the cone of nef divisors $\operatorname{Nef}(X) \subset \mathcal{N}^{1}(X)$ met by moving from $[B]$ to $\left[-K_{X}\right]$ along a line in $\mathcal{N}^{1}(X)$, and to repeat the same at each step.

Proof of Proposition 2.4. - By Theorem 2.1 $X$ is a Mori dream space, therefore Proposition 2.2 applies to $X$, and there exists a Mori program for $B$. We have to prove that we can choose $Q_{0}, \ldots, Q_{k}$ with $B_{i} \cdot Q_{i}<0$ and $-K_{X_{i}} \cdot Q_{i}>0$ for all $i=0, \ldots, k$.

We can assume that $B$ is not nef. Set

$$
\lambda_{0}:=\sup \left\{\lambda \in \mathbb{R} \mid(1-\lambda)\left(-K_{X}\right)+\lambda B \text { is nef }\right\},
$$

so that $\lambda_{0} \in \mathbb{Q}, 0<\lambda_{0}<1$, and $H_{0}:=\left(1-\lambda_{0}\right)\left(-K_{X}\right)+\lambda_{0} B$ is nef but not ample. Then there exists an extremal ray $Q_{0}$ of $\mathrm{NE}(X)$ such that $H_{0} \cdot Q_{0}=0$ and $B \cdot Q_{0}<0$; in particular, $-K_{X} \cdot Q_{0}>0$.

If $Q_{0}$ is of fiber type, we are done. Otherwise, let $\sigma_{0}: X \rightarrow X_{1}$ be either the contraction of $Q_{0}$ (if divisorial), or its flip (if small), and let $B_{1}$ be the transform of $B$. Then $\left(1-\lambda_{0}\right)\left(-K_{X_{1}}\right)+\lambda_{0} B_{1}$ is nef in $X_{1}$.

If $B_{1}$ is nef we are done. If not, we set

$$
\lambda_{1}:=\sup \left\{\lambda \in \mathbb{R} \mid(1-\lambda)\left(-K_{X_{1}}\right)+\lambda B_{1} \text { is nef }\right\},
$$

so that $\lambda_{1} \in \mathbb{Q}, \lambda_{0} \leq \lambda_{1}<1$, and $H_{1}:=\left(1-\lambda_{1}\right)\left(-K_{X_{1}}\right)+\lambda_{1} B_{1}$ is nef but not ample. There exists an extremal ray $Q_{1}$ of $\mathrm{NE}\left(X_{1}\right)$ such that $H_{1} \cdot Q_{1}=0$ and $B_{1} \cdot Q_{1}<0$, hence $-K_{X_{1}} \cdot Q_{1}>0$. Now we iterate the procedure.

\subsection{Running a Mori program for $-D$}

In this section we study in detail what happens when we run a Mori program for $-D$, where $D$ is a prime divisor. This point of view has already been considered in [11], and is somehow opposite to the classical one: we consider extremal rays having positive intersection with $D$. In particular, we are interested in how the number $\operatorname{codim} \mathcal{N}_{1}(D, X)$ varies under the Mori program.

We first describe the general situation for a prime divisor $D$ in a Mori dream space (Lemma 2.6), and then consider the case of a special Mori program for $-D$ where $D$ is a prime divisor in a Fano manifold (Lemma 2.7). In particular, we will show the following.

Proposition 2.5. - Let $X$ be a Fano manifold and $D \subset X$ a prime divisor. Suppose that $\operatorname{codim} \mathcal{N}_{1}(D, X)>0$.

Then there exist pairwise disjoint smooth prime divisors $E_{1}, \ldots, E_{s} \subset X$, with $s=\operatorname{codim} \mathcal{N}_{1}(D, X)-1$ or $s=\operatorname{codim} \mathcal{N}_{1}(D, X)$, such that every $E_{j}$ is a $\mathbb{P}^{1}$-bundle with $E_{j} \cdot f_{j}=-1$, where $f_{j} \subset E_{j}$ is a fiber; moreover $D \cdot f_{j}>0$ and $\left[f_{j}\right] \notin \mathcal{N}_{1}(D, X)$. In particular $E_{j} \cap D \neq \varnothing$ and $E_{j} \neq D$. 
It is important to point out that the $\mathbb{P}^{1}$-bundles $E_{1}, \ldots, E_{s}$ are determined not only by $D$, but by the choice of a special Mori program for $-D$ (see Lemma 2.7). In fact the divisors $E_{j}$ are the transforms of the loci of some of the extremal rays of the Mori program, the ones where $\operatorname{codim} \mathcal{N}_{1}(D, X)$ drops.

Finally we study in more detail the case where $s=\operatorname{codim} \mathcal{N}_{1}(D, X)-1$ in the Proposition above; in this situation we show that there is an open subset of $X$ which has a conic bundle structure (see Lemma 2.8).

We conclude the section with the proof of Theorem 1.2.

Lemma 2.6. - Let $X$ be a Mori dream space and $D \subset X$ a prime divisor. Consider a Mori program for $-D$ :

$$
X=X_{0} \stackrel{\sigma_{0}}{\rightarrow} X_{1} \rightarrow \rightarrow \quad \cdots \quad \rightarrow X_{k-1} \stackrel{\sigma_{k-1}}{\rightarrow} X_{k} .
$$

Let $D_{i} \subset X_{i}$ be the transform of $D$, for $i=1, \ldots, k$, and set $D_{0}:=D$, so that $D_{i} \cdot Q_{i}>0$ for $i=0, \ldots, k$. We have the following.

(1) Every $D_{i}$ is a prime divisor in $X_{i}$, and the program ends with an elementary contraction of fiber type $\varphi: X_{k} \rightarrow Y$ such that $\operatorname{NE}(\varphi)=Q_{k}$ and $\varphi\left(D_{k}\right)=Y$.

(2) $\#\left\{i \in\{0, \ldots, k\} \mid Q_{i} \not \subset \mathcal{N}_{1}\left(D_{i}, X_{i}\right)\right\}=\operatorname{codim} \mathcal{N}_{1}(D, X)$.

(3) Set $c_{i}:=\operatorname{codim} \mathcal{N}_{1}\left(D_{i}, X_{i}\right)$ for $i=0, \ldots, k$. For every $i=0, \ldots, k-1$ we have

$$
c_{i+1}=\left\{\begin{array}{ll}
c_{i} & \text { if } Q_{i} \subset \mathcal{N}_{1}\left(D_{i}, X_{i}\right) \\
c_{i}-1 & \text { if } Q_{i} \not \subset \mathcal{N}_{1}\left(D_{i}, X_{i}\right)
\end{array}, \text { and } c_{k}= \begin{cases}0 & \text { if } Q_{k} \subset \mathcal{N}_{1}\left(D_{k}, X_{k}\right) \\
1 & \text { if } Q_{k} \not \subset \mathcal{N}_{1}\left(D_{k}, X_{k}\right) .\end{cases}\right.
$$

(4) Suppose that $X$ is smooth. Let $A_{1} \subset X_{1}$ be the indeterminacy locus of $\sigma_{0}^{-1}$, and for $i=2, \ldots, k$, if $\sigma_{i-1}$ is a divisorial contraction (respectively, if $\sigma_{i-1}$ is a flip), let $A_{i} \subset X_{i}$ be the union of $\sigma_{i-1}\left(A_{i-1}\right)$ (respectively, the transform of $A_{i-1}$ ) and the indeterminacy locus of $\sigma_{i-1}^{-1}$.

Then for all $i=1, \ldots, k$ we have $\operatorname{Sing}\left(X_{i}\right) \subseteq A_{i} \subset D_{i}$, and the birational map $X_{i} \rightarrow X$ is an isomorphism over $X_{i} \backslash A_{i}$.

Proof. - Most of the statements are shown in [11] (see in particular Remarks 2.5 and 2.6, and Lemma 3.6); for the reader's convenience we give a proof. We have $D_{i} \cdot Q_{i}>0$ for every $i=0, \ldots, k$, just by the definition of Mori program for $-D$.

Let $i \in\{0, \ldots, k-1\}$ be such that $\sigma_{i}$ is a divisorial contraction. Then $D_{i} \neq \operatorname{Exc}\left(\sigma_{i}\right)$ (for otherwise $\left.D_{i} \cdot Q_{i}<0\right)$, hence $D_{i+1}=\sigma_{i}\left(D_{i}\right) \subset X_{i+1}$ is a prime divisor. On the other hand $D_{i}$ intersects every non-trivial fiber of $\sigma_{i}$ (because $D_{i} \cdot Q_{i}>0$ ), in particular $D_{i} \cap \operatorname{Exc}\left(\sigma_{i}\right) \neq \varnothing$ and $D_{i+1} \supset \sigma_{i}\left(\operatorname{Exc}\left(\sigma_{i}\right)\right)$. Notice that $\sigma_{i}\left(\operatorname{Exc}\left(\sigma_{i}\right)\right)$ is the indeterminacy locus of $\sigma_{i}^{-1}$.

Consider the push-forward $\left(\sigma_{i}\right)_{*}: \mathcal{N}_{1}\left(X_{i}\right) \rightarrow \mathcal{N}_{1}\left(X_{i+1}\right)$. We have $\operatorname{ker}\left(\sigma_{i}\right)_{*}=\mathbb{R} Q_{i}$ and $\mathcal{N}_{1}\left(D_{i+1}, X_{i+1}\right)=\left(\sigma_{i}\right)_{*}\left(\mathcal{N}_{1}\left(D_{i}, X_{i}\right)\right)$, therefore $c_{i+1}=c_{i}$ if $Q_{i} \subset \mathcal{N}_{1}\left(D_{i}, X_{i}\right)$, and $c_{i+1}=c_{i}-1$ otherwise.

Now let $i \in\{0, \ldots, k-1\}$ be such that $\sigma_{i}$ is a flip, and consider the standard flip diagram:

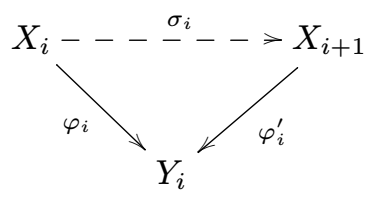


where $\varphi_{i}$ is the contraction of $Q_{i}$, and $\varphi_{i}^{\prime}$ is the corresponding small elementary contraction of $X_{i+1}$. We have $D_{i+1} \cdot \mathrm{NE}\left(\varphi_{i}^{\prime}\right)<0$, in particular $\operatorname{Exc}\left(\varphi_{i}^{\prime}\right) \subset D_{i+1}$ and $\operatorname{NE}\left(\varphi_{i}^{\prime}\right) \subset \mathcal{N}_{1}\left(D_{i+1}, X_{i+1}\right)$. Notice that $\operatorname{Exc}\left(\varphi_{i}^{\prime}\right)$ is the indeterminacy locus of $\sigma_{i}^{-1}$.

Moreover $\varphi_{i}\left(D_{i}\right)=\varphi_{i}^{\prime}\left(D_{i+1}\right)$, so that

$$
\left(\varphi_{i}\right)_{*}\left(\mathcal{N}_{1}\left(D_{i}, X_{i}\right)\right)=\mathcal{N}_{1}\left(\varphi_{i}\left(D_{i}\right), Y_{i}\right)=\left(\varphi_{i}^{\prime}\right)_{*}\left(\mathcal{N}_{1}\left(D_{i+1}, X_{i+1}\right)\right)
$$

Since $\operatorname{ker}\left(\varphi_{i}^{\prime}\right)_{*} \subseteq \mathcal{N}_{1}\left(D_{i+1}, X_{i+1}\right)$, we have $c_{i+1}=\operatorname{codim} \mathcal{N}_{1}\left(\varphi_{i}\left(D_{i}\right), Y_{i}\right)$. We deduce again that $c_{i+1}=c_{i}$ if $Q_{i} \subset \mathcal{N}_{1}\left(D_{i}, X_{i}\right)$, and $c_{i+1}=c_{i}-1$ otherwise.

In particular the preceding analysis shows that for every $i=1, \ldots, k$ the divisor $D_{i}$ contains the indeterminacy locus of $\sigma_{i}^{-1}$, so that $A_{i} \subset D_{i}$. By definition, $A_{i}$ contains the indeterminacy locus of the birational map $\left(\sigma_{i-1} \circ \cdots \circ \sigma_{0}\right)^{-1}: X_{i} \rightarrow X$; in particular $X_{i} \backslash A_{i}$ is isomorphic to an open subset of $X$, thus it is smooth if $X$ is smooth. This shows (4).

Consider now the prime divisor $D_{k} \subset X_{k}$. Clearly $-D_{k}$ cannot be nef, therefore the program ends with a fiber type contraction $\varphi: X_{k} \rightarrow Y$. Since $D_{k} \cdot Q_{k}>0, D_{k}$ intersects every fiber of $\varphi$, namely $\varphi\left(D_{k}\right)=Y$, and we have (1).

In particular $\varphi_{*}\left(\mathcal{N}_{1}\left(D_{k}, X_{k}\right)\right)=\mathcal{N}_{1}(Y)$, hence either $c_{k}=0$ (i.e., $\mathcal{N}_{1}\left(D_{k}, X_{k}\right)=$ $\left.\mathcal{N}_{1}\left(X_{k}\right)\right)$, or $c_{k}=1$ and $Q_{k} \not \subset \mathcal{N}_{1}\left(D_{k}, X_{k}\right)$. Thus we have (3), which implies directly (2).

Lemma 2.7. - Let $X$ be a Fano manifold and $D \subset X$ a prime divisor. Consider a special Mori program for $-D$ :

$$
X=X_{0} \stackrel{\sigma_{0}}{\rightarrow-} X_{1} \rightarrow \rightarrow \quad \cdots \quad \rightarrow X_{k-1} \stackrel{\sigma_{k-1}}{\rightarrow-\rightarrow} X_{k} .
$$

Then we have the following (we keep the notation of Lemma 2.6).

(1) Let $i \in\{0, \ldots, k-1\}$ be such that $Q_{i} \not \subset \mathcal{N}_{1}\left(D_{i}, X_{i}\right)$.

Then $Q_{i}$ is of type $(n-1, n-2)^{\text {sm }}$, i.e., $\sigma_{i}: X_{i} \rightarrow X_{i+1}$ is the blow-up of a smooth subvariety of codimension 2, contained in the smooth locus of $X_{i+1}$. Moreover $\operatorname{Exc}\left(\sigma_{i}\right) \cap A_{i}=\varnothing$, hence $\operatorname{Exc}\left(\sigma_{i}\right)$ does not intersect the loci of the birational maps $\sigma_{l}$ for $l<i$.

(2) Set $s:=\#\left\{i \in\{0, \ldots, k-1\} \mid Q_{i} \not \subset \mathcal{N}_{1}\left(D_{i}, X_{i}\right)\right\}$. We have two possibilities:

either $s=\operatorname{codim} \mathcal{N}_{1}(D, X)$ and $\mathcal{N}_{1}\left(D_{k}, X_{k}\right)=\mathcal{N}_{1}\left(X_{k}\right)$,

or $s=\operatorname{codim} \mathcal{N}_{1}(D, X)-1, Q_{k} \not \subset \mathcal{N}_{1}\left(D_{k}, X_{k}\right)$, and $\operatorname{codim} \mathcal{N}_{1}\left(D_{k}, X_{k}\right)=1$.

(3) Set $\left\{i_{1}, \ldots, i_{s}\right\}:=\left\{i \in\{0, \ldots, k-1\} \mid Q_{i} \not \subset \mathcal{N}_{1}\left(D_{i}, X_{i}\right)\right\}$, and let $E_{j} \subset X$ be the transform of $\operatorname{Exc}\left(\sigma_{i_{j}}\right) \subset X_{i_{j}}$ for every $j=1, \ldots, s$.

Then $E_{j}$ is a smooth $\mathbb{P}^{1}$-bundle, with fiber $f_{j} \subset E_{j}$, such that $E_{j} \cdot f_{j}=-1, D \cdot f_{j}>0$, and $\left[f_{j}\right] \notin \mathcal{N}_{1}(D, X)$. In particular $E_{j} \cap D \neq \varnothing$ and $E_{j} \neq D$.

(4) The prime divisors $E_{1}, \ldots, E_{s}$ are pairwise disjoint.

We call $E_{1}, \ldots, E_{s}$ the $\mathbb{P}^{1}$-bundles determined by the special Mori program for $-D$ that we are considering. These divisors will play a key role throughout the paper.

Notice that Proposition 2.5 is a straightforward consequence of Proposition 2.4 and of Lemma 2.7, more precisely of 2.7(3) and 2.7(4).

4 e SÉRIE - TOME $45-2012$ - No 3 
Proof. - Statement (1) follows from [11, Lemma 3.9].

By 2.6(2) we have

$$
s= \begin{cases}\operatorname{codim} \mathcal{N}_{1}(D, X) & \text { if } Q_{k} \subset \mathcal{N}_{1}\left(D_{k}, X_{k}\right), \\ \operatorname{codim} \mathcal{N}_{1}(D, X)-1 & \text { if } Q_{k} \not \subset \mathcal{N}_{1}\left(D_{k}, X_{k}\right) .\end{cases}
$$

Together with 2.6(3) this yields (2).

Let $j \in\{1, \ldots, s\}$. By (1) we have $E_{j} \cong \operatorname{Exc}\left(\sigma_{i_{j}}\right)$, thus $E_{j}$ is a smooth $\mathbb{P}^{1}$-bundle with $E_{j} \cdot f_{j}=-1$, where $f_{j} \subset E_{j}$ is a fiber, and $D \cdot f_{j}>0$ because $D_{i_{j}} \cdot Q_{i_{j}}>0$ in $X_{i_{j}}$. In particular $E_{j} \cap D \neq \varnothing$ and $E_{j} \neq D$. Moreover $\left[f_{j}\right] \subset \mathcal{N}_{1}(D, X)$ would yield $Q_{i_{j}} \subset \mathcal{N}_{1}\left(D_{i_{j}}, X_{i_{j}}\right)$, which is excluded by definition. Therefore we have (3).

Finally $E_{1}, \ldots, E_{s}$ are pairwise disjoint, because for $j=1, \ldots, s$ the divisor $\operatorname{Exc}\left(\sigma_{i_{j}}\right)$ does not intersect the loci of the previous birational maps.

Here is a more detailed description of the case where $s=\operatorname{codim} \mathcal{N}_{1}(D, X)-1$ in Lemma 2.7.

Lemma 2.8 (Conic bundle case). - Let $X$ be a Fano manifold and $D \subset X$ a prime divisor. Consider a special Mori program for $-D$; we keep the same notation as in Lemmas 2.6 and 2.7. Set $c:=\operatorname{codim} \mathcal{N}_{1}(D, X), \sigma:=\sigma_{k-1} \circ \cdots \circ \sigma_{0}: X \rightarrow X_{k}$, and $\psi:=\varphi \circ \sigma: X \rightarrow Y$.

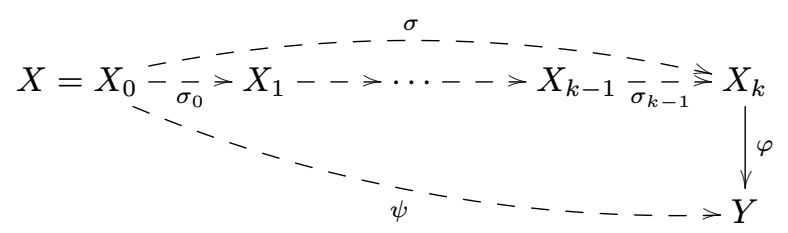

We assume that $Q_{k} \not \subset \mathcal{N}_{1}\left(D_{k}, X_{k}\right)$, equivalently that $s=c-1$ (see 2.7(2)). Then we have the following.

(1) Every fiber of $\varphi$ has dimension $1, \operatorname{dim} Y=n-1$, and $\varphi$ is finite on $D_{k}$.

(2) Let $j \in\{1, \ldots, c-1\}$ and consider $\sigma_{i_{j}}\left(\operatorname{Exc}\left(\sigma_{i_{j}}\right)\right) \subset X_{i_{j}+1}$. For every $m=i_{j}+1, \ldots, k-1$ the set $\operatorname{Locus}\left(Q_{m}\right) \subset X_{m}$ is disjoint from the image of $\sigma_{i_{j}}\left(\operatorname{Exc}\left(\sigma_{i_{j}}\right)\right)$ in $X_{m}$, so that the birational map $X_{i_{j}+1} \rightarrow X_{k}$ is an isomorphism on $\sigma_{i_{j}}\left(\operatorname{Exc}\left(\sigma_{i_{j}}\right)\right)$, and $\sigma$ is regular on $E_{j} \subset X$.

(3) There exist open subsets $U \subseteq X$ and $V \subseteq Y$, with $E_{1}, \ldots, E_{c-1} \subset U$, such that $V$ and $\varphi^{-1}(V)$ are smooth, $\varphi_{\mid \varphi^{-1}(V)}: \varphi^{-1}(V) \rightarrow V$ and $\psi: U \rightarrow V$ are conic bundles, and $\sigma_{\mid U}$ is the blow-up of pairwise disjoint smooth subvarieties $T_{1}, \ldots, T_{c-1} \subset \varphi^{-1}(V)$, of dimension $n-2$, with exceptional divisors $E_{1}, \ldots, E_{c-1}$.

$$
\underset{\sigma_{\mid U}}{\stackrel{\psi}{\longrightarrow} \varphi^{-1}(V) \underset{\varphi}{\longrightarrow}} V
$$

In particular we have $\operatorname{Locus}\left(Q_{m}\right) \subseteq X_{m} \backslash\left(\sigma_{m-1} \circ \cdots \circ \sigma_{0}\right)(U)$ for every $m \in\{0, \ldots, k-1\} \backslash\left\{i_{1}, \ldots, i_{c-1}\right\}$. 
(4) Set $Z_{j}:=\psi\left(E_{j}\right) \subset V$ for every $j \in\{1, \ldots, c-1\}$. Then $Z_{1}, \ldots, Z_{c-1} \subset Y$ are pairwise disjoint smooth prime divisors, and $\psi^{*}\left(Z_{j}\right)=E_{j}+\widehat{E}_{j}$, where $\widehat{E}_{j} \subset U$ is a smooth $\mathbb{P}^{1}$-bundle with fiber $\widehat{f}_{j} \subset \widehat{E}_{j}, f_{j}+\widehat{f}_{j}$ is numerically equivalent to a general fiber of $\psi$, and

$$
\widehat{E}_{j} \cdot \widehat{f}_{j}=-1, \quad E_{j} \cdot \widehat{f}_{j}=\widehat{E}_{j} \cdot f_{j}=1, \text { and }\left[\widehat{f}_{j}\right] \notin \mathcal{N}_{1}\left(E_{j}, X\right),
$$

for every $j \in\{1, \ldots, c-1\}$. In particular the divisors $D, E_{1}, \ldots, E_{c-1}, \widehat{E}_{1}, \ldots, \widehat{E}_{c-1}$ are all distinct, and $E_{1} \cup \widehat{E}_{1}, \ldots, E_{c-1} \cup \widehat{E}_{c-1}$ are pairwise disjoint.

We refer the reader to [9, p. 1478-1479] for an explicit description of the rational conic bundle $\psi$ in the toric case.

Proof of Lemma 2.8. - Let $F \subset X_{k}$ be a fiber of $\varphi$. Then $F \cap D_{k} \neq \varnothing$ because $D_{k} \cdot Q_{k}>0$; on the other hand $\operatorname{dim}\left(F \cap D_{k}\right)=0$, because if there exists a curve $C \subset F \cap D_{k}$, then $[C] \in Q_{k}$ and $[C] \in \mathcal{N}_{1}\left(D_{k}, X_{k}\right)$, thus $Q_{k} \subset \mathcal{N}_{1}\left(D_{k}, X_{k}\right)$ against our assumptions. Hence every fiber of $\varphi$ has dimension $1, \operatorname{dim} Y=n-1$, and we have (1).

Recall from 2.6(4) that $\operatorname{Sing}\left(X_{k}\right) \subseteq A_{k}$, and notice that codim $A_{k} \geq 2$, therefore $A_{k}$ cannot dominate $Y$. Restricting $\varphi$ we get a contraction $X_{k} \backslash \varphi^{-1}\left(\varphi\left(A_{k}\right)\right) \rightarrow Y \backslash \varphi\left(A_{k}\right)$ of a smooth variety, with $-K_{X_{k}}$ relatively ample (because $-K_{X_{k}} \cdot Q_{k}>0$ ), and one-dimensional fibers. We conclude that $Y \backslash \varphi\left(A_{k}\right)$ is smooth and that $\varphi_{\mid X_{k} \backslash \varphi^{-1}\left(\varphi\left(A_{k}\right)\right)}$ is a conic bundle (see [3, Theorem 4.1(2)]).

By 2.6(4), $\sigma: X \rightarrow X_{k}$ is an isomorphism over $X_{k} \backslash A_{k}$. If $U_{1}:=\sigma^{-1}\left(X_{k} \backslash \varphi^{-1}\left(\varphi\left(A_{k}\right)\right)\right)$, then $\psi: U_{1} \rightarrow Y \backslash \varphi\left(A_{k}\right)$ is again a conic bundle; in particular it is flat, and induces an injective morphism $\iota: Y \backslash \varphi\left(A_{k}\right) \rightarrow \operatorname{Hilb}(X)$. Let $H \subset \operatorname{Hilb}(X)$ be the closure of the image of $\iota$, and $\mathscr{C} \subset H \times X$ the restriction of the universal family over Hilb $(X)$. We get a diagram:

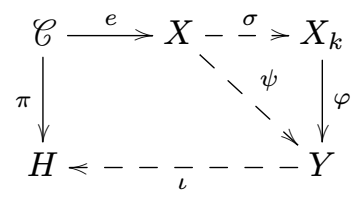

where $\pi: \mathscr{C} \rightarrow H$ and $e: \mathscr{C} \rightarrow X$ are the projections, and $\iota$ is birational. We want to compare the degenerations in $X$ and in $X_{k}$ of the general fibers the conic bundle $\psi_{\mid U_{1}}$.

Fix $j \in\{1, \ldots, c-1\}$, and recall from 2.7(1) that $\operatorname{Exc}\left(\sigma_{i_{j}}\right) \cap A_{i_{j}}=\varnothing$, so that the birational map $X \rightarrow X_{i_{j}}$ is an isomorphism over $\operatorname{Exc}\left(\sigma_{i_{j}}\right)$. In $X_{i_{j}+1}$ we have

$$
A_{i_{j}+1}=\sigma_{i_{j}}\left(\operatorname{Exc}\left(\sigma_{i_{j}}\right) \cup A_{i_{j}}\right),
$$

hence $\sigma_{i_{j}}\left(\operatorname{Exc}\left(\sigma_{i_{j}}\right)\right)$ is a connected component of $A_{i_{j}+1}$.

Let $x \in \sigma_{i_{j}}\left(\operatorname{Exc}\left(\sigma_{i_{j}}\right)\right) \subset X_{i_{j}+1}$ and let $l \subseteq E_{j} \subset X$ be the transform of the fiber of $\sigma_{i_{j}}$ over $x$.

Let $B_{0} \subseteq H$ be a general irreducible curve which intersects $\pi\left(e^{-1}(l)\right)$. Since $\pi$ is equidimensional and the general fiber of $\pi$ over $B_{0}$ is $\mathbb{P}^{1}$, the inverse image $\pi^{-1}\left(B_{0}\right) \subseteq \mathscr{C}$ is irreducible. Set $S:=e\left(\pi^{-1}\left(B_{0}\right)\right) \subseteq X$, then $S \cap l \neq \varnothing$ by construction. 
Consider the normalizations $B \rightarrow B_{0}$ and $\mathscr{C}_{B} \rightarrow \pi^{-1}\left(B_{0}\right)$ of $B_{0}$ and $\pi^{-1}\left(B_{0}\right)$ respectively; we have induced morphisms $e_{B}: \mathscr{C}_{B} \rightarrow S$ and $\pi_{B}: \mathscr{C}_{B} \rightarrow B$.

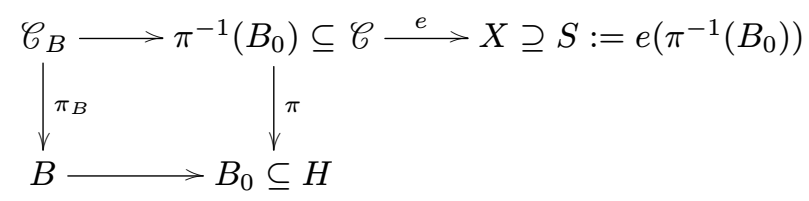

Because $B_{0}$ is general, $B_{0} \cap \operatorname{dom}\left(\iota^{-1}\right) \neq \varnothing$, and $\iota^{-1}$ induces a morphism $\eta: B \rightarrow Y$. Set $B_{1}:=\eta(B) \subset Y$.

Again, since $\varphi$ is equidimensional and the general fiber of $\varphi$ over $B_{1}$ is $\mathbb{P}^{1}$, the inverse image $\varphi^{-1}\left(B_{1}\right) \subset X_{k}$ is irreducible; call $S_{k}$ this surface, which is just the transform of $S \subset X$ under $\sigma$.

Recall that $\varphi$ is finite on $D_{k}$ by (1), and $A_{k} \subset D_{k}$ by 2.6(4), hence no component of a fiber of $\varphi$ can be contained in $A_{k}$. On the other hand, by the generality of $B_{0}$, the general fiber of $\varphi_{\mid S_{k}}$ does not intersect $A_{k}$. Therefore $S_{k}$ can intersect $A_{k}$ at most in a finite number of points.

Consider now $\sigma_{S}:=\sigma_{\mid S}: S \rightarrow S_{k}$. Then $\sigma_{S}$ is an isomorphism over $S_{k} \backslash\left(S_{k} \cap A_{k}\right)$ and $\operatorname{dim}\left(S_{k} \cap A_{k}\right) \leq 0$, hence by Zariski's main theorem $\xi:=\sigma_{S} \circ e_{B}: \mathscr{C}_{B} \rightarrow S_{k}$ is a morphism.

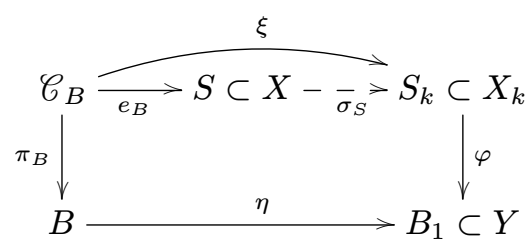

Let $y \in B$ be such that $C:=e_{B}\left(\pi_{B}^{-1}(y)\right) \subset S$ intersects $l$; in particular $C \cap E_{j} \neq \varnothing$, because $l \subseteq E_{j}$. Since $C$ is numerically equivalent in $X$ to a general fiber of $\psi$, we have $-K_{X} \cdot C=2$ and $E_{j} \cdot C=0$; in particular $C$ has at most two irreducible components, because $-K_{X}$ is ample.

Set $r:=\varphi^{-1}(\eta(y))$. Since $r$ is numerically equivalent in $X_{k}$ to a general fiber of $\varphi$, we have $-K_{X_{k}} \cdot r=2$. Recall that no irreducible component of $r$ can be contained in $A_{k}$; on the other hand, $r$ must intersect $A_{k}$, otherwise $\sigma_{S}$ would be an isomorphism over $r, C=\sigma_{S}^{-1}(r)$, and $C \cap E_{j}=\varnothing$, a contradiction.

Let us show that $r$ is an integral fiber of $\varphi$. Indeed let $C_{1}$ be an irreducible component of $r$. If $C_{1} \cap A_{k}=\varnothing$, then $C_{1}$ is contained in the smooth locus of $X_{k}$ and $-K_{X_{k}} \cdot C_{1} \geq 1$. If instead $C_{1} \cap A_{k} \neq \varnothing$, then [11, Lemma 3.8] gives $-K_{X_{k}} \cdot C_{1}>1$. Since $-K_{X_{k}} \cdot r=2$ and $r$ must intersect $A_{k}$, it must be irreducible and reduced.

For every $i \in\{0, \ldots, k-1\}$ let $\widetilde{r}_{i} \subset X_{i}$ be the transform of $r \subset X_{k}$ (where $X_{0}=X$ ). Again by [11, Lemma 3.8] we get $-K_{X} \cdot \widetilde{r}_{0}<-K_{X_{k}} \cdot r=2$, hence $-K_{X} \cdot \widetilde{r}_{0}=1$.

Notice that $\xi\left(\pi_{B}^{-1}(y)\right) \subset S_{k}$ is contained in $r$; on the other hand $\xi$ cannot contract to a point a fiber of $\pi_{B}$, hence $\xi\left(\pi_{B}^{-1}(y)\right)=r$. Then $\widetilde{r}_{0} \subseteq C$, because $C=e_{B}\left(\pi_{B}^{-1}(y)\right)$, and we get $C=\widetilde{r}_{0} \cup C^{\prime}$, where $C^{\prime} \subset X$ is an irreducible curve (and possibly $C^{\prime}=\widetilde{r}_{0}$ if $C$ is non-reduced). 
Since $r \not \subset A_{k}$, we have $\widetilde{r}_{0} \not \subset E_{j}$; in particular $E_{j} \cdot \widetilde{r}_{0} \geq 0$. If $E_{j} \cdot \widetilde{r}_{0}=0$, then also $E_{j} \cdot C^{\prime}=0$ and $C \subset E_{j}$, which is impossible. Hence $E_{j} \cdot \widetilde{r}_{0}>0$, and since $E_{j} \cdot C=0$, we have $E_{j} \cdot C^{\prime}<0$ and $C^{\prime} \neq \widetilde{r}_{0}$.

Consider now the blow-up $\sigma_{i_{j}}: X_{i_{j}} \rightarrow X_{i_{j}+1}$. We have $\operatorname{Exc}\left(\sigma_{i_{j}}\right) \cdot \widetilde{r}_{i_{j}}=E_{j} \cdot \widetilde{r}_{0} \geq 1$, hence using the projection formula we get $-K_{X_{i_{j}+1}} \cdot \widetilde{r}_{i_{j}+1} \geq-K_{X_{i_{j}}} \cdot \widetilde{r}_{i_{j}}+1$. On the other hand [11, Lemma 3.8] gives

$$
1=-K_{X} \cdot \widetilde{r}_{0} \leq-K_{X_{i_{j}}} \cdot \widetilde{r}_{i_{j}} \quad \text { and } \quad-K_{X_{i_{j}+1}} \cdot \widetilde{r}_{i_{j}+1} \leq-K_{X_{k}} \cdot r=2 .
$$

We conclude that $\operatorname{Exc}\left(\sigma_{i_{j}}\right) \cdot \widetilde{r}_{i_{j}}=1,-K_{X} \cdot \widetilde{r}_{0}=-K_{X_{i_{j}}} \cdot \widetilde{r}_{i_{j}}$, and $-K_{X_{i_{j}+1}} \cdot \widetilde{r}_{i_{j}+1}=-K_{X_{k}} \cdot r$, and again by [11, Lemma 3.8] this implies that:

$$
\text { for every } m \in\{0, \ldots, k-1\}, m \neq i_{j} \text {, } \operatorname{Locus}\left(Q_{m}\right) \text { is disjoint from } \widetilde{r}_{m} \text {. }
$$

We show that $C^{\prime}=l$ (recall that $l \subset X$ is the transform of $\sigma_{i_{j}}^{-1}(x) \subset X_{i_{j}}$ ). Since $C^{\prime}$ intersects $\widetilde{r}_{0}$ (because $C=\widetilde{r}_{0} \cup C^{\prime}$ is connected), and $\widetilde{r}_{0} \cap \operatorname{Locus}\left(Q_{0}\right)=\varnothing$ by (2.9), we see that $C^{\prime}$ is not contained in $\operatorname{Locus}\left(Q_{0}\right)$. Iterating this reasoning for every $\sigma_{m}$ with $m \in\left\{0, \ldots, i_{j}-1\right\}$, we see that $C^{\prime}$ intersects the open subset where the birational map $X \rightarrow X_{i_{j}}$ is an isomorphism; let $\widetilde{C}^{\prime} \subset X_{i_{j}}$ be its transform.

If $\sigma_{i_{j}}\left(\widetilde{C}^{\prime}\right)$ were a curve, then by the same reasoning it could not be contained in $\operatorname{Locus}\left(Q_{m}\right)$ for any $m=i_{j}+1, \ldots, k-1$, and in the end we would get a curve $\widetilde{C}_{k}^{\prime} \subset X_{k}$, distinct from $r$, which should belong to $\xi\left(\pi_{B}^{-1}(y)\right)$, which is impossible. Thus $\widetilde{C}^{\prime}$ must be a fiber of $\sigma_{i_{j}}$. On the other hand $\operatorname{Exc}\left(\sigma_{i_{j}}\right) \cdot \widetilde{r}_{i_{j}}=1$, thus $\widetilde{r}_{i_{j}}$ intersects a unique fiber of $\sigma_{i_{j}}$, and $C^{\prime}=l$.

In particular this yields that $x \in \widetilde{r}_{i_{j}+1} \cap \sigma_{i_{j}}\left(\operatorname{Exc}\left(\sigma_{i_{j}}\right)\right)$. Since $x \in \sigma_{i_{j}}\left(\operatorname{Exc}\left(\sigma_{i_{j}}\right)\right)$ was arbitrary, (2.9) implies statement (2).

Let $T_{j} \subset X_{k}$ be the image of $\sigma_{i_{j}}\left(\operatorname{Exc}\left(\sigma_{i_{j}}\right)\right) \subset X_{i_{j}+1}$. By (2) the birational map $X_{i_{j}+1} \rightarrow X_{k}$ yields an isomorphism between $\sigma_{i_{j}}\left(\operatorname{Exc}\left(\sigma_{i_{j}}\right)\right)$ and $T_{j}$, hence $T_{j}$ is smooth of dimension $n-2$, and is contained in the smooth locus of $X_{k}$. Since $\sigma_{i_{j}}\left(\operatorname{Exc}\left(\sigma_{i_{j}}\right)\right)$ is a connected component of $A_{i_{j}+1}$, we deduce that $T_{j}$ is a connected component of $A_{k}$, and $A_{k} \backslash T_{j}$ is closed in $X_{k}$.

By (2.9) the birational map $X_{i_{j}+1} \rightarrow X_{k}$ yields also an isomorphism between $\widetilde{r}_{i_{j}+1}$ and $r$, and $r \cap\left(A_{k} \backslash T_{j}\right)=\varnothing$.

Consider the point $x^{\prime} \in T_{j}$ corresponding to $x \in \sigma_{i_{j}}\left(\operatorname{Exc}\left(\sigma_{i_{j}}\right)\right)$. Then $x^{\prime} \in r \cap T_{j}$ because $x \in \widetilde{r}_{i_{j}+1}$, i.e., $r$ is the fiber of $\varphi$ through $x^{\prime} \in T_{j}$. Again since $x$ was arbitrary in $\sigma_{i_{j}}\left(\operatorname{Exc}\left(\sigma_{i_{j}}\right)\right)$, from $r \cap\left(A_{k} \backslash T_{j}\right)=\varnothing$ we deduce that $\varphi^{-1}\left(\varphi\left(T_{j}\right)\right) \cap\left(A_{k} \backslash T_{j}\right)=\varnothing$, and hence that $\varphi\left(T_{j}\right) \cap \varphi\left(A_{k} \backslash T_{j}\right)=\varnothing$ in $Y$.

Summing up, we have shown that $T_{1}, \ldots, T_{c-1}$ are connected components of $A_{k}$ (so that $A_{k} \backslash\left(T_{1} \cup \cdots \cup T_{c-1}\right)$ is closed in $\left.X_{k}\right)$, and the images $\varphi\left(T_{1}\right), \ldots, \varphi\left(T_{c-1}\right)$, $\varphi\left(A_{k} \backslash\left(T_{1} \cup \cdots \cup T_{c-1}\right)\right)$ are pairwise disjoint in $Y$.

Now set

$$
V:=Y \backslash \varphi\left(A_{k} \backslash\left(T_{1} \cup \cdots \cup T_{c-1}\right)\right) .
$$

Then $V$ is open in $Y, \varphi^{-1}(V) \subseteq \sigma(\operatorname{dom}(\sigma))$, and $T_{1} \cup \cdots \cup T_{c-1} \subset \varphi^{-1}(V)$. Set $U:=\sigma^{-1}\left(\varphi^{-1}(V)\right) \subseteq X$. By definition, $\varphi^{-1}(V) \cap\left(A_{k} \backslash\left(T_{1} \cup \cdots \cup T_{c-1}\right)\right)=\varnothing$; this means that for every $m \in\{0, \ldots, k-1\} \backslash\left\{i_{1}, \ldots, i_{c-1}\right\}$, $\operatorname{Locus}\left(Q_{m}\right)$ is disjoint from the image of $U$ in $X_{m}$. 
We have $E_{1}, \ldots, E_{c-1} \subset U$, because $E_{j}=\sigma^{-1}\left(T_{j}\right)$, and $\psi: U \rightarrow V$ is regular and proper. More precisely, every fiber of $\psi$ over $V$ is one-dimensional, and as before [3, Theorem 4.1(2)] shows that this is a conic bundle and that $V$ is smooth. We have a factorization

$$
U \underset{\sigma_{\mid U}}{\stackrel{\psi}{\rightarrow} \varphi^{-1}(V) \underset{\varphi}{\longrightarrow} V} V
$$

and $\sigma_{\mid U}$ is just the blow-up of $T_{1} \cup \cdots \cup T_{c-1}$, so we get (3). For every $j \in\{1, \ldots, c-1\}$ we have $Z_{j}=\psi\left(E_{j}\right)=\varphi\left(T_{j}\right)$, so $Z_{1}, \ldots, Z_{c-1}$ are pairwise disjoint. Now let $\widehat{E}_{j} \subset U$ be the transform of $\varphi^{-1}\left(Z_{j}\right)$. Then $\psi^{-1}\left(Z_{j}\right)=E_{j} \cup \widehat{E}_{j}$, and the rest of statement (4) follows from standard arguments on conic bundles. Just notice that if for some $j \in\{1, \ldots, c-1\}$ we have $\left[\widehat{f}_{j}\right] \in \mathcal{N}_{1}\left(E_{j}, X\right)$, then $\left[\sigma\left(\widehat{f}_{j}\right)\right] \in \mathcal{N}_{1}\left(T_{j}, X_{k}\right) \subseteq \mathcal{N}_{1}\left(A_{k}, X_{k}\right) \subseteq \mathcal{N}_{1}\left(D_{k}, X_{k}\right)$, which is impossible because $\sigma\left(\widehat{f}_{j}\right)$ is a fiber of $\varphi$ and $\mathrm{NE}(\varphi) \not \subset \mathcal{N}_{1}\left(D_{k}, X_{k}\right)$ by assumption.

Proof of Theorem 1.2. - If $\mathcal{N}_{1}(D, X)=\mathcal{N}_{1}(X)$ for every prime divisor $D \subset X$, then we have (ii) (just notice that if $D_{1}, D_{2} \subset X$ are two disjoint divisors, then $\mathcal{N}_{1}\left(D_{1}, X\right) \subseteq D_{2}^{\perp} \subsetneq \mathcal{N}_{1}(X)$, see Remark 3.1.2).

Suppose now that there exists a prime divisor $D \subset X$ with $\operatorname{codim} \mathcal{N}_{1}(D, X)>0$, and consider a special Mori program for $-D$ (which exists by Proposition 2.4). Let $E_{1}, \ldots, E_{s} \subset X$ be the $\mathbb{P}^{1}$-bundles determined by the Mori program.

If $s \geq 1$, by 2.7(3) we have $-K_{X} \cdot f_{1}=1$, where $f_{1} \subset E_{1}$ is a fiber of the $\mathbb{P}^{1}$-bundle; this is impossible because $\iota_{X}>1$.

Therefore $s=0$, and 2.7(2) yields that $\operatorname{codim} \mathcal{N}_{1}(D, X)=1$ and $Q_{k} \not \subset \mathcal{N}_{1}\left(D_{k}, X_{k}\right)$, so that Lemma 2.8 applies.

We show that $k=0$ and $X=X_{k}$. Indeed if not, we have $A_{k} \neq \varnothing$ in $X_{k}$ (see 2.6(4)). Take $r$ a fiber of $\varphi$ intersecting $A_{k}$. Then, using [11, Lemma 3.8] as in the proof of Lemma 2.8, we see that $r$ is integral, and that the transform $\widetilde{r} \subset X$ of $r$ has anticanonical degree 1 in $X$, a contradiction.

Thus $X=X_{k}$ and we get a conic bundle $\varphi: X \rightarrow Y$, which is finite on $D$. Since $X$ contains no curves of anticanonical degree $1, \varphi$ must be a smooth fibration in $\mathbb{P}^{1}$. Then $Y$ is Fano by [21, Proposition 4.3], and finally we have $\iota_{Y} \geq \iota_{X}=2$ by [7, Lemme 2.5].

\section{Divisors with minimal Picard number}

Let $X$ be a Fano manifold, and consider

$$
c_{X}:=\max \left\{\operatorname{codim} \mathcal{N}_{1}(D, X) \mid D \text { is a prime divisor in } X\right\} .
$$

We always have $0 \leq c_{X} \leq \rho_{X}-1$. If $S$ is a Del Pezzo surface, then $c_{S}=\rho_{S}-1 \in\{0, \ldots, 8\}$.

Example 3.1. - Consider a Fano manifold $X=S \times T$, where $S$ is a Del Pezzo surface. Then $c_{X}=\max \left\{\rho_{S}-1, c_{T}\right\}$. More precisely, for any prime divisor $D \subset X$, we have three possibilities:

- $D=C \times T$ where $C \subset S$ is a curve, and $\operatorname{codim} \mathcal{N}_{1}(D, X)=\rho_{S}-1$;

- $D=S \times D_{T}$ where $D_{T} \subset T$ is a divisor, and codim $\mathcal{N}_{1}(D, X)=\operatorname{codim} \mathcal{N}_{1}\left(D_{T}, T\right) \leq c_{T}$;

- $D$ dominates both $S$ and $T$ under the projections, and codim $\mathcal{N}_{1}(D, X) \leq \rho_{S}-1$. 
Indeed suppose that $D \subset X$ is a prime divisor with $\operatorname{codim} \mathcal{N}_{1}(D, X)>\rho_{S}-1$. Then $\operatorname{dim} \mathcal{N}_{1}(D, X)<\rho_{T}+1$, so that $D$ cannot dominate $T$ under the projection, and $D=S \times D_{T}$.

Example 3.2. - If $X$ is a Fano manifold with pseudo-index $\iota_{X} \geq 3$ (for instance $X=\mathbb{P}^{n_{1}} \times \cdots \times \mathbb{P}^{n_{r}}$ with $n_{i} \geq 2$ for all $\left.i=1, \ldots, r\right)$, then $c_{X}=0$ by Theorem 1.2.

We are going to use the results of Section 2.2 to prove the following.

Theorem 3.3. - For any Fano manifold $X$ we have $c_{X} \leq 8$. Moreover:

- if $c_{X} \geq 4$ then $X \cong S \times T$ where $S$ is a Del Pezzo surface, $\rho_{S}=c_{X}+1$, and $c_{T} \leq c_{X}$;

- if $c_{X}=3$ then there exists a flat, quasi-elementary contraction $X \rightarrow T$ where $T$ is an $(n-2)$-dimensional Fano manifold, $\rho_{X}-\rho_{T}=4$, and $c_{T} \leq 3$.

A contraction $\varphi$ is quasi-elementary if $\operatorname{ker} \varphi_{*}$ is generated by the numerical classes of the curves contained in a general fiber of $\varphi$; we refer the reader to [10] for properties of quasielementary contractions. In particular, in the case where $c_{X}=3$ in Theorem 3.3, the general fiber of the contraction $X \rightarrow T$ is a Del Pezzo surface $S$ with $\rho_{S} \geq 4$.

Example 3.4 (Codimension 3). - Let $n \geq 3$ and $Z=\mathbb{P}_{\mathbb{P}^{n-2}}\left(\theta^{\oplus 2} \oplus \theta(1)\right)$. Then $Z$ is a toric Fano manifold with $\rho_{Z}=2$, and the $\mathbb{P}^{2}$-bundle $Z \rightarrow \mathbb{P}^{n-2}$ has three pairwise disjoint sections $T_{1}, T_{2}, T_{3} \subset Z$ which are closed under the torus action. Let $X \rightarrow Z$ be the blow-up of $T_{1}, T_{2}, T_{3}$. Then $X$ is Fano with $\rho_{X}=5$, and it has a smooth morphism $X \rightarrow \mathbb{P}^{n-2}$ such that every fiber is the Del Pezzo surface $S$ with $\rho_{S}=4$. If $E \subset X$ is one of the exceptional divisors of the blow-up, one easily checks that $\rho_{X}-\rho_{E}=\operatorname{codim} \mathcal{N}_{1}(E, X)=3$, hence $c_{X} \geq 3$. However $X$ is not a product, thus $c_{X}=3$ by Theorem 3.3.

3.5. - The proof of Theorem 3.3 will take all the rest of Section 3; we will proceed in several steps. Section 3.1 gathers some preliminary remarks and lemmas. In Section 3.2 we treat the case $c_{X} \geq 4$, and we show that $X \cong S \times T$, where $S$ is a Del Pezzo surface with $\rho_{S}=c_{X}+1$, and $T$ a Fano manifold with $c_{T} \leq c_{X}$ (see Proposition 3.2.1, and 3.2.3 for an outline of its proof). In particular this implies that $c_{X} \leq 8$, because $\rho_{S} \leq 9$.

The case $c_{X}=3$ is more delicate, as we have to treat separately the two following cases:

(3.6.a) for every prime divisor $D \subset X$ with $\operatorname{codim} \mathcal{N}_{1}(D, X)=3$, and for every special Mori program for $-D$, we have $\mathcal{N}_{1}\left(D_{k}, X_{k}\right)=\mathcal{N}_{1}\left(X_{k}\right)$ (notation as in Lemma 2.6);

(3.6.b) there exist a prime divisor $D \subset X$ with $\operatorname{codim} \mathcal{N}_{1}(D, X)=3$, and a special Mori program for $-D$, such that $\mathcal{N}_{1}\left(D_{k}, X_{k}\right) \subsetneq \mathcal{N}_{1}\left(X_{k}\right)$.

The first case (3.6.a) is treated together with the case $c_{X} \geq 4$, in Section 3.2. In the end we reach a contradiction, hence a posteriori we conclude that (3.6.a) never happens (see Corollary 3.2.2). The second case (3.6.b) is treated in Section 3.3, where we show the existence of a flat, quasi-elementary contraction $X \rightarrow T$, where $T$ is an $(n-2)$-dimensional Fano manifold, $\rho_{X}-\rho_{T}=4$, and $c_{T} \leq 3$ (see Proposition 3.3.1, and 3.3.3 for an outline of its proof). 


\subsection{Preliminary results}

In this section we collect some remarks and lemmas which will be used in the proof of Theorem 3.3.

REMARK 3.1.1. - Let $X$ be a projective manifold, $\varphi: X \rightarrow Y$ a contraction such that $-K_{X}$ is $\varphi$-ample and $\operatorname{dim} Y>0$, and $D$ a divisor in $X$ such that $\operatorname{ker} \varphi_{*} \subseteq D^{\perp}$. Then we have the following:

(1) $\operatorname{dim} Y=1+\operatorname{dim} \varphi(\operatorname{Supp} D)$ and $D=\varphi^{*}\left(D_{Y}\right), D_{Y}$ a Cartier divisor in $Y$;

(2) if $D$ is a prime divisor, then $\varphi(D)$ is a prime Cartier divisor, and $D=\varphi^{*}(\varphi(D))$;

(3) if $D$ is a smooth prime divisor, let $\varphi(D)^{\nu} \rightarrow \varphi(D)$ be the normalization. Then the morphism $\varphi_{D}: D \rightarrow \varphi(D)^{\nu}$ induced by $\varphi_{\mid D}$ is a contraction, and $-K_{D}$ is $\varphi_{D}$-ample;

(4) if $D$ is a smooth prime divisor and $Y$ is smooth, then $\varphi(D)$ is a smooth prime divisor.

Proof. - By [16, Theorem 3.7(4)] there exists a Cartier divisor $D_{Y}$ on $Y$ such that $D=\varphi^{*}\left(D_{Y}\right)$. Then $\operatorname{Supp} D_{Y}=\varphi(\operatorname{Supp} D)$, so we have (1).

If $D$ is a prime divisor, then $D_{Y}$ is a prime divisor supported on $\varphi(D)$, namely $D_{Y}=\varphi(D)$, and we have (2).

For (3), $\varphi_{D}$ is surjective with connected fibers onto a normal projective variety, hence a contraction. Let $i: D \hookrightarrow X$ be the inclusion and take $\gamma \in \overline{\mathrm{NE}}(D) \cap \operatorname{ker}\left(\varphi_{D}\right)_{*}$ with $\gamma \neq 0$. The restriction $\left(-K_{X}\right)_{\mid D}$ is $\varphi_{D}$-ample, hence $\left(-K_{X}\right)_{\mid D} \cdot \gamma>0$. Moreover $i_{*}(\gamma) \in \operatorname{ker} \varphi_{*}$, so that

$$
-K_{D} \cdot \gamma=-\left(K_{X}+D\right) \cdot i_{*}(\gamma)=-K_{X} \cdot i_{*}(\gamma)>0
$$

and $-K_{D}$ is $\varphi_{D}$-ample.

For (4), let $y \in \varphi(D)$ and let $f \in \vartheta_{Y, y}$ be a local equation for $\varphi(D)$. Then $\varphi^{*}(f)$ is a local equation for $D$ near the fiber over $y$. Since $D$ is smooth, the differential $d_{x}\left(\varphi^{*}(f)\right)$ is non-zero, where $x \in \varphi^{-1}(y)$. Then $d_{y} f$ is non-zero, hence $\varphi(D)$ is smooth at $y$.

Remark 3.1.2. - Let $X$ be a projective manifold, $Z \subset X$ a closed subset, and $D \subset X$ a prime divisor. If $Z \cap D=\varnothing$, then $D \cdot C=0$ for every curve $C \subset Z$, hence $\mathcal{N}_{1}(Z, X) \subseteq D^{\perp}$.

ReMARK 3.1.3. - Let $X$ be a projective manifold, $E \subset X$ a smooth prime divisor which is a $\mathbb{P}^{1}$-bundle with fiber $f \subset E$, and $D \subset X$ a prime divisor with $D \cdot f>0$. Then the following holds:

(1) $\operatorname{dim} \mathcal{N}_{1}(D \cap E, X) \geq \operatorname{dim} \mathcal{N}_{1}(E, X)-1$ and $\mathcal{N}_{1}(E, X)=\mathbb{R}[f]+\mathcal{N}_{1}(D \cap E, X)$;

(2) either $[f] \in \mathcal{N}_{1}(D \cap E, X)$ and $\mathcal{N}_{1}(D \cap E, X)=\mathcal{N}_{1}(E, X)$, or $[f] \notin \mathcal{N}_{1}(D \cap E, X)$ and $\mathcal{N}_{1}(D \cap E, X)$ has codimension 1 in $\mathcal{N}_{1}(E, X)$;

(3) for every irreducible curve $C \subset E$ we have $C \equiv \lambda f+\mu C^{\prime}$, where $C^{\prime}$ is an irreducible curve contained in $D \cap E, \lambda, \mu \in \mathbb{R}$, and $\mu \geq 0$.

Proof. - Let $\pi: E \rightarrow F$ be the $\mathbb{P}^{1}$-bundle structure on $E$, and consider the push-forward $\pi_{*}: \mathcal{N}_{1}(E) \rightarrow \mathcal{N}_{1}(F)$. This is a surjective linear map with kernel $\mathbb{R}[f]_{E}$.

Since $D \cdot f>0$, we have $\pi(D \cap E)=F$, thus $\pi_{*}\left(\mathcal{N}_{1}(D \cap E, E)\right)=\mathcal{N}_{1}(F)$. Therefore $\mathcal{N}_{1}(E)=\mathbb{R}[f]_{E}+\mathcal{N}_{1}(D \cap E, E)$, and applying $i_{*}$ (where $i: E \hookrightarrow X$ is the inclusion) we get (1) and (2). Statement (3) follows from [18, Lemma 3.2 and Remark 3.3]. 
Remark 3.1.4. - Let $X$ be a Fano manifold and $D, E \subset X$ prime divisors with

$$
\mathcal{N}_{1}(D \cap E, X) \subseteq E^{\perp} .
$$

Suppose that $E$ is a smooth $\mathbb{P}^{1}$-bundle with fiber $f \subset E$, such that $E \cdot f=-1$ and $D \cdot f>0$.

Then the half-line $\mathbb{R}_{\geq 0}[f] \subset \mathrm{NE}(X)$ is an extremal ray of type $(n-1, n-2)^{s m}$, with contraction $\varphi: X \rightarrow Y$ where $E=\operatorname{Exc}(\varphi)$ and $Y$ is Fano.

Proof. - Notice first of all that $\left(-K_{X}+E\right) \cdot f=0$.

Let $C \subset X$ be an irreducible curve. If $C \not \subset E$, then $\left(-K_{X}+E\right) \cdot C>0$. If $C \subseteq D \cap E$, then $E \cdot C=0$, and again $\left(-K_{X}+E\right) \cdot C>0$.

Assume now that $C \subseteq E$. By 3.1.3(3) we have $C \equiv \lambda f+\mu C^{\prime}$, where $C^{\prime}$ is a curve contained in $D \cap E, \lambda, \mu \in \mathbb{R}$, and $\mu \geq 0$. Thus

$$
\left(-K_{X}+E\right) \cdot C=\mu\left(-K_{X}+E\right) \cdot C^{\prime} \geq 0
$$

and $\left(-K_{X}+E\right) \cdot C=0$ if and only if $\mu=0$, if and only if $[C] \in \mathbb{R}_{\geq 0}[f]$. Therefore $-K_{X}+E$ is nef, and $\left(-K_{X}+E\right)^{\perp} \cap \mathrm{NE}(X)=\mathbb{R}_{\geq 0}[f]$ is an extremal ray.

Let $\varphi: X \rightarrow Y$ be the contraction of $\mathbb{R}_{\geq 0}[f]$; clearly $\operatorname{Exc}(\varphi)=E$. Since $\left(-K_{X}+E\right) \cdot C>0$ for every curve $C \subset D \cap E, \varphi$ is finite on $D \cap E$. Thus if $F \subset E$ is a fiber of $\varphi$, then $F \cap D \neq \varnothing$ (because $D \cdot \operatorname{NE}(\varphi)>0$ ), and $\operatorname{dim}(F \cap D)=0$. This yields that $\operatorname{dim} F=1$, and by [1, Theorem 2.3] $\mathbb{R}_{\geq 0}[f]$ is of type $(n-1, n-2)^{s m}$ and $Y$ is smooth.

Finally $-K_{X}+E=\varphi^{*}\left(-K_{Y}\right)$, thus $-K_{Y}$ is ample and $Y$ is Fano (notice that $\mathrm{NE}(Y)$ is closed, because $\left.\mathrm{NE}(Y)=\varphi_{*}(\mathrm{NE}(X))\right)$.

Lemma 3.1.5. - Let $X$ be a Fano manifold and $D, E \subset X$ prime divisors with

$$
\mathcal{N}_{1}(D \cap E, X)=\mathcal{N}_{1}(E, X) \cap D^{\perp} \subseteq E^{\perp} .
$$

Suppose that $E$ is a smooth $\mathbb{P}^{1}$-bundle with fiber $f \subset E$, such that $E \cdot f=-1$ and $D \cdot f>0$.

Then $E \cong \mathbb{P}^{1} \times F$ where $F$ is a Fano manifold, and $D \cap E=\{p t s\} \times F$. Moreover the half-line $\mathbb{R}_{\geq 0}[f]$ is an extremal ray of type $(n-1, n-2)^{s m}$, it is the unique extremal ray having negative intersection with $E$, and the target of its contraction is Fano.

Proof. - Consider the divisor $D_{\mid E}$ in $E$. We have $\operatorname{Supp}\left(D_{\mid E}\right)=D \cap E$, and if $C \subseteq D \cap E$ is an irreducible curve, then $[C] \in \mathcal{N}_{1}(D \cap E, X) \subseteq D^{\perp}$, so that $D_{\mid E} \cdot C=D \cdot C=0$. Therefore $D_{\mid E}$ is nef.

Let $i: E \hookrightarrow X$ be the inclusion and take $\gamma \in \overline{\mathrm{NE}}(E) \cap\left(D_{\mid E}\right)^{\perp}$ with $\gamma \neq 0$. Then $i_{*}(\gamma) \in \mathcal{N}_{1}(E, X) \cap D^{\perp} \subseteq E^{\perp}$, hence:

$$
-K_{E} \cdot \gamma=-\left(K_{X}+E\right) \cdot i_{*}(\gamma)=-K_{X} \cdot i_{*}(\gamma)=\left(-K_{X}\right)_{\mid E} \cdot \gamma>0 .
$$

By the contraction theorem, there exists a contraction $g: E \rightarrow Z$ such that $-K_{E}$ is $g$-ample and $\mathrm{NE}(g)=\overline{\mathrm{NE}}(E) \cap\left(D_{\mid E}\right)^{\perp}$ (see [16, Theorem 3.7(3)]). Notice that $D_{\mid E} \cdot f=D \cdot f>0$, hence $g$ does not contract the fibers of the $\mathbb{P}^{1}$-bundle on $E$, and $\operatorname{dim} Z \geq 1$. On the other hand $g$ sends $D \cap E$ to a union of points, so that $\operatorname{dim} Z=1$ by 3.1.1(1). More precisely, since $g(f)=Z$, we get $Z \cong \mathbb{P}^{1}$. The general fiber $F$ of $g$ is a Fano manifold of dimension $n-2$, because $-K_{E}$ is $g$-ample.

By [11, Lemma 4.9] we conclude that $E \cong \mathbb{P}^{1} \times F$ and $g$ is the projection onto $\mathbb{P}^{1}$. Since $D \cdot f>0, D \cap E$ dominates $F$ under the projection, and is sent by $g$ to a union of points; therefore $D \cap E=\{p t s\} \times F$. 
Using Remark 3.1.4, we see that $\mathbb{R}_{\geq 0}[f]$ is an extremal ray of type $(n-1, n-2)^{s m}$, and the target of its contraction is Fano.

Finally let $R$ be an extremal ray of $X$ with $E \cdot R<0$. Then $R \subseteq \mathrm{NE}(E, X) \subseteq \mathrm{NE}(X)$, thus $R$ must be a one-dimensional face of $\operatorname{NE}(E, X)$. ${ }^{(2)}$ Since $E \cong \mathbb{P}^{1} \times F$, we have $\mathrm{NE}(E)=\mathbb{R}_{\geq 0}[f]_{E}+\mathrm{NE}(\{p t\} \times F, E)$ and $\mathrm{NE}(E, X)=\mathbb{R}_{\geq 0}[f]+\mathrm{NE}(\{p t\} \times F, X)$. On the other hand $\operatorname{NE}(\{p t\} \times F, X) \subset \mathcal{N}_{1}(\{p t\} \times F, X)=\mathcal{N}_{1}(D \cap E, X) \subseteq E^{\perp}$, therefore $R=\mathbb{R}_{\geq 0}[f]$.

REMARK 3.1.6. - Let $X$ be a projective manifold and $E_{0} \subset X$ a smooth prime divisor which is a $\mathbb{P}^{1}$-bundle with fiber $f_{0} \subset E_{0}$. Let $E_{1}, \ldots, E_{s} \subset X$ be pairwise disjoint prime divisors such that $E_{0} \neq E_{i}$ and $E_{0} \cap E_{i} \neq \varnothing$ for every $i=1, \ldots, s$. Then either $E_{1} \cdot f_{0}=\cdots=E_{s} \cdot f_{0}=0$, or $E_{i} \cdot f_{0}>0$ for $i=1, \ldots, s$.

Proof. - For every $i=1, \ldots, s$ we have $E_{i} \cdot f_{0} \geq 0$, because $E_{0} \neq E_{i}$.

Suppose that there exists $j \in\{1, \ldots, s\}$ such that $E_{j} \cdot f_{0}=0$. Since $E_{0} \cap E_{j} \neq \varnothing$, this implies that $E_{j}$ contains a fiber $\bar{f}_{0}$ of the $\mathbb{P}^{1}$-bundle structure on $E_{0}$. If $i \in\{1, \ldots, s\}, i \neq j$, we have $E_{i} \cap E_{j}=\varnothing$, in particular $E_{i} \cap \bar{f}_{0}=\varnothing$ and hence $E_{i} \cdot f_{0}=0$.

Lemma 3.1.7. - Let $X$ be a Fano manifold and $D \subset X$ a prime divisor with $\operatorname{codim} \mathcal{N}_{1}(D, X)=c_{X}$. Let $E_{1}, \ldots, E_{s} \subset X$ be pairwise disjoint prime divisors such that:

$D \cap E_{i} \neq \varnothing, \quad D \neq E_{i}$, and $\operatorname{codim} \mathcal{N}_{1}\left(D \cap E_{i}, X\right) \leq c_{X}+1$, for every $i=1, \ldots, s$.

If $s \geq 2$, then $\operatorname{codim} \mathcal{N}_{1}\left(D \cap E_{i}, X\right)=c_{X}+1$ for every $i=1, \ldots, s$, and

$$
\mathcal{N}_{1}\left(D \cap E_{i}, X\right)=\mathcal{N}_{1}(D, X) \cap E_{j}^{\perp} \text { for every } i \neq j .
$$

If $s \geq 3$, then there exists a linear subspace $L \subset \mathcal{N}_{1}(X)$, of codimension $c_{X}+1$, such that $L=\mathcal{N}_{1}\left(D \cap E_{i}, X\right)=\mathcal{N}_{1}(D, X) \cap E_{i}^{\perp}$ for every $i=1, \ldots, s$.

Proof. - Assume that $s \geq 2$, and let $i, j \in\{1, \ldots, s\}$ with $i \neq j$. Since $E_{i} \cap E_{j}=\varnothing$, we have $\mathcal{N}_{1}\left(D \cap E_{i}, X\right) \subseteq E_{j}^{\perp}$ by Remark 3.1.2. On the other hand, since $D \cap E_{j} \neq \varnothing$ and $D \neq E_{j}$, there exists some curve $C \subset D$ with $E_{j} \cdot C>0$, so that $\mathcal{N}_{1}(D, X) \nsubseteq E_{j}^{\perp}$. Therefore we get:

$$
\mathcal{N}_{1}\left(D \cap E_{i}, X\right) \subseteq \mathcal{N}_{1}(D, X) \cap E_{j}^{\perp} \subsetneq \mathcal{N}_{1}(D, X),
$$

hence $\rho_{X}-c_{X}-1 \leq \operatorname{dim} \mathcal{N}_{1}\left(D \cap E_{i}, X\right) \leq \operatorname{dim} \mathcal{N}_{1}(D, X) \cap E_{j}^{\perp}=\operatorname{dim} \mathcal{N}_{1}(D, X)-1=$ $\rho_{X}-c_{X}-1$, and this yields the statement.

Assume now that $s \geq 3$, and set $L:=\mathcal{N}_{1}\left(D \cap E_{1}, X\right)$; the first part already gives that $\operatorname{codim} L=c_{X}+1$ and that $L=\mathcal{N}_{1}(D, X) \cap E_{i}^{\perp}$ for every $i=2, \ldots, s$. If $i, j \in\{2, \ldots, s\}$ are distinct, again by the first part we get

$$
L=\mathcal{N}_{1}(D, X) \cap E_{i}^{\perp}=\mathcal{N}_{1}\left(D \cap E_{j}, X\right)=\mathcal{N}_{1}(D, X) \cap E_{1}^{\perp} .
$$

(2) Since $F$ and $E$ are Fano, the cones $\mathrm{NE}(F), \mathrm{NE}(E), \mathrm{NE}(E, X)$, etc. are closed and polyhedral. 
Lemma 3.1.8. - Let $X$ be a Fano manifold and $D \subset X$ a prime divisor with $\operatorname{codim} \mathcal{N}_{1}(D, X)=c_{X}$. Let $E_{1}, \ldots, E_{s} \subset X$ be pairwise disjoint smooth prime divisors, and suppose that $E_{i}$ is a $\mathbb{P}^{1}$-bundle with fiber $f_{i} \subset E_{i}$, such that $E_{i} \cdot f_{i}=-1$ and $D \cdot f_{i}>0$, for every $i=1, \ldots, s$.

Assume that $s \geq 2$. Then $\operatorname{codim} \mathcal{N}_{1}\left(E_{i}, X\right)=c_{X}$ and $\operatorname{codim} \mathcal{N}_{1}\left(D \cap E_{i}, X\right)=c_{X}+1$ for every $i=1, \ldots, s$; moreover $\mathcal{N}_{1}\left(D \cap E_{i}, X\right)=\mathcal{N}_{1}(D, X) \cap E_{j}^{\perp}$ for every $i \neq j$.

Proof. - Let $i \in\{1, \ldots, s\}$. We have $D \cap E_{i} \neq \varnothing$ and $D \neq E_{i}$ because $D \cdot f_{i}>0$ and $E_{i} \cdot f_{i}=-1$. Since $D \cdot f_{i}>0$, by 3.1.3(1) and by the definition of $c_{X}$ we have

$$
\operatorname{codim} \mathcal{N}_{1}\left(D \cap E_{i}, X\right) \leq \operatorname{codim} \mathcal{N}_{1}\left(E_{i}, X\right)+1 \leq c_{X}+1
$$

Therefore Lemma 3.1.7 yields that $\mathcal{N}_{1}\left(D \cap E_{i}, X\right)=\mathcal{N}_{1}(D, X) \cap E_{j}^{\perp}$ if $i \neq j$, and $\operatorname{codim} \mathcal{N}_{1}\left(D \cap E_{i}, X\right)=c_{X}+1$. By (3.1.9) we get $\operatorname{codim} \mathcal{N}_{1}\left(E_{i}, X\right)=c_{X}$.

Lemma 3.1.10. - Let $X$ be a Fano manifold and $D \subset X$ a prime divisor with $\operatorname{codim} \mathcal{N}_{1}(D, X)=c_{X}$. Let $E_{1}, \ldots, E_{s}, \widehat{E}_{1}, \ldots, \widehat{E}_{s} \subset X$ be prime divisors such that $E_{i}$ and $\widehat{E}_{i}$ are smooth $\mathbb{P}^{1}$-bundles, with fibers respectively $f_{i} \subset E_{i}$ and $\widehat{f}_{i} \subset \widehat{E}_{i}$, and moreover:

$$
E_{i} \cdot f_{i}=\widehat{E}_{i} \cdot \widehat{f}_{i}=-1, \quad D \cdot f_{i}>0, \quad E_{i} \cdot \widehat{f}_{i}>0, \quad \widehat{E}_{i} \cdot f_{i}>0, \quad\left[\widehat{f}_{i}\right] \notin \mathcal{N}_{1}\left(E_{i}, X\right),
$$

and no fiber $\widehat{f}_{i}$ is contained in $D$, for every $i=1, \ldots, s$. We assume also that $E_{1} \cup \widehat{E}_{1}, \ldots, E_{s} \cup \widehat{E}_{s}$ are pairwise disjoint, and that $s \geq 2$.

Then $\operatorname{codim} \mathcal{N}_{1}\left(E_{i}, X\right)=\operatorname{codim} \mathcal{N}_{1}\left(\widehat{E}_{i}, X\right)=c_{X}$ and $\left[f_{i}\right] \notin \mathcal{N}_{1}\left(\widehat{E}_{i}, X\right)$ for every $i=1, \ldots, s$.

Proof. - Lemma 3.1.8 (applied to $D$ and $\left.E_{1}, \ldots, E_{s}\right)$ shows that codim $\mathcal{N}_{1}\left(E_{i}, X\right)=c_{X}$ for every $i=1, \ldots, s$.

Fix $i \in\{1, \ldots, s\}$. Since $\mathcal{N}_{1}\left(E_{i} \cap \widehat{E}_{i}, X\right) \subseteq \mathcal{N}_{1}\left(E_{i}, X\right)$, we have $\left[\widehat{f}_{i}\right] \notin \mathcal{N}_{1}\left(E_{i} \cap \widehat{E}_{i}, X\right)$. Because $E_{i} \cdot \widehat{f}_{i}>0,3.1 .3(2)$ yields that $\mathcal{N}_{1}\left(E_{i} \cap \widehat{E}_{i}, X\right)$ has codimension 1 in $\mathcal{N}_{1}\left(\widehat{E}_{i}, X\right)$. Recall that by the definition of $c_{X}$ we have $\operatorname{codim} \mathcal{N}_{1}\left(\widehat{E}_{i}, X\right) \leq c_{X}$, so that $\operatorname{codim} \mathcal{N}_{1}\left(E_{i} \cap \widehat{E}_{i}, X\right) \leq c_{X}+1$.

Let us show that

$$
\operatorname{codim} \mathcal{N}_{1}\left(E_{i} \cap \widehat{E}_{i}, X\right)=c_{X}+1 \text { and } \operatorname{codim} \mathcal{N}_{1}\left(\widehat{E}_{i}, X\right)=c_{X} .
$$

If $D \cap \widehat{E}_{i}=\varnothing$, then $\mathcal{N}_{1}\left(E_{i} \cap \widehat{E}_{i}, X\right) \subseteq \mathcal{N}_{1}\left(E_{i}, X\right) \cap D^{\perp}$ (see Remark 3.1.2); on the other hand $\mathcal{N}_{1}\left(E_{i}, X\right) \cap D^{\perp} \subsetneq \mathcal{N}_{1}\left(E_{i}, X\right)$, because $D \cdot f_{i}>0$. This yields codim $\mathcal{N}_{1}\left(E_{i} \cap \widehat{E}_{i}, X\right)=$ $c_{X}+1$.

If instead $D \cap \widehat{E}_{i} \neq \varnothing$, then $D \cdot \widehat{f}_{i}>0$, because $D$ cannot contain any curve $\widehat{f}_{i}$. Thus we can apply Lemma 3.1 .8 to the divisors $D$ and $E_{1}, \ldots, E_{i-1}, \widehat{E}_{i}, E_{i+1}, \ldots, E_{s}$, and we deduce that $\operatorname{codim} \mathcal{N}_{1}\left(\widehat{E}_{i}, X\right)=c_{X}$. Hence we have (3.1.11).

Since $\widehat{E}_{i} \cdot f_{i}>0$ and $\operatorname{codim} \mathcal{N}_{1}\left(E_{i}, X\right)=c_{X}=\operatorname{codim} \mathcal{N}_{1}\left(E_{i} \cap \widehat{E}_{i}, X\right)-1$, again by 3.1.3(2) we get $\left[f_{i}\right] \notin \mathcal{N}_{1}\left(E_{i} \cap \widehat{E}_{i}, X\right)$. For dimensional reasons $\mathcal{N}_{1}\left(E_{i} \cap \widehat{E}_{i}, X\right)=$ $\mathcal{N}_{1}\left(E_{i}, X\right) \cap \mathcal{N}_{1}\left(\widehat{E}_{i}, X\right)$, and we conclude that $\left[f_{i}\right] \notin \mathcal{N}_{1}\left(\widehat{E}_{i}, X\right)$. 


\subsection{The case where $X$ is a product}

The main results of this section are the following.

Proposition 3.2.1. - Let $X$ be a Fano manifold such that either $c_{X} \geq 4$, or $c_{X}=3$ and $X$ satisfies (3.6.a).

Then $X \cong S \times T$, where $S$ is a Del Pezzo surface with $\rho_{S}=c_{X}+1$, and $c_{T} \leq c_{X}$. In particular, $c_{X} \leq 8$.

Corollary 3.2.2. - Let $X$ be a Fano manifold with $c_{X}=3$. Then $X$ satisfies (3.6.b).

Proof of Corollary 3.2.2. - By contradiction, suppose that $X$ satisfies (3.6.a). Then by Proposition 3.2.1 we have $X \cong S \times T$ and $\rho_{S}=4$, i.e., $S$ is the blow-up of $\mathbb{P}^{2}$ in three non-collinear points. Consider the sequence:

$$
X \cong S \times T \longrightarrow S_{1} \times T \longrightarrow \mathbb{F}_{1} \times T \longrightarrow \mathbb{P}^{1} \times T,
$$

where $S_{1}$ is the blow-up of $\mathbb{P}^{2}$ in two distinct points. Let $C \subset \mathbb{F}_{1}$ be the section of the $\mathbb{P}^{1}$-bundle containing the two points blown up under $S \rightarrow \mathbb{F}_{1}$. Let moreover $\widetilde{C} \subset S$ be its transform, and $D:=\widetilde{C} \times T \subset X$. Then $\operatorname{codim} \mathcal{N}_{1}(D, X)=3$, and the sequence above is a special Mori program for $-D$. The image of $D$ in $\mathbb{F}_{1} \times T$ is $C \times T$, and $\mathcal{N}_{1}\left(C \times T, \mathbb{F}_{1} \times T\right) \subsetneq$ $\mathcal{N}_{1}\left(\mathbb{F}_{1} \times T\right)$. Thus we have a contradiction with (3.6.a).

3.2.3. - Outline of the proof of Proposition 3.2.1. There are three preparatory steps, and then the actual proof.

The first step is to apply the construction of Section 2.2 to a prime divisor $D \subset X$ with $\operatorname{codim} \mathcal{N}_{1}(D, X)=c_{X}$. We consider a special Mori program for $-D$, and this determines pairwise disjoint $\mathbb{P}^{1}$-bundles $E_{1}, \ldots, E_{s} \subset X$ as in Lemma 2.7; we denote by $f_{i} \subset E_{i}$ a fiber. The crucial property here is that $s \geq 3$ : indeed $s \geq \operatorname{codim} \mathcal{N}_{1}(D, X)-1=c_{X}-1$, so that $s \geq 3$ if $c_{X} \geq 4$. On the other hand if $c_{X}=3$ we have $s=3$ by (3.6.a). Then for $i=1, \ldots, s$ we show that $\operatorname{codim} \mathcal{N}_{1}\left(E_{i}, X\right)=c_{X}$ and that $\mathbb{R}_{\geq 0}\left[f_{i}\right]$ is an extremal ray of type $(n-1, n-2)^{s m}$, such that the target of its contraction is again Fano. This is Lemma 3.2.4.

In particular, this shows that $X$ has at least one extremal ray $R_{0}$ of type $(n-1, n-2)^{s m}$ such that if $E_{0}:=\operatorname{Locus}\left(R_{0}\right)$, then $\operatorname{codim} \mathcal{N}_{1}\left(E_{0}, X\right)=c_{X}$, and the target of the contraction of $R_{0}$ is Fano.

Now we replace $D$ by $E_{0}$, and apply again the same construction. Let $p: E_{0} \rightarrow F$ be the $\mathbb{P}^{1}$-bundle structure. Since $E_{1}, \ldots, E_{s}$ are pairwise disjoint, either $E_{0} \cap E_{i}$ is a union of fibers of $p$ for every $i=1, \ldots, s$, or $p\left(E_{0} \cap E_{i}\right)=F$ for every $i=1, \ldots, s$. The second preparatory step is to show that if $E_{1}, \ldots, E_{s}$ intersect $E_{0}$ horizontally with respect to the $\mathbb{P}^{1}$-bundle (i.e., $p\left(E_{0} \cap E_{i}\right)=F$ ), the divisors $E_{0}, \ldots, E_{s}$ have very special properties; in particular, for every $i=0, \ldots, s, E_{i} \cong \mathbb{P}^{1} \times F$ where $F$ is an $(n-2)$-dimensional Fano manifold. This is Lemma 3.2.7.

The third preparatory step is show that we can always choose the extremal ray $R_{0}$, and the special Mori program for $-E_{0}$, in such a way that $E_{1}, \ldots, E_{s}$ actually intersect $E_{0}$ horizontally with respect to the $\mathbb{P}^{1}$-bundle, so that the previous result applies. This is Lemma 3.2.10.

Then we are ready for the proof of Proposition 3.2.1. We use the properties given by Lemma 3.2.7 to show that $E_{1}, \ldots, E_{s}$ are the exceptional divisors of the blow-up 
$\sigma: X \rightarrow X_{s}$ of a Fano manifold $X_{s}$ in $s$ smooth codimension 2 subvarieties. Moreover there is an elementary contraction of fiber type $\varphi: X_{s} \rightarrow Y$ such that if $\psi:=\varphi \circ \sigma: X \rightarrow Y$, then $\psi\left(E_{0}\right)=Y$, and $\psi$ is finite on $\{p t\} \times F \subset E_{0}$ (recall that $E_{0} \cong \mathbb{P}^{1} \times F$ ). We have then two possibilities: either $\psi$ is not finite on $E_{0}$ and $\operatorname{dim} Y=n-2$, or $\psi$ is finite on $E_{0}$ and $\operatorname{dim} Y=n-1$.

We first consider the case where $\psi$ is not finite on $E_{0}$, in 3.2.21. We use the divisors $E_{0}, \ldots, E_{s}$ to define a contraction $X \rightarrow S$ onto a surface, such that the induced morphism $\pi: X \rightarrow S \times Y$ is finite. Finally we show that in fact $\pi$ is an isomorphism; here the key property is that $E_{0}, \ldots, E_{s}$ are products.

Then we consider in 3.2.24 the case where $\psi$ is finite on $E_{0}$. In this situation $Y$ is smooth, and both $\psi$ and $\varphi$ are conic bundles. If $T_{1}, \ldots, T_{s} \subset X_{s}$ are the subvarieties blown up by $\sigma$, the transforms $\widehat{E}_{1}, \ldots, \widehat{E}_{s} \subset X$ of $\varphi^{-1}\left(\varphi\left(T_{i}\right)\right)$ are smooth $\mathbb{P}^{1}$-bundles.

Similarly to what previously done for $E_{0}, \ldots, E_{s}$, we show that $\widehat{E}_{i} \cong \mathbb{P}^{1} \times F$ for every $i=1, \ldots, s$.

Since $\psi\left(E_{0}\right)=Y, Y$ is covered by the family of rational curves $\psi\left(\mathbb{P}^{1} \times\{p t\}\right)$. We use a result from [8] to show that in fact these rational curves are the fibers of a smooth morphism $Y \rightarrow Y^{\prime}$, where $\operatorname{dim} Y^{\prime}=n-2$.

In this way we get a contraction $X \rightarrow Y^{\prime}$, and we proceed similarly to the previous case: we use the divisors $E_{0}, E_{1}, \ldots, E_{s}, \widehat{E}_{1}, \ldots, \widehat{E}_{s}$ to define a contraction $X \rightarrow S$ onto a surface, and show that the induced morphism $X \rightarrow S \times Y^{\prime}$ is an isomorphism.

Let us start with the first preparatory result.

Lemma 3.2.4. - Let $X$ be a Fano manifold such that either $c_{X} \geq 4$, or $c_{X}=3$ and $X$ satisfies (3.6.a).

Let $D \subset X$ be a prime divisor with $\operatorname{codim} \mathcal{N}_{1}(D, X)=c_{X}$, consider a special Mori program for $-D$, and let $E_{1}, \ldots, E_{s} \subset X$ be the $\mathbb{P}^{1}$-bundles determined by the Mori program. For $i=1, \ldots$, s let $f_{i} \subset E_{i}$ be a fiber of the $\mathbb{P}^{1}$-bundle, and set $R_{i}:=\mathbb{R}_{\geq 0}\left[f_{i}\right]$. Then we have the following:

(1) $s \in\left\{c_{X}-1, c_{X}\right\}$ and $s \geq 3$;

(2) $R_{i}$ is an extremal ray of type $(n-1, n-2)^{\text {sm }}$, the target of the contraction of $R_{i}$ is Fano, and $\operatorname{codim} \mathcal{N}_{1}\left(E_{i}, X\right)=c_{X}$, for every $i=1, \ldots, s$;

(3) there exists a linear subspace $L \subset \mathcal{N}_{1}(X)$, of codimension $c_{X}+1$, such that

$$
L=\mathcal{N}_{1}\left(D \cap E_{i}, X\right)=\mathcal{N}_{1}(D, X) \cap E_{i}^{\perp}=\mathcal{N}_{1}\left(E_{i}, X\right) \cap E_{i}^{\perp} \text { for every } i=1, \ldots, s .
$$

We will call $R_{1}, \ldots, R_{s}$ the extremal rays determined by the special Mori program for $-D$ that we are considering. Notice that differently from the case of the $\mathbb{P}^{1}$-bundles $E_{1}, \ldots, E_{s}$, the extremal rays $R_{1}, \ldots, R_{s}$ are defined only when $X$ satisfies the assumptions of Lemma 3.2.4, and $D \subset X$ is a prime divisor with $\operatorname{codim} \mathcal{N}_{1}(D, X)=c_{X}$.

Proof. - We know by Lemma 2.7 that: $E_{i} \cdot f_{i}=-1$ and $D \cdot f_{i}>0$ for $i=1, \ldots, s$, $E_{1}, \ldots, E_{s}$ are pairwise disjoint, and $s \in\left\{c_{X}-1, c_{X}\right\}$ because $\operatorname{codim} \mathcal{N}_{1}(D, X)=c_{X}$. Moreover, if $c_{X}=3$, then $s=3$ by (3.6.a), so that in any case $s \geq 3$, and we get (1).

Therefore, by Lemma 3.1.8, we have codim $\mathcal{N}_{1}\left(E_{i}, X\right)=c_{X}$ and $\operatorname{codim} \mathcal{N}_{1}\left(D \cap E_{i}, X\right)=$ $c_{X}+1$ for every $i=1, \ldots, s$. In particular, Lemma 3.1.7 applies; let $L \subset \mathcal{N}_{1}(X)$ be the linear 
subspace such that codim $L=c_{X}+1$ and $L=\mathcal{N}_{1}\left(D \cap E_{i}, X\right)=\mathcal{N}_{1}(D, X) \cap E_{i}^{\perp}$ for every $i=1, \ldots, s$.

Fix $i \in\{1, \ldots, s\}$. Since $E_{i} \cdot f_{i}=-1$, we have $\mathcal{N}_{1}\left(E_{i}, X\right) \nsubseteq E_{i}^{\perp}$, therefore $\operatorname{dim} \mathcal{N}_{1}\left(E_{i}, X\right) \cap E_{i}^{\perp}=\operatorname{dim} \mathcal{N}_{1}\left(E_{i}, X\right)-1=\rho_{X}-c_{X}-1=\operatorname{dim} L$. On the other hand we have $L \subseteq E_{i}^{\perp}$ and $L=\mathcal{N}_{1}\left(D \cap E_{i}, X\right)$, in particular $L \subseteq \mathcal{N}_{1}\left(E_{i}, X\right)$. Thus $L \subseteq \mathcal{N}_{1}\left(E_{i}, X\right) \cap E_{i}^{\perp}$, so the two subspaces must coincide, and we get (3).

Finally, (2) follows from Remark 3.1.4 applied to $D$ and $E_{i}$.

Lemma 3.2.5. - Let $X$ be a Fano manifold such that either $c_{X} \geq 4$, or $c_{X}=3$ and $X$ satisfies (3.6.a).

Let $D \subset X$ be a prime divisor with $\operatorname{codim} \mathcal{N}_{1}(D, X)=c_{X}$, and $R$ an extremal ray of type $(n-1, n-2)^{\text {sm }}$ such that $D \cdot R>0, R \not \subset \mathcal{N}_{1}(D, X)$, and the target of the contraction of $R$ is Fano.

Set $E:=\operatorname{Locus}(R)$. Then $\mathcal{N}_{1}(D \cap E, X)=\mathcal{N}_{1}(D, X) \cap E^{\perp}=\mathcal{N}_{1}(E, X) \cap E^{\perp}$.

Proof. - Consider the contraction $\varphi: X \rightarrow Y$ of $R$, so that by the assumptions $Y$ is a Fano manifold, and consider the prime divisor $\varphi(D) \subset Y$.

By Proposition 2.4, there exists a special Mori program for $-\varphi(D)$ in $Y$. Together with $\varphi$, this gives a special Mori program for $-D$ in $X$, where the first extremal ray is precisely $Q_{0}=R$ :

$$
X \stackrel{\varphi}{\longrightarrow} Y=Y_{0} \stackrel{\sigma_{0}}{\rightarrow} Y_{1} \rightarrow \quad \cdots \quad \rightarrow Y_{k-1} \stackrel{\sigma_{k-1}}{-\rightarrow} Y_{k} .
$$

We apply Lemmas 2.7 and 3.2.4; since $R \not \subset \mathcal{N}_{1}(D, X), E$ is one of the $\mathbb{P}^{1}$-bundles determined by this special Mori program for $-D$. Thus the statement follows from 3.2.4(3).

Remark 3.2.6. - Let $X$ be a Fano manifold such that either $c_{X} \geq 4$, or $c_{X}=3$ and $X$ satisfies (3.6.a). Recall from Proposition 2.4 that there exists a special Mori program for any divisor in $X$.

The first consequence of Lemma 3.2.4 (applied to any prime divisor $D \subset X$ with $\left.\operatorname{codim} \mathcal{N}_{1}(D, X)=c_{X}\right)$ is that $X$ has an extremal ray $R_{0}$ of type $(n-1, n-2)^{s m}$ such that if $E_{0}:=\operatorname{Locus}\left(R_{0}\right)$, then codim $\mathcal{N}_{1}\left(E_{0}, X\right)=c_{X}$, and the target of the contraction of $R_{0}$ is Fano.

In particular, we can consider a special Mori program for $-E_{0}$, and apply again Lemma 3.2.4. Let $R_{1}, \ldots, R_{s}$ be the extremal rays determined by the Mori program, with loci $E_{1}, \ldots, E_{s}$. Since, by 2.7(3) and 2.7(4), $E_{1}, \ldots, E_{s}$ are pairwise disjoint and $E_{0} \neq E_{i}, E_{0} \cap E_{i} \neq \varnothing$ for $i=1, \ldots, s$, by Remark 3.1.6 we have two possibilities: either $E_{1} \cdot R_{0}=\cdots=E_{s} \cdot R_{0}=0$, or $E_{i} \cdot R_{0}>0$ for every $i=1, \ldots, s$.

In the next lemma we are going to show that in the second case (i.e., when $E_{1} \cdot R_{0}>0$ ) the extremal rays $R_{0}, \ldots, R_{s}$ have very special properties, in particular that the divisors $E_{0}, \ldots, E_{s}$ are products. 
Lemma 3.2.7. - Let $X$ be a Fano manifold such that either $c_{X} \geq 4$, or $c_{X}=3$ and $X$ satisfies (3.6.a).

Let $R_{0}$ be an extremal ray of $X$, of type $(n-1, n-2)^{\text {sm }}$, such that the target of the contraction of $R_{0}$ is Fano, and codim $\mathcal{N}_{1}\left(E_{0}, X\right)=c_{X}$, where $E_{0}:=\operatorname{Locus}\left(R_{0}\right)$.

Consider a special Mori program for $-E_{0}$, let $R_{1}, \ldots, R_{s}$ be the extremal rays determined by the Mori program, and set $E_{i}:=\operatorname{Locus}\left(R_{i}\right)$ for $i=1, \ldots, s$.

Assume that $E_{1} \cdot R_{0}>0$. Then we have the following:

(1) $\operatorname{codim} \mathcal{N}_{1}\left(E_{i}, X\right)=c_{X}$, and $E_{i} \cong \mathbb{P}^{1} \times F$ with $F$ an $(n-2)$-dimensional Fano manifold, for $i=0, \ldots, s$. We set $F_{i}:=\{p t\} \times F \subset E_{i}$;

(2) $R_{i}$ is the unique extremal ray of $X$ having negative intersection with $E_{i}$, and the target of the contraction of $R_{i}$ is Fano, for every $i=0, \ldots, s$;

(3) $E_{1}, \ldots, E_{s}$ are pairwise disjoint, and $E_{0} \cap E_{i}=\{p t s\} \times F$ for every $i=1, \ldots, s$;

(4) $E_{i} \cdot R_{0}>0$ and $E_{0} \cdot R_{i}>0$ for every $i=1, \ldots, s$;

(5) there exists a linear subspace $L \subset \mathcal{N}_{1}(X)$, of codimension $c_{X}+1$, such that

$$
L=\mathcal{N}_{1}\left(E_{0} \cap E_{i}, X\right)=\mathcal{N}_{1}\left(F_{j}, X\right) \text { and } \mathcal{N}_{1}\left(E_{j}, X\right)=\mathbb{R} R_{j} \oplus L
$$

for every $i=1, \ldots, s$ and $j=0, \ldots, s$, and moreover $\operatorname{dim}\left(\mathbb{R}\left(R_{0}+\cdots+R_{s}\right)+L\right)=$ $s+1+\operatorname{dim} L$

(6) $L \subseteq E_{0}^{\perp} \cap \cdots \cap E_{s}^{\perp}$, and equality holds if $s=c_{X}$.

Proof. - By 2.7(3) and 2.7(4) we know that $E_{0} \cdot R_{i}>0$ (in particular $E_{0} \neq E_{i}$ and $\left.E_{0} \cap E_{i} \neq \varnothing\right)$ and $R_{i} \not \subset \mathcal{N}_{1}\left(E_{0}, X\right)$ for $i=1, \ldots, s$, and that $E_{1}, \ldots, E_{s}$ are pairwise disjoint.

Secondly, Lemma 3.2.4 shows that $s \in\left\{c_{X}-1, c_{X}\right\}$ and $s \geq 3$, that $\operatorname{codim} \mathcal{N}_{1}\left(E_{i}, X\right)=c_{X}$ for $i=1, \ldots, s$, and that there exists a linear subspace $L \subset \mathcal{N}_{1}(X)$, of codimension $c_{X}+1$, such that

$$
L=\mathcal{N}_{1}\left(E_{0} \cap E_{i}, X\right)=\mathcal{N}_{1}\left(E_{0}, X\right) \cap E_{i}^{\perp}=\mathcal{N}_{1}\left(E_{i}, X\right) \cap E_{i}^{\perp}
$$

for every $i=1, \ldots, s$. Moreover Remark 3.1.6 yields $E_{i} \cdot R_{0}>0$ for every $i=1, \ldots, s$, because $E_{1} \cdot R_{0}>0$, so we get (4).

Fix $i \in\{1, \ldots, s\}$. We have $\operatorname{dim} \mathcal{N}_{1}\left(E_{0} \cap E_{i}, X\right)=\operatorname{dim} L=\rho_{X}-c_{X}-1<\rho_{X}-c_{X}=$ $\operatorname{dim} \mathcal{N}_{1}\left(E_{0}, X\right)$, and since $E_{i} \cdot R_{0}>0,3.1 .3(2)$ gives $R_{0} \not \subset \mathcal{N}_{1}\left(E_{0} \cap E_{i}, X\right)$. Moreover

$$
\mathcal{N}_{1}\left(E_{0} \cap E_{i}, X\right) \subseteq \mathcal{N}_{1}\left(E_{0}, X\right) \cap \mathcal{N}_{1}\left(E_{i}, X\right) \subsetneq \mathcal{N}_{1}\left(E_{0}, X\right)
$$

(because $R_{i} \not \subset \mathcal{N}_{1}\left(E_{0}, X\right)$ ), and since $\mathcal{N}_{1}\left(E_{0} \cap E_{i}, X\right)$ has codimension 1 in $\mathcal{N}_{1}\left(E_{0}, X\right)$, we deduce that $\mathcal{N}_{1}\left(E_{0} \cap E_{i}, X\right)=\mathcal{N}_{1}\left(E_{0}, X\right) \cap \mathcal{N}_{1}\left(E_{i}, X\right)$. This yields that $R_{0} \not \subset \mathcal{N}_{1}\left(E_{i}, X\right)$.

Now we can apply Lemma 3.2.5 to $E_{i}$ and $R_{0}$, and deduce that

$$
L=\mathcal{N}_{1}\left(E_{0} \cap E_{i}, X\right)=\mathcal{N}_{1}\left(E_{i}, X\right) \cap E_{0}^{\perp} .
$$

Thanks to (4), (3.2.8), and (3.2.9), we can use Lemma 3.1.5 to show (1). First of all we apply Lemma 3.1.5 with $D=E_{i}$ and $E=E_{0}$, and we deduce that $E_{0} \cong \mathbb{P}^{1} \times F$ where $F$ is an $(n-2)$-dimensional Fano manifold, and $E_{0} \cap E_{i}=\{p t s\} \times F \subset E_{0}$. Moreover we get (2) for $R_{0}$.

$4^{\text {e }}$ SÉRIE - TOME $45-2012-$ No $^{\circ}$ 
Then we apply Lemma 3.1.5 again, with $D=E_{0}$ and $E=E_{i}$, and we get $E_{i} \cong \mathbb{P}^{1} \times F^{i}$ and $E_{0} \cap E_{i}=\{p t s\} \times F^{i} \subset E_{i}$; in particular, $F^{i}=F$, and we have (3). Moreover we get (2) for $R_{i}$.

We have $L \subseteq E_{0}^{\perp} \cap \cdots \cap E_{s}^{\perp}$ by (3.2.8) and (3.2.9). To get (5), it is enough to show that $\left[f_{0}\right], \ldots,\left[f_{s}\right] \in \mathcal{N}_{1}(X)$ are linearly independent and that $\left(\mathbb{R}\left[f_{0}\right]+\cdots+\mathbb{R}\left[f_{s}\right]\right) \cap L=\{0\}$. So suppose that there exist $\lambda_{0}, \ldots, \lambda_{s} \in \mathbb{R}$ such that

$$
\sum_{i=0}^{s} \lambda_{i} f_{i} \in L .
$$

Intersecting with $E_{j}$ for $j \in\{1, \ldots, s\}$ we get $\lambda_{j}=\lambda_{0} E_{j} \cdot f_{0}$, and intersecting with $E_{0}$ we get $\lambda_{0}\left(\sum_{i=1}^{s}\left(E_{i} \cdot f_{0}\right)\left(E_{0} \cdot f_{i}\right)-1\right)=0$. Since $E_{i} \cdot f_{0}$ and $E_{0} \cdot f_{i}$ are positive integers by (4), and $s \geq 3$, we get $\lambda_{0}=0$ and hence $\lambda_{i}=0$ for $i=1, \ldots, s$, and we are done.

We are left to show (6). Similarly to what we have done for $\left[f_{0}\right], \ldots,\left[f_{s}\right]$, one checks that $\left[E_{0}\right], \ldots,\left[E_{s}\right]$ are linearly independent in $\mathcal{N}^{1}(X)$, so that $\operatorname{codim}\left(E_{0}^{\perp} \cap \cdots \cap E_{s}^{\perp}\right)=s+1$. Since $L \subseteq E_{0}^{\perp} \cap \cdots \cap E_{s}^{\perp}$ and $\operatorname{codim} L=c_{X}+1$, if $s=c_{X}$ the two subspaces coincide.

Lemma 3.2.10. - Let $X$ be a Fano manifold such that either $c_{X} \geq 4$, or $c_{X}=3$ and $X$ satisfies (3.6.a). Then $X$ has an extremal ray $R_{0}$ with the following properties:

$-R_{0}$ is of type $(n-1, n-2)^{s m}$, the target of the contraction of $R_{0}$ is Fano, and $\operatorname{codim} \mathcal{N}_{1}\left(E_{0}, X\right)=c_{X}$, where $E_{0}:=\operatorname{Locus}\left(R_{0}\right)$;

- there exists a special Mori program for $-E_{0}$ such that, if $R_{1}, \ldots, R_{s}$ are the extremal rays determined by the Mori program, we have $\operatorname{Locus}\left(R_{i}\right) \cdot R_{0}>0$ for every $i=1, \ldots, s$.

Proof. - Let $\phi=\left\{S^{1}, \ldots, S^{h}\right\}$ be an ordered set of extremal rays of $X$, and set $E^{i}:=\operatorname{Locus}\left(S^{i}\right)$. Consider the following properties:

(P1) $S^{i}$ is of type $(n-1, n-2)^{s m}$, the target of the contraction of $S^{i}$ is Fano, and $\operatorname{codim} \mathcal{N}_{1}\left(E^{i}, X\right)=c_{X}$, for every $i=1, \ldots, h$;

(P2) $E^{i-1} \cdot S^{i}>0$ and $S^{i} \not \subset \mathcal{N}_{1}\left(E^{i-1}, X\right)$, for every $i=2, \ldots, h$;

(P3) for every $1 \leq j<i \leq h$ we have $E^{i} \cdot S^{j}=0$ and $E^{i} \cap E^{j} \neq \varnothing$.

We notice first of all that by Remark 3.2.6, there exists an extremal ray $S^{1}$ of $X$, of type $(n-1, n-2)^{s m}$, such that codim Locus $\left(S^{1}\right)=c_{X}$, and the target of the contraction of $S^{1}$ is Fano. Then $\phi=\left\{S^{1}\right\}$ satisfies properties (P1), (P2), and (P3).

Consider now an arbitrary ordered set of extremal rays $\&=\left\{S^{1}, \ldots, S^{h}\right\}$ satisfying properties (P1), (P2), and (P3). We show that $h \leq \rho_{X}$.

Let $\gamma_{i} \in S^{i}$ be a non-zero element, for $i=1, \ldots, h$. We have $E^{i} \cdot \gamma_{i} \neq 0$ for every $i=1, \ldots, h$, and $E^{i} \cdot \gamma_{j}=0$ for every $1 \leq j<i \leq h$ by (P3). This shows that $\gamma_{1}, \ldots, \gamma_{h}$ are linearly independent in $\mathcal{N}_{1}(X)$ : indeed if there exist $a_{1}, \ldots, a_{h} \in \mathbb{R}$ such that $\sum_{i=1}^{h} a_{i} \gamma_{i}=0$, then intersecting with $E_{h}$ we get $a_{h}=0$, and so on. Thus $h \leq \rho_{X}$.

Then Lemma 3.2.10 is a consequence of the following claim.

Claim 3.2.11. - Assume that $\&=\left\{S^{1}, \ldots, S^{h}\right\}$ is an ordered set of extremal rays having properties (P1), (P2), and (P3). Then either $R_{0}:=S^{h}$ satisfies the statement of Lemma 3.2.10, or there exists an extremal ray $S^{h+1}$ such that $\phi^{\prime}:=\left\{S^{1}, \ldots, S^{h}, S^{h+1}\right\}$ still has properties (P1), (P2), and (P3). 
Proof of Claim 3.2.11. - By (P1) the ray $S^{h}$ is of type $(n-1, n-2)^{s m}$, the target of its contraction is Fano, and codim $\mathcal{N}_{1}\left(E^{h}, X\right)=c_{X}$. Consider a special Mori program for $-E^{h}$ (which exists by Proposition 2.4), and let $S_{1}^{h+1}, \ldots, S_{s}^{h+1}$ be the extremal rays determined by the Mori program, as in Lemma 3.2.4. Notice that $s \geq 3$ by 3.2.4(1). We set $E_{l}^{h+1}:=\operatorname{Locus}\left(S_{l}^{h+1}\right)$ for $l=1, \ldots, s$, so that $E_{1}^{h+1}, \ldots, E_{s}^{h+1}$ are the $\mathbb{P}^{1}$-bundles determined by the Mori program. By 2.7(3) we have

$$
E^{h} \cdot S_{l}^{h+1}>0 \text { and } S_{l}^{h+1} \not \subset \mathcal{N}_{1}\left(E^{h}, X\right) \text { for every } l=1, \ldots, s,
$$

and $E_{1}^{h+1}, \ldots, E_{s}^{h+1}$ are pairwise disjoint by 2.7(4).

Remark 3.2.6 shows that the intersections $E_{l}^{h+1} \cdot S^{h}$ (for $l=1, \ldots, s$ ) are either all zero, or all positive. In the latter case, $S^{h}$ satisfies the statement of Lemma 3.2.10.

Thus let us assume that $E_{1}^{h+1} \cdot S^{h}=\cdots=E_{s}^{h+1} \cdot S^{h}=0$, and set $S^{h+1}:=S_{1}^{h+1}$ and $E^{h+1}:=E_{1}^{h+1}$.

Since by assumption $\&$ has properties (P1) and (P2), in order to show that $\phi^{\prime}$ still satisfies (P1) and (P2), we just have to consider the case $i=h+1$. Then (P2) is given by (3.2.12), and (P1) follows from 3.2.4(2).

Now let us show the following:

$$
E_{l}^{h+1} \cdot S^{j}=0 \text { and } E_{l}^{h+1} \cap E^{j} \neq \varnothing \quad \text { for every } j=1, \ldots, h \text { and } l=1, \ldots, s .
$$

In particular, for $l=1,(3.2 .13)$ implies that $\phi^{\prime}$ satisfies (P3).

Let $l \in\{1, \ldots, s\}$. Since $E^{h} \cdot S_{l}^{h+1}>0$ by (3.2.12), we have $E^{h} \cap E_{l}^{h+1} \neq \varnothing$; moreover we have assumed that $E_{l}^{h+1} \cdot S^{h}=0$. Therefore (3.2.13) holds for $j=h$ and $l=1, \ldots, s$.

We proceed by decreasing induction on $j$ : we assume that (3.2.13) holds for some $j \in\{2, \ldots, h\}$ and for every $l=1, \ldots, s$, and we show that $E_{l}^{h+1} \cdot S^{j-1}=0$ and $E_{l}^{h+1} \cap E^{j-1} \neq \varnothing$ for every $l=1, \ldots, s$.

Fix $l \in\{1, \ldots, s\}$. Since $E_{l}^{h+1} \cdot S^{j}=0$ and $E_{l}^{h+1} \cap E^{j} \neq \varnothing$ by the induction assumption, $E_{l}^{h+1}$ contains a curve $C$ with class in $S^{j}$, in particular

$$
S^{j} \subset \mathcal{N}_{1}\left(E_{l}^{h+1}, X\right) \text {. }
$$

Since $E^{j-1} \cdot S^{j}>0$ by (P2), we have $E^{j-1} \cap C \neq \varnothing$ and hence $E_{l}^{h+1} \cap E^{j-1} \neq \varnothing$. Moreover $E_{l}^{h+1} \cdot S^{j}=0$ implies that $E_{l}^{h+1} \neq E^{j-1}$, thus $E_{l}^{h+1} \cdot S^{j-1} \geq 0$.

Recall from (P1) that $E^{j-1}$ is the locus of the extremal ray $S^{j-1}$, of type $(n-1, n-2)^{s m}$; in particular $E^{j-1}$ is a $\mathbb{P}^{1}$-bundle. Since $E_{1}^{h+1}, \ldots, E_{s}^{h+1}$ are pairwise disjoint, by Remark 3.1.6 the intersections $E_{l}^{h+1} \cdot S^{j-1}$ (for $l=1, \ldots, s$ ) are either all zero or all positive.

By contradiction, suppose that $E_{l}^{h+1} \cdot S^{j-1}>0$ for every $l=1, \ldots, s$. We have $\operatorname{codim} \mathcal{N}_{1}\left(E^{j-1}, X\right)=c_{X}$ by $(\mathrm{P} 1)$, hence 3.1.3(1) gives

$$
\operatorname{codim} \mathcal{N}_{1}\left(E^{j-1} \cap E_{l}^{h+1}, X\right) \leq \operatorname{codim} \mathcal{N}_{1}\left(E^{j-1}, X\right)+1=c_{X}+1 \text { for every } l=1, \ldots, s \text {. }
$$

Since $s \geq 3$, we can apply Lemma 3.1.7 to $E^{j-1}$ and $E_{1}^{h+1}, \ldots, E_{s}^{h+1}$, and deduce that $\operatorname{codim} \mathcal{N}_{1}\left(E^{j-1} \cap E^{h+1}, X\right)=c_{X}+1$ and $\mathcal{N}_{1}\left(E^{j-1} \cap E^{h+1}, X\right) \subseteq\left(E^{h+1}\right)^{\perp}$. In particular

$$
\mathcal{N}_{1}\left(E^{j-1} \cap E^{h+1}, X\right) \subseteq \mathcal{N}_{1}\left(E^{h+1}, X\right) \cap\left(E^{h+1}\right)^{\perp} .
$$

On the other hand $\mathcal{N}_{1}\left(E^{h+1}, X\right) \nsubseteq\left(E^{h+1}\right)^{\perp}$ because $E^{h+1} \cdot S^{h+1}<0$, therefore

$$
\operatorname{codim}\left(\mathcal{N}_{1}\left(E^{h+1}, X\right) \cap\left(E^{h+1}\right)^{\perp}\right)=c_{X}+1=\operatorname{codim} \mathcal{N}_{1}\left(E^{j-1} \cap E^{h+1}, X\right),
$$


and the two subspaces coincide.

By (3.2.14) and by the induction assumption we have $S^{j} \subset \mathcal{N}_{1}\left(E^{h+1}, X\right) \cap\left(E^{h+1}\right)^{\perp}$, therefore $S^{j} \subset \mathcal{N}_{1}\left(E^{j-1}, X\right)$, and this contradicts property (P2).

Proof of Proposition 3.2.1. - Let $R_{0}$ be the extremal ray of $X$ given by Lemma 3.2.10, and set $E_{0}:=\operatorname{Locus}\left(R_{0}\right)$. Then $\operatorname{codim} \mathcal{N}_{1}\left(E_{0}, X\right)=c_{X}$, and there exists a special Mori program for $-E_{0}$ which determines extremal rays $R_{1}, \ldots, R_{s}$ such that $E_{i} \cdot R_{0}>0$ for all $i=1, \ldots, s$, where $E_{i}:=\operatorname{Locus}\left(R_{i}\right)$. Thus Lemma 3.2.7 applies.

If $R$ is an extremal ray of $X$ different from $R_{1}, \ldots, R_{s}$, by 3.2.7(2) we have $E_{i} \cdot R \geq 0$ for every $i=1, \ldots, s$, hence $\left(-K_{X}+E_{1}+\cdots+E_{s}\right) \cdot R>0$. On the other hand $\left(-K_{X}+E_{1}+\cdots+E_{s}\right) \cdot R_{i}=0$ for every $i=1, \ldots, s$ (recall from 3.2.7(3) that $E_{1}, \ldots, E_{s}$ are pairwise disjoint), therefore $-K_{X}+E_{1}+\cdots+E_{s}$ is nef and

$$
\left(-K_{X}+E_{1}+\cdots+E_{s}\right)^{\perp} \cap \mathrm{NE}(X)=R_{1}+\cdots+R_{s}
$$

is a face of $\mathrm{NE}(X)$, of dimension $s$ by 3.2.7(5).

Let $\sigma: X \rightarrow X_{s}$ be the associated contraction, so that $\operatorname{ker} \sigma_{*}=\mathbb{R}\left(R_{1}+\cdots+R_{s}\right)$. Since $E_{1}, \ldots, E_{s}$ are pairwise disjoint, we see that $\operatorname{Exc}(\sigma)=E_{1} \cup \cdots \cup E_{s}, X_{s}$ is smooth, and $\sigma$ is the blow-up of $s$ smooth, pairwise disjoint, irreducible subvarieties $T_{1}, \ldots, T_{s} \subset X_{s}$ of codimension 2, where $T_{i}:=\sigma\left(E_{i}\right)$ for $i=1, \ldots, s$. Moreover $X_{s}$ is again Fano, because $-K_{X}+E_{1}+\cdots+E_{s}=\sigma^{*}\left(-K_{X_{s}}\right)$. Recall from 3.2.7(1) that $E_{i} \cong \mathbb{P}^{1} \times F$, and notice that $\sigma_{\mid E_{i}}$ is the projection onto $F \cong T_{i}$.

Set $\left(E_{0}\right)_{s}:=\sigma\left(E_{0}\right) \subset X_{s}$. Since $E_{0} \cong \mathbb{P}^{1} \times F$ and $E_{0} \cap E_{i}=\{p t s\} \times F$ for $i=1, \ldots, s$ by 3.2.7(1) and 3.2.7(3), the morphism $\sigma_{\mid E_{0}}: E_{0} \rightarrow\left(E_{0}\right)_{s}$ is birational and finite, i.e., it is the normalization. Moreover for $i=1, \ldots, s$ we have $T_{i}=\sigma\left(E_{0} \cap E_{i}\right) \subset\left(E_{0}\right)_{s}$, so that

$$
\mathcal{N}_{1}\left(T_{i}, X_{s}\right)=\sigma_{*}\left(\mathcal{N}_{1}\left(E_{0} \cap E_{i}, X\right)\right)=\sigma_{*}(L),
$$

where $L \subset \mathcal{N}_{1}(X)$ is the linear subspace defined in 3.2.7(5). Again by 3.2.7(5) we know that $\mathcal{N}_{1}\left(E_{0}, X\right)=\mathbb{R} R_{0} \oplus L$, and that $\operatorname{dim}\left(\operatorname{ker} \sigma_{*}+\mathcal{N}_{1}\left(E_{0}, X\right)\right)=\operatorname{dim} \operatorname{ker} \sigma_{*}+\operatorname{dim} \mathcal{N}_{1}\left(E_{0}, X\right)$, therefore:

$$
\operatorname{ker} \sigma_{*} \cap \mathcal{N}_{1}\left(E_{0}, X\right)=\{0\} \text { and } \mathcal{N}_{1}\left(\left(E_{0}\right)_{s}, X_{s}\right)=\mathbb{R} \sigma_{*}\left(R_{0}\right) \oplus \sigma_{*}(L) \text {. }
$$

Finally, since $\sigma^{*}\left(\left(E_{0}\right)_{s}\right)=E_{0}+\sum_{i=1}^{s}\left(E_{0} \cdot f_{i}\right) E_{i}$ (as usual we denote by $f_{i} \subseteq E_{i}$ a fiber of the $\mathbb{P}^{1}$-bundle), by 3.2.7(4) and 3.2.7(6) we see that

$$
\left(E_{0}\right)_{s} \cdot \sigma\left(f_{0}\right)=\sum_{i=1}^{s}\left(E_{0} \cdot f_{i}\right)\left(E_{i} \cdot f_{0}\right)-1>0 \text { and } \sigma_{*}(L) \subseteq\left(E_{0}\right)_{s}^{\perp}
$$

(recall that $s \geq 3$ and $s \in\left\{c_{X}-1, c_{X}\right\}$ by 3.2.4(1)).

Factoring $\sigma$ as a sequence of $s$ blow-ups, we can view $\sigma: X \rightarrow X_{s}$ as a part of a special Mori program for $-E_{0}$ in $X$, with $s$ steps, and by (3.2.16) at each step we have $Q_{i} \not \subset \mathcal{N}_{1}\left(\left(E_{0}\right)_{i}, X_{i}\right)$. In particular 2.6(3) yields that

$$
\operatorname{codim} \mathcal{N}_{1}\left(\left(E_{0}\right)_{s}, X_{s}\right)=\operatorname{codim} \mathcal{N}_{1}\left(E_{0}, X\right)-s=c_{X}-s,
$$

hence either $s=c_{X}$ and $\mathcal{N}_{1}\left(\left(E_{0}\right)_{s}, X_{s}\right)=\mathcal{N}_{1}\left(X_{s}\right)$, or $s=c_{X}-1$ and $\operatorname{codim} \mathcal{N}_{1}\left(\left(E_{0}\right)_{s}, X_{s}\right)=1$. 
3.2.18. - Suppose that there exists an extremal ray $R$ of $X_{s}$ with $\left(E_{0}\right)_{s} \cdot R>0$ and $\operatorname{Locus}(R) \subsetneq X_{s}$. Then $s=c_{X}-1$ and $R \not \subset \mathcal{N}_{1}\left(\left(E_{0}\right)_{s}, X_{s}\right)$.

Since we have shown that $\mathcal{N}_{1}\left(\left(E_{0}\right)_{s}, X_{s}\right)=\mathcal{N}_{1}\left(X_{s}\right)$ when $s=c_{X}$, it is enough to show that $R \not \subset \mathcal{N}_{1}\left(\left(E_{0}\right)_{s}, X_{s}\right)$.

We first show that $R \not \subset \mathrm{NE}\left(\left(E_{0}\right)_{s}, X_{s}\right)$. Otherwise, since $\mathrm{NE}\left(\left(E_{0}\right)_{s}, X_{s}\right) \subseteq \mathrm{NE}\left(X_{s}\right)$, $R$ should be a one-dimensional face of $\mathrm{NE}\left(\left(E_{0}\right)_{s}, X_{s}\right)$. We have $\mathrm{NE}\left(E_{0}, X\right)=R_{0}+\mathrm{NE}\left(F_{0}, X\right)$ and $\mathrm{NE}\left(\left(E_{0}\right)_{s}, X_{s}\right)=\sigma_{*}\left(R_{0}\right)+\sigma_{*}\left(\mathrm{NE}\left(F_{0}, X\right)\right)$. On the other hand 3.2.7(5) and (3.2.17) give

$$
\sigma_{*}\left(\mathrm{NE}\left(F_{0}, X\right)\right) \subset \sigma_{*}\left(\mathcal{N}_{1}\left(F_{0}, X\right)\right)=\sigma_{*}(L) \subseteq\left(E_{0}\right)_{s}^{\perp},
$$

while $\left(E_{0}\right)_{s} \cdot R>0$, therefore we get $R=\sigma_{*}\left(R_{0}\right)$. But $\left(E_{0}\right)_{s}$ is covered by the curves $\sigma\left(f_{0}\right)$, so that $\operatorname{Locus}(R) \supseteq\left(E_{0}\right)_{s}$, which is impossible.

Therefore $R \not \subset \mathrm{NE}\left(\left(E_{0}\right)_{s}, X_{s}\right)$, and in particular the contraction of $R$ is finite on $\left(E_{0}\right)_{s}$. Since $\left(E_{0}\right)_{s} \cdot R>0$, this means that the contraction of $R$ has fibers of dimension $\leq 1$, therefore $R$ is of type $(n-1, n-2)^{s m}$ by [1, Theorem 2.3] and [21, Theorem 1.2].

In particular, $E_{R}:=\operatorname{Locus}(R)$ is a prime divisor covered by curves of anticanonical degree 1. Moreover these curves have class in $R$, thus they cannot be contained in $T_{1} \cup \cdots \cup T_{s}$, because $T_{1} \cup \cdots \cup T_{s} \subset\left(E_{0}\right)_{s}$. By a standard argument (see for instance [10, Remark 2.3]) we deduce that $E_{R} \cap\left(T_{1} \cup \cdots \cup T_{s}\right)=\varnothing$, hence by (3.2.15) and Remark 3.1.2 we have

$$
\sigma_{*}(L)=\mathcal{N}_{1}\left(T_{1}, X_{s}\right) \subseteq E_{R}^{\perp}
$$

Moreover $E_{R} \cdot \sigma\left(f_{0}\right) \geq 0$, because $E_{R} \neq\left(E_{0}\right)_{s}$ (as $\left.\left(E_{0}\right)_{s} \cdot R>0\right)$.

We show that $R \not \subset \mathcal{N}_{1}\left(\left(E_{0}\right)_{s}, X_{s}\right)$. By contradiction, suppose that $R \subset \mathcal{N}_{1}\left(\left(E_{0}\right)_{s}, X_{s}\right)$, and let $C$ be an irreducible curve with class in $R$. Then by (3.2.16) we have $[C]=\lambda\left[\sigma\left(f_{0}\right)\right]+\gamma$, with $\lambda \in \mathbb{R}$ and $\gamma \in \sigma_{*}(L)$. Using (3.2.17) we get $0<\left(E_{0}\right)_{s} \cdot C=\lambda\left(E_{0}\right)_{s} \cdot \sigma\left(f_{0}\right)$ and $\left(E_{0}\right)_{s} \cdot \sigma\left(f_{0}\right)>0$, thus $\lambda>0$. On the other hand $-1=E_{R} \cdot C=\lambda E_{R} \cdot \sigma\left(f_{0}\right)$, which gives a contradiction. Thus $R \not \subset \mathcal{N}_{1}\left(\left(E_{0}\right)_{s}, X_{s}\right)$.

3.2.19. - We show that we can assume that there exists an extremal ray $R$ of $X_{s}$ such that $\left(E_{0}\right)_{s} \cdot R>0$ and $\operatorname{Locus}(R)=X_{s}$.

This is clear if $s=c_{X}$, by 3.2.18. Suppose that $s=c_{X}-1$, and consider an extremal ray $R$ of $X_{s}$ with $\left(E_{0}\right)_{c_{X}-1} \cdot R>0$. If $\operatorname{Locus}(R)=X_{c_{X}-1}$, we are done; otherwise, by 3.2.18, we have $R \not \subset \mathcal{N}_{1}\left(\left(E_{0}\right)_{c_{X}-1}, X_{c_{X}-1}\right)$.

Let $\sigma_{c_{X}-1}: X_{c_{X}-1} \rightarrow X_{c_{X}}$ be the contraction of $R$, and consider the sequence

$$
X \stackrel{\sigma}{\longrightarrow} X_{c_{X}-1} \stackrel{\sigma_{c_{X}-1}^{\longrightarrow}}{\longrightarrow} X_{c_{X}} .
$$

Again, factoring $\sigma$ as a sequence of $c_{X}-1$ blow-ups, we can view this as a part of a special Mori program for $-E_{0}$ in $X$, with $c_{X}$ steps, and at each step $Q_{i} \not \subset \mathcal{N}_{1}\left(\left(E_{0}\right)_{i}, X_{i}\right)$.

The $\mathbb{P}^{1}$-bundles determined by this special Mori program are $E_{1}, \ldots, E_{c_{X}-1}$, and the transform of $E_{R}$ in $X$; the associated extremal rays (see Lemma 3.2.4) are $R_{1}, \ldots, R_{c_{X}-1}$, and an additional extremal ray $R_{c_{X}}$.

Since $E_{1} \cdot R_{0}>0$, Lemma 3.2.7 still applies, thus we can just replace $R_{1}, \ldots, R_{c_{X}-1}$ with $R_{1}, \ldots, R_{c_{X}}$, and restart. Since now the extremal rays are $c_{X}$ (instead of $c_{X}-1$ ), we are done by what precedes. 
3.2.20. - By 3.2.19 there exists an elementary contraction of fiber type $\varphi: X_{s} \rightarrow Y$ such that $\left(E_{0}\right)_{s} \cdot \mathrm{NE}(\varphi)>0$; set $\psi:=\varphi \circ \sigma: X \rightarrow Y$, and notice that $\varphi\left(\left(E_{0}\right)_{s}\right)=\psi\left(E_{0}\right)=Y$.

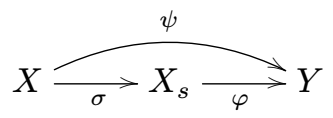

The sequence above is a Mori program for $-E_{0}$, with $s$ steps, and at each step $Q_{i} \not \subset \mathcal{N}_{1}\left(\left(E_{0}\right)_{i}, X_{i}\right)$. By 2.7(2) we have two possibilities: either $\mathcal{N}_{1}\left(\left(E_{0}\right)_{s}, X_{s}\right)=\mathcal{N}_{1}\left(X_{s}\right)$ and $s=c_{X}$, or $\mathrm{NE}(\varphi) \not \subset \mathcal{N}_{1}\left(\left(E_{0}\right)_{s}, X_{s}\right)$ and $s=c_{X}-1$.

Since $\mathcal{N}_{1}\left(T_{1}, X_{s}\right) \subseteq\left(E_{0}\right)_{s}^{\perp}$ by (3.2.15) and (3.2.17), $\varphi$ must be finite on $T_{1}$, so that $\operatorname{dim} Y \geq n-2$.

3.2.21. - First case: $\varphi$ is not finite on $\left(E_{0}\right)_{s}$. In this case $\operatorname{NE}(\varphi) \subset \mathcal{N}_{1}\left(\left(E_{0}\right)_{s}, X_{s}\right)$, therefore $\mathcal{N}_{1}\left(\left(E_{0}\right)_{s}, X_{s}\right)=\mathcal{N}_{1}\left(X_{s}\right)$ and $s=c_{X}$. This also shows that $L=E_{0}^{\perp} \cap \cdots \cap E_{c_{X}}^{\perp}$, by 3.2.7(6).

Recall that $Y=\psi\left(E_{0}\right)$, and that $E_{0} \cong \mathbb{P}^{1} \times F$ is smooth and Fano by 3.2.7(1). Moreover if $F_{0}:=\{p t\} \times F \subset E_{0}$, then $\mathcal{N}_{1}\left(F_{0}, X\right)=L$ by 3.2.7(5). Finally $\mathcal{N}_{1}\left(\sigma\left(F_{0}\right), X_{c_{X}}\right)=$ $\sigma_{*}(L) \subseteq\left(E_{0}\right)_{c_{X}}^{\perp}$ by (3.2.17), so that $\varphi$ is finite on $\sigma\left(F_{0}\right)$. Since $\sigma$ is finite on $E_{0}$, we deduce that $\psi$ is finite on $F_{0}$.

Let $E_{0} \stackrel{\alpha}{\rightarrow} \widetilde{Y} \rightarrow Y$ be the Stein factorization of $\psi_{\mid E_{0}}$. Since $\varphi$ is not finite on $\left(E_{0}\right)_{c_{X}}, \alpha$ is a non-trivial contraction of $E_{0}$. On the other hand $\alpha$ is finite on $F_{0}$ : the only possibility is that $\widetilde{Y} \cong F$ and $\alpha$ is the projection.

We deduce that $\operatorname{dim} Y=n-2$ and that $\psi$ contracts $f_{0}$, hence $\operatorname{NE}(\varphi)=\sigma_{*}\left(R_{0}\right)$. Thus $\mathrm{NE}(\psi)$ is a $\left(c_{X}+1\right)$-dimensional face of $\mathrm{NE}(X)$ containing $R_{0}, \ldots, R_{c_{X}}$; in particular $\rho_{Y}=\rho_{X}-c_{X}-1$.

Let us consider the divisor

$$
H:=2 E_{0}+\sum_{i=1}^{c_{X}} E_{i}
$$

on $X$. By 3.2.7(4) we have $H \cdot R_{i}>0$ for every $i=0, \ldots, c_{X}$, and

$$
L=E_{0}^{\perp} \cap \cdots \cap E_{c_{X}}^{\perp} \subseteq H^{\perp} .
$$

Recall from 3.2.7(1) and 3.2.7(5) that for every $i=0, \ldots, c_{X}$ we have $E_{i} \cong \mathbb{P}^{1} \times F$, and if $F_{i}:=\{p t\} \times F \subset E_{i}$, then $\mathcal{N}_{1}\left(F_{i}, X\right)=L \subset H^{\perp}$. In particular $\mathrm{NE}\left(E_{i}, X\right)=$ $R_{i}+\mathrm{NE}\left(F_{i}, X\right) \subset R_{i}+L$.

Let $C \subset X$ be an irreducible curve with $C \subset \operatorname{Supp} H=E_{0} \cup \cdots \cup E_{c_{X}}$. Then $C \subseteq E_{i}$ for some $i \in\left\{0, \ldots, c_{X}\right\}$, hence $[C] \in R_{i}+L$ and $H \cdot C \geq 0$.

On the other hand, since $H$ is effective, we have $H \cdot C^{\prime} \geq 0$ for every irreducible curve $C^{\prime}$ not contained in Supp $H$. Therefore $H$ is nef and defines a contraction $\xi: X \rightarrow S$ such that $\mathrm{NE}(\xi)=H^{\perp} \cap \mathrm{NE}(X)$.

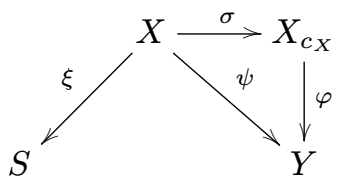

Let $i \in\left\{0, \ldots, c_{X}\right\}$. Since $\mathcal{N}_{1}\left(F_{i}, X\right) \subset H^{\perp}$, the image $\xi\left(F_{i}\right)$ is a point, and $\xi\left(E_{i}\right)=\xi\left(f_{i}\right)$ is an irreducible rational curve (because $H \cdot f_{i}>0$ ). Therefore $\xi_{\mid E_{i}}: E_{i} \rightarrow \xi\left(f_{i}\right)$ factors 
through the projection $E_{i} \rightarrow \mathbb{P}^{1}$. In particular $\operatorname{dim} \xi(\operatorname{Supp} H)=1$, hence $S$ is a surface by 3.1.1(1).

Let us show that

$$
\mathrm{NE}(\xi)=L \cap \mathrm{NE}(X)
$$

We already have $\mathrm{NE}(\xi)=H^{\perp} \cap \mathrm{NE}(X) \supseteq L \cap \mathrm{NE}(X)$. Conversely, let $C_{1} \subset X$ be an irreducible curve such that $\xi\left(C_{1}\right)=\{p t\}$, i.e., $H \cdot C_{1}=0$.

If $C_{1}$ is disjoint from Supp $H=E_{0} \cup \cdots \cup E_{c_{X}}$, then $C_{1} \cdot E_{i}=0$ for $i=0, \ldots, c_{X}$, hence $\left[C_{1}\right] \in L$.

If instead $C_{1}$ intersects $E_{0} \cup \cdots \cup E_{c_{X}}$, then it must be contained in it, and we have $C_{1} \subset E_{i}$ for some $i$. Since $\xi_{\mid E_{i}}$ factors as the projection onto $\mathbb{P}^{1}$ followed by a finite map, we get $C_{1} \subset F_{i}$, and again $\left[C_{1}\right] \in \mathcal{N}_{1}\left(F_{i}, X\right)=L$. Therefore we have (3.2.22).

In particular, for every $i=0, \ldots, c_{X}$ we have $\mathrm{NE}(\xi) \subseteq E_{i}^{\perp}$, therefore $E_{i}=\xi^{*}\left(\xi\left(E_{i}\right)\right)$ by 3.1.1(2).

Let $\pi: X \rightarrow S \times Y$ be the morphism induced by $\xi$ and $\psi$. We have $\operatorname{ker} \psi_{*}=\mathbb{R}\left(R_{0}+\cdots+R_{c_{X}}\right)$, and $\operatorname{ker} \psi_{*} \cap L=\{0\}$ by 3.2.7(5). Moreover ker $\xi_{*} \subseteq L$ by (3.2.22), therefore $\pi$ is finite.

In particular, $\xi$ must be equidimensional, hence $S$ is smooth by [2, Proposition 1.4.1] and [10, Lemma 3.10]. We need the following remark.

REMARK 3.2.23. - Let $W$ be a smooth Fano variety and suppose we have two contractions

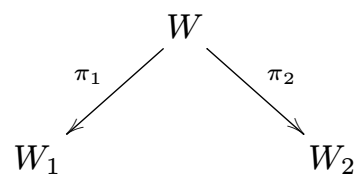

such that $W_{1}$ is smooth and the induced morphism $\pi: W \rightarrow W_{1} \times W_{2}$ is finite. Consider the relative canonical divisor $K_{W / W_{1}}:=K_{W}-\pi_{1}^{*} K_{W_{1}}$. If $\operatorname{ker}\left(\pi_{2}\right)_{*} \subseteq\left(K_{W / W_{1}}\right)^{\perp}$ in $\mathcal{N}_{1}(W)$, then $\pi$ is an isomorphism.

This is rather standard, we give a proof for the reader's convenience. Let $d$ be the degree of $\pi$, and $F \subset W$ a general fiber of $\pi_{2}$; the restriction $f:=\left(\pi_{1}\right)_{\mid F}: F \rightarrow W_{1}$ is finite of degree $d$. We observe that $F$ is Fano, hence numerical and linear equivalence for divisors in $F$ coincide, and by assumption $\left(K_{W / W_{1}}\right)_{\mid F} \equiv 0$. Then

$$
K_{F}=\left(K_{W}\right)_{\mid F}=\left(\pi_{1}^{*} K_{W_{1}}\right)_{\mid F}=f^{*} K_{W_{1}},
$$

so that $f$ is étale. Therefore $W_{1}$ is Fano too, in particular it is simply connected, thus $f$ is an isomorphism and $d=1$.

We carry on with the proof of Proposition 3.2.1. We want to apply Remark 3.2.23 to deduce that $\pi: X \rightarrow S \times Y$ is an isomorphism; for this we just need to show that $K_{X / S} \cdot R_{i}=0$ for $i=0, \ldots, c_{X}$, because $\operatorname{ker} \psi_{*}=\mathbb{R}\left(R_{0}+\cdots+R_{c_{X}}\right)$. But this follows easily because $E_{i}$ are products.

Indeed since both $S$ and $E_{i}$ are smooth, 3.1.1(4) yields that $\xi\left(E_{i}\right)$ is a smooth curve. Therefore $\xi\left(E_{i}\right) \cong \mathbb{P}^{1}$ and $\xi_{\mid E_{i}}$ is the projection, hence

$$
K_{X / S} \cdot f_{i}=\left(K_{X / S}\right)_{\mid E_{i}} \cdot f_{i}=K_{E_{i} / \xi\left(E_{i}\right)} \cdot f_{i}=0 .
$$

$4^{\text {e }}$ SÉRIE - TOME $45-2012$ - No 3 
Thus we conclude that $\pi$ is an isomorphism and $X \cong S \times Y$. Moreover since $\rho_{Y}=\rho_{X}-c_{X}-1$, we have $\rho_{S}=c_{X}+1$.

3.2.24. - Second case: $\varphi$ is finite on $\left(E_{0}\right)_{s}$. Then $\operatorname{dim} Y=n-1$ and every fiber of $\varphi$ is one-dimensional; moreover every fiber of $\psi$ has an irreducible component of dimension 1. Since $X$ and $X_{s}$ are Fano, [3, Lemma 2.12 and Theorem 4.1] show that $Y$ is smooth and that $\varphi$ and $\psi$ are conic bundles.

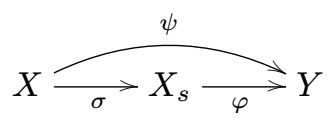

Set $Z_{i}:=\varphi\left(T_{i}\right)=\psi\left(E_{i}\right) \subset Y$ for $i=1, \ldots, s$. By standard arguments on conic bundles (as at the end of the proof of Lemma 2.8), we see that $Z_{1}, \ldots, Z_{s} \subset Y$ are pairwise disjoint smooth prime divisors, and that $\varphi$ is smooth over $Z_{1} \cup \cdots \cup Z_{s}$. For $i=1, \ldots, s$ let $\widehat{E}_{i} \subset X$ be the transform of $\varphi^{-1}\left(Z_{i}\right) \subset X_{s}$, so that $\psi^{-1}\left(Z_{i}\right)=E_{i} \cup \widehat{E}_{i}$. Then $\widehat{E}_{i}$ is a smooth $\mathbb{P}^{1}$-bundle with fiber $\widehat{f}_{i} \subset \widehat{E}_{i}$, such that $\widehat{E}_{i} \cdot \widehat{f}_{i}=-1$. Moreover $f_{i}+\widehat{f}_{i}$ is numerically equivalent to a general fiber of $\psi$, and $E_{i} \cdot \widehat{f}_{i}=\widehat{E}_{i} \cdot f_{i}=1$.

In particular, the divisors $E_{0}, E_{1}, \ldots, E_{s}, \widehat{E}_{1}, \ldots, \widehat{E}_{s}$ are all distinct (recall that $\psi\left(E_{0}\right)=Y$ ), and $E_{1} \cup \widehat{E}_{1}, \ldots, E_{s} \cup \widehat{E}_{s}$ are pairwise disjoint.

Let us show that $\left[E_{0}\right],\left[E_{1}\right], \ldots,\left[E_{s}\right],\left[\widehat{E}_{1}\right]$ are linearly independent in $\mathcal{N}^{1}(X)$. Indeed suppose that

$$
a E_{0}+\sum_{i=1}^{s} b_{i} E_{i}+d \widehat{E}_{1} \equiv 0,
$$

with $a, b_{i}, d \in \mathbb{R}$. Intersecting with a general fiber of $\psi: X \rightarrow Y$, we get $a=0$. Intersecting with $f_{2}, \ldots, f_{s}$, we get $b_{2}=\cdots=b_{s}=0$. Finally intersecting with $f_{1}$ we get $d=b_{1}$, that is, $d\left(E_{1}+\widehat{E}_{1}\right) \equiv 0$, which yields $d=0$, and we are done.

If $i, j \in\{1, \ldots, s\}$ with $i \neq j$, we have $E_{i} \cap \widehat{E}_{j}=\varnothing$, and hence $L \subseteq \mathcal{N}_{1}\left(E_{i}, X\right) \subseteq \widehat{E}_{j}^{\perp}$ (see Remark 3.1.2). Therefore by 3.2.7(6)

$$
L \subseteq E_{0}^{\perp} \cap E_{1}^{\perp} \cap \cdots \cap E_{s}^{\perp} \cap \widehat{E}_{1}^{\perp} \cap \cdots \cap \widehat{E}_{s}^{\perp} \subseteq E_{0}^{\perp} \cap E_{1}^{\perp} \cap \cdots \cap E_{s}^{\perp} \cap \widehat{E}_{1}^{\perp} .
$$

Since the classes of $E_{0}, \ldots, E_{s}, \widehat{E}_{1}$ in $\mathcal{N}^{1}(X)$ are linearly independent and $s \geq c_{X}-1$, we get

$$
c_{X}+1=\operatorname{codim} L \geq s+2 \geq c_{X}+1,
$$

which yields $s=c_{X}-1$ and

$$
L=E_{0}^{\perp} \cap E_{1}^{\perp} \cap \cdots \cap E_{c_{X}-1}^{\perp} \cap \widehat{E}_{1}^{\perp}=E_{0}^{\perp} \cap E_{1}^{\perp} \cap \cdots \cap E_{c_{X}-1}^{\perp} \cap \widehat{E}_{1}^{\perp} \cap \cdots \cap \widehat{E}_{c_{X}-1}^{\perp} .
$$

Let $i \in\left\{1, \ldots, c_{X}-1\right\}$. Observe that $\left[\widehat{f}_{i}\right] \notin \mathcal{N}_{1}\left(E_{i}, X\right)$ : otherwise by 3.2.7(5) we would have $\widehat{f}_{i} \equiv \lambda f_{i}+\gamma$, with $\lambda \in \mathbb{R}$ and $\gamma \in L \subset E_{0}^{\perp} \cap E_{i}^{\perp}$. Intersecting with $E_{i}$ we get $\lambda=-1$, hence $E_{0} \cdot \widehat{f}_{i}=-E_{0} \cdot f_{i}<0$, which is impossible because $E_{0} \neq \widehat{E}_{i}$. We also notice that $E_{0}$ cannot contain any curve $\widehat{f}_{i}$, because $\sigma\left(\widehat{f}_{i}\right)$ is a fiber of $\varphi$, and $\varphi$ is finite on $\left(E_{0}\right)_{c_{X}-1}=\sigma\left(E_{0}\right)$.

Therefore we can apply Lemma 3.1.10 to $E_{0}$ and $E_{1}, \ldots, E_{c_{X}-1}, \widehat{E}_{1}, \ldots, \widehat{E}_{c_{X}-1}$, and we get:

$\operatorname{codim} \mathcal{N}_{1}\left(\widehat{E}_{i}, X\right)=c_{X}$ and $R_{i} \not \subset \mathcal{N}_{1}\left(\widehat{E}_{i}, X\right)$ for every $i=1, \ldots, c_{X}-1$ 
Fix again $i \in\left\{1, \ldots, c_{X}-1\right\}$. Lemma 3.2.5, applied to $\widehat{E}_{i}$ and $R_{i}$, yields that

$$
\mathcal{N}_{1}\left(E_{i} \cap \widehat{E}_{i}, X\right)=\mathcal{N}_{1}\left(\widehat{E}_{i}, X\right) \cap E_{i}^{\perp}=\mathcal{N}_{1}\left(E_{i}, X\right) \cap E_{i}^{\perp}=L
$$

(see (3.2.8) for the last equality). Finally we apply Lemma 3.1 .5 to $D=E_{i}$ and $E=\widehat{E}_{i}$, and we deduce that $\widehat{R}_{i}:=\mathbb{R}_{\geq 0}\left[\widehat{f}_{i}\right]$ is an extremal ray of type $(n-1, n-2)^{s m}, \widehat{E}_{i} \cong \mathbb{P}^{1} \times \widehat{F}^{i}$, and $E_{i} \cap \widehat{E}_{i}=\{p t s\} \times \widehat{F}^{i} \subset \widehat{E}_{i}$. On the other hand again Lemma 3.1.5, applied now to $D=\widehat{E}_{i}$ and $E=E_{i}$, shows that $E_{i} \cap \widehat{E}_{i}=\{p t s\} \times F \subset E_{i} \cong \mathbb{P}^{1} \times F$, hence $\widehat{F}^{i}=F$.

Observe that $\mathrm{NE}(\psi)=R_{1}+\widehat{R}_{1}+\cdots+R_{c_{X}-1}+\widehat{R}_{c_{X}-1}$ has dimension $c_{X}$, and that $\psi_{\mid E_{0}}: E_{0} \cong \mathbb{P}^{1} \times F_{0} \rightarrow Y$ is finite. We need the following lemma.

Lemma 3.2.25. - Let $E$ be a projective manifold and $\pi: E \rightarrow W$ a $\mathbb{P}^{1}$-bundle with fiber $f \subset E$. Moreover let $\psi_{0}: E \rightarrow Y$ be a morphism onto a projective manifold $Y$, such that $\operatorname{dim} \psi_{0}(f)=1$. Suppose that there exists a prime divisor $Z_{1} \subset Y$ such that $\mathcal{N}_{1}\left(Z_{1}, Y\right) \subsetneq \mathcal{N}_{1}(Y)$ and $\psi_{0}^{*}\left(Z_{1}\right) \cdot f>0$. Then there is a commutative diagram:

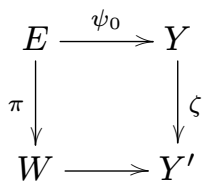

where $Y^{\prime}$ is smooth and $\zeta$ is a smooth morphism with fibers isomorphic to $\mathbb{P}^{1}$.

Proof of Lemma 3.2.25. - Consider the morphism $\phi: E \rightarrow W \times Y$ induced by $\pi$ and $\psi_{0}$, set $E^{\prime}:=\phi(E) \subset W \times Y$, and let $\pi^{\prime}: E^{\prime} \rightarrow W$ be the projection. For every $p \in W$ we have $\pi^{-1}(p)=\phi^{-1}\left(\left(\pi^{\prime}\right)^{-1}(p)\right)$, hence $\left(\pi^{\prime}\right)^{-1}(p)=\psi_{0}\left(\pi^{-1}(p)\right) \subset Y$ is an irreducible and reduced rational curve in $Y$.

Now $\pi^{\prime}: E^{\prime} \rightarrow W$ is a well defined family of algebraic one-cycles on $Y$ over $W$ (see [15, Definition I.3.11 and Theorem I.3.17]), and induces a morphism $\iota: W \rightarrow \operatorname{Chow}(Y)$. Set $V:=\iota(W) \subset \operatorname{Chow}(Y)$. Then $V$ is a proper, covering family of irreducible and reduced rational curves on $Y$, so that $V$ is an unsplit family (see [15, Definition IV.2.1]).

The family $V$ induces an equivalence relation on $Y$ as a set, called $V$-equivalence; we refer the reader to $[12, \S 5]$ and references therein for the related definitions and properties.

We have $Z_{1} \cdot \psi_{0}(f)>0$; in particular $Z_{1}$ intersects every $V$-equivalence class in $Y$. This implies that

$$
\mathcal{N}_{1}(Y)=\mathbb{R}\left[\psi_{0}(f)\right]+\mathcal{N}_{1}\left(Z_{1}, Y\right)
$$

(see for instance [18, Lemma 3.2]). On the other hand by assumption $\mathcal{N}_{1}\left(Z_{1}, Y\right) \subsetneq \mathcal{N}_{1}(Y)$, therefore $\left[\psi_{0}(f)\right] \notin \mathcal{N}_{1}\left(Z_{1}, Y\right)$.

Let $T \subseteq Y$ be a $V$-equivalence class; notice that $T$ is either a closed subset, or a countable union of closed subsets. Let $T_{1} \subseteq T$ be an irreducible closed subset with $\operatorname{dim} T_{1}=\operatorname{dim} T$. We have $\mathcal{N}_{1}\left(T_{1}, Y\right)=\mathbb{R}\left[\psi_{0}(f)\right]$ by [15, Proposition IV.3.13.3], and $T_{1} \cap Z_{1} \neq \varnothing$. This implies that $\operatorname{dim}\left(T_{1} \cap Z_{1}\right)=0$ and $\operatorname{dim} T=\operatorname{dim} T_{1}=1$, that is: every $V$-equivalence class has dimension 1 . Then by [8, Proposition 1] there exists a contraction $\zeta: Y \rightarrow Y^{\prime}$ whose fibers coincide with $V$-equivalence classes.

Since $Y$ is smooth, $Y^{\prime}$ is irreducible, and $\zeta$ has connected fibers, the general fiber $l$ of $\zeta$ is irreducible and smooth. But $l$ is a $V$-equivalence class and $\operatorname{dim} l=1$, hence $l \cong \mathbb{P}^{1}$ and $-K_{Y} \cdot l=2$. Moreover $\mathrm{NE}(\zeta)=\mathbb{R}_{\geq 0}[l]$, so $-K_{Y}$ is $\zeta$-ample; this implies that $\zeta$ is an 
elementary contraction and a conic bundle, and that $Y^{\prime}$ is smooth (see [1, Theorem 3.1]). Finally $\zeta$ cannot have singular fibers, because the family $V$ is unsplit.

Let us carry on with the proof of Proposition 3.2.1. We have $\psi^{*}\left(Z_{1}\right) \cdot f_{0}=\left(E_{1}+\widehat{E}_{1}\right) \cdot f_{0}>0$, and $\mathcal{N}_{1}\left(Z_{1}, Y\right) \subseteq Z_{2}^{\perp} \subsetneq \mathcal{N}_{1}(Y)$ because $Z_{1} \cap Z_{2}=\varnothing$ (see Remark 3.1.2). Therefore we can apply Lemma 3.2.25 to $E_{0}$ and $\psi_{0}:=(\psi)_{\mid E_{0}}: E_{0} \rightarrow Y$. This shows that $\left[\psi\left(f_{0}\right)\right]$ belongs to an extremal ray of $Y$, whose contraction is a smooth conic bundle $\zeta: Y \rightarrow Y^{\prime}$.

We consider the composition $\psi^{\prime}:=\zeta \circ \psi: X \rightarrow Y^{\prime}$; the cone $\mathrm{NE}\left(\psi^{\prime}\right)$ is a $\left(c_{X}+1\right)$-dimensional face of $\mathrm{NE}(X)$ containing $R_{0}, R_{1}, \ldots, R_{c_{X}-1}, \widehat{R}_{1}, \ldots, \widehat{R}_{c_{X}-1}$, and $\rho_{Y^{\prime}}=\rho_{X}-c_{X}-1$.

Now we proceed similarly to the previous case. Let us consider the divisor

$$
H^{\prime}:=2 E_{0}+2 \sum_{i=1}^{c_{X}-1} E_{i}+\sum_{i=1}^{c_{X}-1} \widehat{E}_{i}
$$

on $X$. We have $H^{\prime} \cdot R_{0}>0, H^{\prime} \cdot R_{i}>0$ and $H^{\prime} \cdot \widehat{R}_{i}>0$ for every $i=1, \ldots, c_{X}-1$, and $\left(H^{\prime}\right)^{\perp} \supseteq L$. As before, $H^{\prime}$ is nef and defines a contraction onto a surface $\xi^{\prime}: X \rightarrow S$, such that $\xi^{\prime}\left(E_{0}\right), \xi^{\prime}\left(E_{i}\right)$, and $\xi^{\prime}\left(\widehat{E}_{i}\right)$ are irreducible rational curves and $E_{0}=\left(\xi^{\prime}\right)^{*}\left(\xi^{\prime}\left(E_{0}\right)\right)$, $E_{i}=\left(\xi^{\prime}\right)^{*}\left(\xi^{\prime}\left(E_{i}\right)\right), \widehat{E}_{i}=\left(\xi^{\prime}\right)^{*}\left(\xi^{\prime}\left(\widehat{E}_{i}\right)\right)$ for all $i=1, \ldots, c_{X}-1$.

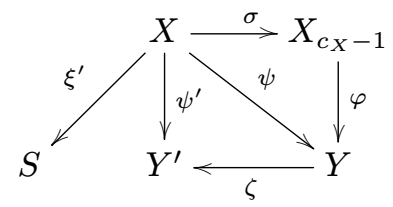

Then we consider the morphism $\pi^{\prime}: X \rightarrow S \times Y^{\prime}$ induced by $\xi^{\prime}$ and $\psi^{\prime}$. As in the previous case, one sees first that $\pi^{\prime}$ is finite, and then that it is an isomorphism, applying Remark 3.2.23. Finally we have $\rho_{S}=c_{X}+1$, because $\rho_{Y^{\prime}}=\rho_{X}-c_{X}-1$.

3.2.26. - We have shown in 3.2.21 and 3.2.24 that $X \cong S \times T$, where $S$ is a Del Pezzo surface with $\rho_{S}=c_{X}+1$ (and $T=Y$ in 3.2.21, while $T=Y^{\prime}$ in 3.2.24). In particular $c_{X} \leq 8$, as $\rho_{S} \leq 9$. Finally $c_{T} \leq c_{X}$ by Example 3.1, and this concludes the proof of Proposition 3.2.1.

\subsection{The case of codimension 3}

In this section we show the following.

Proposition 3.3.1. - Let $X$ be a Fano manifold with $c_{X}=3$. Then there exists a flat, quasi-elementary contraction $X \rightarrow T$ where $T$ is an $(n-2)$-dimensional Fano manifold, $\rho_{X}-\rho_{T}=4$, and $c_{T} \leq 3$. 
Proof. - By Corollary 3.2.2, there exist a prime divisor $D \subset X$ with $\operatorname{codim} \mathcal{N}_{1}(D, X)=3$, and a special Mori program for $-D$, such that $Q_{k} \not \subset \mathcal{N}_{1}\left(D_{k}, X_{k}\right)$.

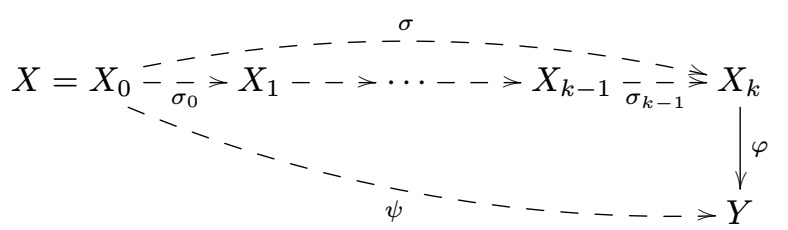

We apply Lemmas 2.7 and 2.8. By 2.7(2) and 2.7(3), there exist exactly two indices $i_{1}, i_{2} \in\{0, \ldots, k-1\}$ such that $Q_{i_{j}} \not \subset \mathcal{N}_{1}\left(D_{i_{j}}, X_{i_{j}}\right)$; the $\mathbb{P}^{1}$-bundles $E_{1}, E_{2} \subset X$ determined by the Mori program are the transforms of $\operatorname{Exc}\left(\sigma_{i_{1}}\right), \operatorname{Exc}\left(\sigma_{i_{2}}\right)$ respectively. Let moreover $\widehat{E}_{1}, \widehat{E}_{2} \subset X$ be as in 2.8(4). Recall that for $i=1,2 E_{i}$ (respectively, $\widehat{E}_{i}$ ) is a smooth $\mathbb{P}^{1}$-bundle with fiber $f_{i} \subset E_{i}$ (respectively, $\widehat{f_{i}} \subset \widehat{E}_{i}$ ), such that $E_{i} \cdot f_{i}=\widehat{E}_{i} \cdot \widehat{f}_{i}=-1$, $E_{i} \cdot \widehat{f}_{i}>0$, and $\widehat{E}_{i} \cdot f_{i}>0$. Moreover $\left(E_{1} \cup \widehat{E}_{1}\right) \cap\left(E_{2} \cup \widehat{E}_{2}\right)=\varnothing$.

3.3.3. - Before going on, let us give an outline of what we are going to do.

Our goal is to show that $k=2$ and $\sigma$ is just the composition of two smooth blow-ups with exceptional divisors $E_{1}$ and $E_{2}$. The proof of this fact is quite technical, and will be achieved in several steps.

We first show in 3.3.4 some properties of $\mathcal{N}_{1}\left(E_{i}, X\right)$ and $\mathcal{N}_{1}\left(\widehat{E}_{i}, X\right)$ which are needed in the sequel.

In 3.3.6 we prove that if $F \subset X$ is a prime divisor whose class in $\mathcal{N}^{1}(X)$ spans a onedimensional face of the cone of effective divisors $\operatorname{Eff}(X) \subset \mathcal{N}^{1}(X)$ (see 3.3.5), then $F$ must intersect both $E_{1} \cup \widehat{E}_{1}$ and $E_{2} \cup \widehat{E}_{2}$.

Then we show in 3.3 .7 that the Mori program (3.3.2) contains only two divisorial contractions, the ones with exceptional divisors $E_{1}$ and $E_{2}$. We proceed by contradiction, applying 3.3.6 to the exceptional divisor of a divisorial contraction (different from $\sigma_{i_{1}}$ and $\sigma_{i_{2}}$ ) in the Mori program.

In 3.3.9 and 3.3.10 we prove the existence of two disjoint prime divisors $F, \widehat{F} \subset X$, which are smooth $\mathbb{P}^{1}$-bundles with fibers $l \subset F, \widehat{l} \subset \widehat{F}$ such that $F \cdot l=\widehat{F} \cdot \widehat{l}=-1$, which are horizontal for the rational conic bundle $\psi: X \rightarrow Y$, and intersect the divisors $E_{1}, E_{2}, \widehat{E}_{1}, \widehat{E}_{2}$ in a suitable way.

Finally in 3.3.11 and 3.3.13 we use $F$ and $\widehat{F}$ to show that the Mori program (3.3.2) contains no flips. This means that $k=2, X_{2}$ and $Y$ are smooth, $\sigma$ is just a smooth blowup with exceptional divisors $E_{1}$ and $E_{2}$, and $\varphi$ and $\psi$ are conic bundles.

The situation is now analogous to the one in 3.2.24, and similarly to that case we prove that there is a smooth conic bundle $Y \rightarrow Y^{\prime}$, where $\operatorname{dim} Y^{\prime}=n-2$ (see 3.3.15). We have $\rho_{X}-\rho_{Y^{\prime}}=4$, and the contraction $X \rightarrow Y^{\prime}$ is flat and quasi-elementary.

To conclude, in 3.3.16 we show that the conic bundle $\varphi: X_{2} \rightarrow Y$ is smooth. This implies that every fiber of the conic bundle $\psi: X \rightarrow Y$ is reduced, and hence by a result in [21] both $Y$ and $Y^{\prime}$ are Fano. 
3.3.4. - For $i=1,2$ we have:

$\operatorname{codim} \mathcal{N}_{1}\left(E_{i}, X\right)=\operatorname{codim} \mathcal{N}_{1}\left(\widehat{E}_{i}, X\right)=3, \quad\left[\widehat{f}_{i}\right] \notin \mathcal{N}_{1}\left(E_{i}, X\right)$, and $\left[f_{i}\right] \notin \mathcal{N}_{1}\left(\widehat{E}_{i}, X\right) ;$ in particular $\mathcal{N}_{1}\left(E_{i}, X\right) \neq \mathcal{N}_{1}\left(\widehat{E}_{i}, X\right)$.

Indeed $\left[\widehat{f}_{i}\right] \notin \mathcal{N}_{1}\left(E_{i}, X\right)$ by $2.8(4)$. Moreover $D$ cannot contain any curve $\widehat{f}_{i}$, because $\sigma\left(\widehat{f}_{i}\right)$ is a fiber of $\varphi$, and $\varphi$ is finite on $D_{k} \subset X_{k}$. Therefore Lemma 3.1.10 yields the statement.

3.3.5. - Let $Z$ be a Mori dream space, and $\operatorname{Eff}(Z) \subset \mathcal{N}^{1}(Z)$ the convex cone spanned by classes of effective divisors. By [13, Proposition 1.11(2)] Eff $(Z)$ is a closed, convex polyhedral cone. If $F \subset Z$ is a prime divisor covered by a family of curves with which $F$ has negative intersection, then it is easy to see that $[F] \in \mathcal{N}^{1}(Z)$ spans a one-dimensional face of $\operatorname{Eff}(Z)$, and that the only prime divisor whose class belongs to this face is $F$ itself. In particular, this is true for $E_{1}, E_{2}, \widehat{E}_{1}, \widehat{E}_{2} \subset X$ (recall that $X$ is a Mori dream space by Theorem 2.1).

3.3.6. - Consider a prime divisor $F \subset X$ such that $[F]$ spans a one-dimensional face of $\operatorname{Eff}(X)$. We show that if $F$ is different from $E_{1}, E_{2}, \widehat{E}_{1}, \widehat{E}_{2}$, then $F$ must intersect both $E_{1} \cup \widehat{E}_{1}$ and $E_{2} \cup \widehat{E}_{2}$.

Indeed if for instance $F$ is disjoint from $E_{1} \cup \widehat{E}_{1}$, then

$$
\mathcal{N}_{1}\left(E_{1}, X\right) \cup \mathcal{N}_{1}\left(\widehat{E}_{1}, X\right) \subseteq E_{2}^{\perp} \cap \widehat{E}_{2}^{\perp} \cap F^{\perp}
$$

(see Remark 3.1.2). However this is impossible, because since $\left[E_{2}\right],\left[\widehat{E}_{2}\right],[F] \in \mathcal{N}^{1}(X)$ span three distinct one-dimensional faces of $\operatorname{Eff}(X)$, they must be linearly independent, thus $E_{2}^{\perp} \cap \widehat{E}_{2}^{\perp} \cap F^{\perp}$ has codimension 3, while $\mathcal{N}_{1}\left(E_{1}, X\right)$ and $\mathcal{N}_{1}\left(\widehat{E}_{1}, X\right)$ are distinct subspaces of codimension 3 by 3.3.4.

3.3.7. - Let us show that $\sigma_{i}$ is a flip for every $i \in\{0, \ldots, k-1\} \backslash\left\{i_{1}, i_{2}\right\}$, namely that $\sigma_{i_{1}}$ and $\sigma_{i_{2}}$ are the unique divisorial contractions in the Mori program (3.3.2).

By contradiction, suppose that there exists $i \in\{0, \ldots, k-1\} \backslash\left\{i_{1}, i_{2}\right\}$ such that $\sigma_{i}$ is a divisorial contraction. By 3.3.5 $\operatorname{Exc}\left(\sigma_{i}\right) \subset X_{i}$ is a prime divisor whose class spans a one-dimensional face of $\operatorname{Eff}\left(X_{i}\right)$, and it is the unique prime divisor in $X_{i}$ with class in $\mathbb{R}_{\geq 0}\left[\operatorname{Exc}\left(\sigma_{i}\right)\right] \cdot{ }^{(3)}$

Let $G \subset X$ be the transform of $\operatorname{Exc}\left(\sigma_{i}\right)$. By 2.8(3) and 2.8(4) there exists an open subset $U \subseteq X$, containing $E_{1}, E_{2}, \widehat{E}_{1}, \widehat{E}_{2}$, such that $\sigma$ is regular on $U$, and $\operatorname{Exc}\left(\sigma_{i}\right)$ is disjoint from the image of $U$ in $X_{i}$. Therefore $G \cap U=\varnothing$, in particular the divisor $G$ is disjoint from $E_{1}, E_{2}, \widehat{E}_{1}, \widehat{E}_{2}$.

Then 3.3.6 shows that $[G] \in \mathcal{N}^{1}(X)$ cannot span an extremal ray of $\operatorname{Eff}(X)$. This means that $[G]=\sum_{j} \lambda_{j}\left[G_{j}\right]$ with $\lambda_{j} \in \mathbb{R}_{>0}$ and $G_{j} \subset X$ prime divisors such that $[G] \notin \mathbb{R}_{\geq 0}\left[G_{j}\right]$; in particular $G_{j} \neq G$.

On the other hand, the map $\xi:=\sigma_{i-1} \circ \cdots \circ \sigma_{0}: X \rightarrow X_{i}$ induces a surjective linear $\operatorname{map} \xi_{*}: \mathcal{N}^{1}(X) \rightarrow \mathcal{N}^{1}\left(X_{i}\right)$ such that $\xi_{*}(\operatorname{Eff}(X))=\operatorname{Eff}\left(X_{i}\right)$. Then in $\mathcal{N}^{1}\left(X_{i}\right)$ we get

$$
\left[\operatorname{Exc}\left(\sigma_{i}\right)\right]=\left[\xi_{*}(G)\right]=\sum_{j} \lambda_{j}\left[\xi_{*}\left(G_{j}\right)\right]
$$

(3) Notice that $X_{i}$ is again a Mori dream space. 
hence $\left[\xi_{*}\left(G_{j}\right)\right] \in \mathbb{R}_{\geq 0}\left[\operatorname{Exc}\left(\sigma_{i}\right)\right]$ for every $j$. If $\xi_{*}\left(G_{j}\right) \neq 0$ for some $j$, then $\xi_{*}\left(G_{j}\right)$ is a prime divisor, and we get $\xi_{*}\left(G_{j}\right)=\operatorname{Exc}\left(\sigma_{i}\right)$ and hence $G_{j}=G$, a contradiction. Thus $\xi_{*}\left(G_{j}\right)=0$ for every $j$, therefore $\left[\operatorname{Exc}\left(\sigma_{i}\right)\right]=0$, again a contradiction.

3.3.8. - Let $F \subset X$ be a smooth prime divisor which is a $\mathbb{P}^{1}$-bundle with $F \cdot l=-1$, where $l \subset F$ is a fiber. Suppose that $F$ is different from $E_{1}, E_{2}, \widehat{E}_{1}, \widehat{E}_{2}$. Then:

- $F$ must intersect both $E_{1} \cup \widehat{E}_{1}$ and $E_{2} \cup \widehat{E}_{2}$;

- either $E_{1} \cdot l=\widehat{E}_{1} \cdot l=E_{2} \cdot l=\widehat{E}_{2} \cdot l=0$, or $\left(E_{1}+\widehat{E}_{1}\right) \cdot l>0$ and $\left(E_{2}+\widehat{E}_{2}\right) \cdot l>0$.

By 3.3.5 $[F]$ spans a one-dimensional face of $\operatorname{Eff}(X)$, so that 3.3.6 gives the first statement.

Recall that $\left(E_{1} \cup \widehat{E}_{1}\right) \cap\left(E_{2} \cup \widehat{E}_{2}\right)=\varnothing$. If $\left(E_{1}+\widehat{E}_{1}\right) \cdot l=0$, since $F$ intersects $E_{1} \cup \widehat{E}_{1}$, there exists a fiber $\bar{l}$ of the $\mathbb{P}^{1}$-bundle structure of $F$ which is contained in $E_{1} \cup \widehat{E}_{1}$. Thus $\bar{l} \cap\left(E_{2} \cup \widehat{E}_{2}\right)=\varnothing$, and we get $\left(E_{2}+\widehat{E}_{2}\right) \cdot l=0$. In this way we see that the intersections $\left(E_{1}+\widehat{E}_{1}\right) \cdot l,\left(E_{2}+\widehat{E}_{2}\right) \cdot l$ are either both zero or both positive, and this gives the second statement.

3.3.9. - We show that there exist two disjoint smooth prime divisors $F, \widehat{F} \subset X$, different from $E_{1}, E_{2}, \widehat{E}_{1}, \widehat{E}_{2}$, such that:

$-F$ and $\widehat{F}$ are $\mathbb{P}^{1}$-bundles, with fibers $l \subset F$ and $\widehat{l} \subset \widehat{F}$ respectively, such that $F \cdot l=\widehat{F} \cdot \widehat{l}=-1$; - the intersections $\left(E_{1}+\widehat{E}_{1}\right) \cdot l,\left(E_{1}+\widehat{E}_{1}\right) \cdot \widehat{l},\left(E_{2}+\widehat{E}_{2}\right) \cdot l,\left(E_{2}+\widehat{E}_{2}\right) \cdot \widehat{l}$ are all positive.

We have codim $\mathcal{N}_{1}\left(E_{1}, X\right)=3$ (see 3.3.4). Consider a special Mori program for $-E_{1}$ (which exists by Proposition 2.4), and let $G_{1}, \ldots, G_{s} \subset X$ be the $\mathbb{P}^{1}$-bundles determined by the Mori program. Recall from Lemma 2.7 that $G_{1}, \ldots, G_{s}$ are pairwise disjoint smooth prime divisors, with $2 \leq s \leq 3$, such that every $G_{i}$ is a $\mathbb{P}^{1}$-bundle with $G_{i} \cdot r_{i}=-1$, where $r_{i} \subset G_{i}$ is a fiber; moreover $E_{1} \cdot r_{i}>0$. In particular $G_{i} \neq E_{1}$ and $G_{i} \cap E_{1} \neq \varnothing$, thus $G_{i} \neq E_{2}$ and $G_{i} \neq \widehat{E}_{2}$. Finally, if $G_{i} \neq \widehat{E}_{1}$, by 3.3 .8 we have $\left(E_{1}+\widehat{E}_{1}\right) \cdot r_{i}>0$ and $\left(E_{2}+\widehat{E}_{2}\right) \cdot r_{i}>0$.

Suppose that $\left\{G_{1}, \ldots, G_{s}\right\}$ contains at least two divisors distinct from $\widehat{E}_{1}$, say $G_{1}$ and $G_{2}$. Then we set $F:=G_{1}$ and $\widehat{F}:=G_{2}$, and we are done.

Otherwise, we have $s=2$ and $G_{2}=\widehat{E}_{1}$. Then Lemma 2.8 applies, and by 2.8(4) there exists a smooth prime divisor $\widehat{G}_{2}$, having a $\mathbb{P}^{1}$-bundle structure with fiber $\widehat{r}_{2}$, such that:

$$
\widehat{G}_{2} \cdot \widehat{r}_{2}=-1, \quad G_{1} \cap \widehat{G}_{2}=\varnothing, \quad \widehat{G}_{2} \neq E_{1}, \quad \text { and } \widehat{E}_{1} \cdot \widehat{r}_{2}=1 .
$$

In particular $\widehat{G}_{2} \neq \widehat{E}_{1}$ and $\widehat{G}_{2} \cap \widehat{E}_{1} \neq \varnothing$, therefore $\widehat{G}_{2} \neq E_{2}$ and $\widehat{G}_{2} \neq \widehat{E}_{2}$. By 3.3.8 we have $\left(E_{1}+\widehat{E}_{1}\right) \cdot \widehat{r}_{2}>0$ and $\left(E_{2}+\widehat{E}_{2}\right) \cdot \widehat{r}_{2}>0$, thus we set $F:=G_{1}$ and $\widehat{F}:=\widehat{G}_{2}$.

3.3.10. - As soon as $F$ (respectively $\widehat{F}$ ) intersects one of the divisors $E_{i}$, then $F \cdot f_{i}>0$ and $E_{i} \cdot l>0$ (respectively $\widehat{F} \cdot f_{i}>0$ and $E_{i} \cdot \widehat{l}>0$ ), and similarly for $\widehat{E}_{i}$. In particular we have $F \cdot f>0$ and $\widehat{F} \cdot f>0$, where $f$ is a general fiber of $\psi$.

Suppose for instance that $F \cap E_{1} \neq \varnothing$. If $E_{1} \cdot l=0$, then $E_{1}$ contains some curve $l$, but this is impossible because $\left(E_{2}+\widehat{E}_{2}\right) \cdot l>0$ while $E_{1} \cap\left(E_{2} \cup \widehat{E}_{2}\right)=\varnothing$; thus $E_{1} \cdot l>0$.

If $F \cdot f_{1}=0$, then $F$ contains an irreducible curve $\bar{f}_{1}$ which is a fiber of the $\mathbb{P}^{1}$-bundle structure on $E_{1}$. Let $\pi: F \rightarrow G$ be the $\mathbb{P}^{1}$-bundle structure on $F$, and $\pi_{*}: \mathcal{N}_{1}(F) \rightarrow \mathcal{N}_{1}(G)$ the push-forward. Notice that $\pi\left(\bar{f}_{1}\right)$ is a curve, because $\bar{f}_{1}$ and $l$ are not numerically equivalent in $X$, and hence neither in $F$. 
Consider the surface $S:=\pi^{-1}\left(\pi\left(\bar{f}_{1}\right)\right)$. Then $\pi_{*}\left(\mathcal{N}_{1}(S, F)\right)=\mathbb{R} \pi_{*}\left(\left[\bar{f}_{1}\right]_{F}\right)$, hence $\mathcal{N}_{1}(S, F)=\operatorname{ker} \pi_{*} \oplus \mathbb{R}\left[\bar{f}_{1}\right]_{F}=\mathbb{R}[l]_{F} \oplus \mathbb{R}\left[\bar{f}_{1}\right]_{F}$, and $\mathcal{N}_{1}(S, X)=\mathbb{R}[l] \oplus \mathbb{R}\left[f_{1}\right]$.

Since $\widehat{E}_{1} \cdot \bar{f}_{1}>0$, we have $S \cap \widehat{E}_{1} \neq \varnothing$, and there exists an irreducible curve $C \subset S \cap \widehat{E}_{1}$. Thus $[C] \in \mathcal{N}_{1}(S, X)$, so that $C \equiv \lambda l+\mu f_{1}$ with $\lambda, \mu \in \mathbb{R}$. On the other hand $C \cap\left(E_{2} \cup \widehat{E}_{2}\right)=\varnothing$ (because $C \subset \widehat{E}_{1}$ ) and

$$
0=\left(E_{2}+\widehat{E}_{2}\right) \cdot C=\lambda\left(E_{2}+\widehat{E}_{2}\right) \cdot l,
$$

which by 3.3.9 yields $\lambda=0, \mu \neq 0$ and $\left[f_{1}\right]=(1 / \mu)[C] \in \mathcal{N}_{1}\left(\widehat{E}_{1}, X\right)$, a contradiction with 3.3.4.

Therefore $F \cdot f_{1}>0$. We have $f \equiv f_{1}+\widehat{f}_{1}$ (see 2.8(4)), and $F \cdot \widehat{f}_{1} \geq 0$ because $F \neq \widehat{E}_{1}$ (see 3.3.9), hence $F \cdot f>0$.

3.3.11. - For every $i \in\{0, \ldots, k\}$ let $F_{i}, \widehat{F}_{i} \subset X_{i}$ be the transforms of $F, \widehat{F}$. Let us show that for any $i \in\{0, \ldots, k-1\} \backslash\left\{i_{1}, i_{2}\right\}$, the divisors $F_{i}$ and $\widehat{F}_{i}$ are disjoint from $\operatorname{Locus}\left(Q_{i}\right)$.

By contradiction, suppose for instance that this is not true for $F$, and let $j \in\{0, \ldots, k-1\} \backslash\left\{i_{1}, i_{2}\right\}$ be the smallest index such that $F_{j}$ intersects $\operatorname{Locus}\left(Q_{j}\right)$. Recall from 3.3.7 that $\sigma_{i}$ is a flip for every $i \in\{0, \ldots, k-1\} \backslash\left\{i_{1}, i_{2}\right\}$; in particular, $Q_{j}$ is a small extremal ray, and $\sigma_{j}$ is a flip.

After 2.8(3), $\sigma$ is regular on the divisors $E_{1}, E_{2}, \widehat{E}_{1}, \widehat{E}_{2}$, and $\operatorname{Locus}\left(Q_{j}\right)$ is disjoint from their images in $X_{j}$.

Recall from 2.6(4) the definition of $A_{i} \subset X_{i}$, for $i \in\{1, \ldots, k\}: A_{1} \subset X_{1}$ is the indeterminacy locus of $\sigma_{0}^{-1}$, and for $i \geq 2$, if $\sigma_{i-1}$ is a divisorial contraction (respectively, if $\sigma_{i-1}$ is a flip), $A_{i}$ is the union of $\sigma_{i-1}\left(A_{i-1}\right)$ (respectively, the transform of $A_{i-1}$ ) and the indeterminacy locus of $\sigma_{i-1}^{-1}$.

If $j>0$, by the minimality of $j, F_{j}$ does not intersect the loci of the previous flips, hence it can intersect $A_{j}$ only along the images of $E_{1}$ and $E_{2}$. Therefore

$$
\operatorname{Locus}\left(Q_{j}\right) \cap F_{j} \cap A_{j}=\varnothing .
$$

Let $\alpha_{j}: X_{j} \rightarrow Y_{j}$ be the contraction of $Q_{j}$. Suppose first that $\alpha_{j}$ is finite on $F_{j}$. Then $\operatorname{Locus}\left(Q_{j}\right)=\operatorname{Exc}\left(\alpha_{j}\right) \not \subset F_{j}$, and since $F_{j} \cap \operatorname{Locus}\left(Q_{j}\right) \neq \varnothing$, we have $F_{j} \cdot Q_{j}>0$. Hence every non trivial fiber of $\alpha_{j}$ must have dimension 1, otherwise $\alpha_{j}$ would not be finite on $F_{j}$.

If $j=0$, then $\alpha_{0}$ is a small contraction of a smooth Fano variety with one-dimensional fibers, which is impossible, see [3, Theorem 4.1].

Suppose that $j>0$. If $C_{0} \subset X_{j}$ is an irreducible curve in a fiber of $\alpha_{j}$, then $C_{0}$ must intersect $F_{j}$, hence $C_{0} \nsubseteq A_{j}$ by (3.3.12); in particular $C_{0} \nsubseteq \operatorname{Sing}\left(X_{j}\right)$ (recall that $\operatorname{Sing}\left(X_{j}\right) \subseteq A_{j}$ by 2.6(4)). Then [14, Lemma 1] yields $-K_{X_{j}} \cdot C_{0} \leq 1$, and [11, Lemma 3.8] implies that $C_{0} \cap A_{j}=\varnothing$. We conclude that $\operatorname{Locus}\left(Q_{j}\right) \subseteq X_{j} \backslash A_{j}$, and this is again impossible by [3, Theorem 4.1], because $-K_{X_{j}} \cdot Q_{j}>0$ and $\left(\alpha_{j}\right)_{\mid X_{j} \backslash A_{j}}: X_{j} \backslash A_{j} \rightarrow Y_{j} \backslash \alpha_{j}\left(A_{j}\right)$ is a small contraction of a smooth variety with one-dimensional fibers.

Suppose now that $\alpha_{j}$ is not finite on $F_{j}$. Then there exists an irreducible curve $C_{1} \subset F_{j}$ with $\left[C_{1}\right] \in Q_{j}$; in particular $C_{1}$ is disjoint from the images of $E_{1}, E_{2}, \widehat{E}_{1}, \widehat{E}_{2}$ in $X_{j}$. Consider the transform $\widetilde{C}_{1} \subset F \subset X$ of $C_{1}$, so that $\widetilde{C}_{1}$ is disjoint from $E_{1}, E_{2}, \widehat{E}_{1}, \widehat{E}_{2}$. 
Recall that $F$ intersects both $E_{1} \cup \widehat{E}_{1}$ and $E_{2} \cup \widehat{E}_{2}$ by 3.3.8. We assume that $F$ intersects $E_{1}$ and $E_{2}$, the other cases being analogous. Then $E_{1} \cdot l>0$ by 3.3.10, so that using 3.1.3(3) we get

$$
\widetilde{C}_{1} \equiv \lambda l+\mu C_{2},
$$

where $C_{2} \subset F \cap E_{1}$ is a curve, $\lambda, \mu \in \mathbb{R}$, and $\mu \geq 0$. In particular $C_{2} \cap E_{2}=\varnothing$, therefore $0=E_{2} \cdot \widetilde{C}_{1}=\lambda E_{2} \cdot l$. On the other hand $E_{2} \cdot l>0$ by 3.3.10, and this implies that $\lambda=0$ and $\widetilde{C}_{1} \equiv \mu C_{2}$. Recall that the map $X \rightarrow X_{j}$ is regular on $F$ by the minimality of $j$, and call $C_{2}^{\prime}$ the image of $C_{2}$ in $X_{j}$. We deduce that $C_{1} \equiv \mu C_{2}^{\prime}$ in $X_{j}$, so that $\left[C_{2}^{\prime}\right] \in Q_{j}$. But $C_{2}^{\prime}$ is contained in the image of $E_{1}$, which is disjoint from $\operatorname{Locus}\left(Q_{j}\right)$, and we have a contradiction.

3.3.13. - We show that $k=2$ in (3.3.2), so that $i_{1}=0$ and $i_{2}=1$.

By contradiction, suppose that $k>2$. Recall from 3.3.7 that $\sigma_{i}$ is a flip for every $i \in\{0, \ldots, k-1\} \backslash\left\{i_{1}, i_{2}\right\}$, so equivalently we are assuming that the Mori program (3.3.2) contains some flips.

We define an integer $m \in\{k-3, k-2, k-1\}$ and a morphism $\eta: X_{m+1} \rightarrow X_{k}$ as follows:

- if $\sigma_{k-1}$ is a flip, set $m:=k-1$ and $\eta:=\operatorname{Id}_{X_{k}}$;

- if $\sigma_{k-1}$ is a contraction and $\sigma_{k-2}$ is a flip, set $m:=k-2$ and $\eta:=\sigma_{k-1}: X_{k-1} \rightarrow X_{k}$;

- if both $\sigma_{k-1}$ and $\sigma_{k-2}$ are contractions, set $m:=k-3$ and $\eta:=\sigma_{k-2} \circ \sigma_{k-1}: X_{k-2} \rightarrow X_{k}$.

It follows from these definitions that $Q_{m}$ is a small extremal ray of $X_{m}$ and $\sigma_{m}: X_{m} \rightarrow X_{m+1}$ is a flip; let $Q_{m+1}^{\prime}$ be the corresponding small extremal ray of $X_{m+1}$. Set moreover $\tilde{\varphi}:=\varphi \circ \eta: X_{m+1} \rightarrow Y$.

$$
X \cong ニ ニ X_{m}-\bar{\sigma}_{m}>X_{m+1} \stackrel{-}{\longrightarrow} X_{k}
$$

We keep the same notations as in the proof of Lemma 2.8; in particular we set $T_{i}:=\sigma\left(E_{i}\right) \subset X_{k}$ for $i=1,2$. Notice that when $m=k-2$ (respectively, $m=k-3$ ), $\eta$ is just the smooth blow-up of $T_{2} \subset X_{k}$ (respectively, of $T_{1} \cup T_{2} \subset X_{k}$ ).

Recall that for $j=1,2$ we have $Q_{i_{j}} \not \subset \mathcal{N}_{1}\left(D_{i_{j}}, X_{i_{j}}\right)$, in particular $\sigma_{i_{j}}$ is finite on $D_{i_{j}}$; this implies that $\eta$ is finite on $D_{m+1}$.

We show that every fiber of $\widetilde{\varphi}$ has dimension 1 . Indeed this is true for $\varphi$ by 2.8(1). Moreover $\eta$ is an isomorphism over $X_{k} \backslash\left(T_{1} \cup T_{2}\right)$, therefore $\widetilde{\varphi}$ has one-dimensional fibers over $Y \backslash \varphi\left(T_{1} \cup T_{2}\right)$. On the other hand, we know by 2.8(3) that there exist open subsets $U \subseteq X$ and $V \subseteq Y$ such that $\varphi\left(T_{1} \cup T_{2}\right) \subset V$, both $\psi: U \rightarrow V$ and $\varphi_{\mid \varphi^{-1}(V)}: \varphi^{-1}(V) \rightarrow V$ are conic bundles, and $\sigma_{\mid U}: U \rightarrow \varphi^{-1}(V)$ is just the blow-up of $T_{1}$ and $T_{2}$. This implies that $\widetilde{\varphi}_{\mid \widetilde{\varphi}^{-1}(V)}: \widetilde{\varphi}^{-1}(V) \rightarrow V$ is a conic bundle, in particular it has one-dimensional fibers over $\varphi\left(T_{1} \cup T_{2}\right) \subset V$.

Recall from 2.8(1) that $\varphi$ is finite on $D_{k}=\eta\left(D_{m+1}\right)$, and since $\eta$ is finite on $D_{m+1}$, we deduce that $\widetilde{\varphi}$ must be finite on $D_{m+1}$. Notice also that $D_{m+1} \supset A_{m+1} \supseteq \operatorname{Locus}\left(Q_{m+1}^{\prime}\right)$ (see 2.6(4)). As in the proof of Lemma 2.8, using [11, Lemma 3.8] we see that every fiber of $\widetilde{\varphi}$ which intersects $\operatorname{Locus}\left(Q_{m+1}^{\prime}\right)$ is an integral rational curve. 
Let $C \subset X_{m+1}$ be an irreducible curve with $[C] \in Q_{m+1}^{\prime}$, and set $S:=\widetilde{\varphi}^{-1}(\widetilde{\varphi}(C))$, so that $S$ is an irreducible surface.

Since, by 3.3.10, $F$ and $\widehat{F}$ have positive intersection with a general fiber of $\psi$ in $X, F_{m+1}$ and $\widehat{F}_{m+1}$ have positive intersection with every fiber of $\widetilde{\varphi}$ in $X_{m+1}$. In particular, $F_{m+1}$ and $\widehat{F}_{m+1}$ intersect $S$.

On the other hand by 3.3.11 the divisors $F_{m}$ and $\widehat{F}_{m}$ in $X_{m}$ are disjoint from Locus $\left(Q_{m}\right)$, therefore $F_{m+1}$ and $\widehat{F}_{m+1}$ are disjoint from $\operatorname{Locus}\left(Q_{m+1}^{\prime}\right)$. We deduce that:

$$
F_{m+1} \cap C=\widehat{F}_{m+1} \cap C=\varnothing \text { and } \operatorname{dim}\left(F_{m+1} \cap S\right)=\operatorname{dim}\left(\widehat{F}_{m+1} \cap S\right)=1 .
$$

For $i=1,2$ call $G_{i}$ the image of $E_{i}$ in $X_{m+1}$, so that $T_{i}=\eta\left(G_{i}\right)$ and $\varphi\left(T_{i}\right)=\widetilde{\varphi}\left(G_{i}\right)$. Notice that $A_{k} \backslash\left(T_{1} \cup T_{2}\right)=\eta\left(A_{m+1} \backslash\left(G_{1} \cup G_{2}\right)\right)$.

Recall that the open subset $V \subseteq Y$ was defined in (2.10) as

$$
V:=Y \backslash \varphi\left(A_{k} \backslash\left(T_{1} \cup T_{2}\right)\right)=Y \backslash \widetilde{\varphi}\left(A_{m+1} \backslash\left(G_{1} \cup G_{2}\right)\right) .
$$

By 2.7(1) and 2.8(2) we have $\operatorname{Locus}\left(Q_{m+1}^{\prime}\right) \cap\left(G_{1} \cup G_{2}\right)=\varnothing$. In particular $C \subseteq \operatorname{Locus}\left(Q_{m+1}^{\prime}\right) \subseteq A_{m+1} \backslash\left(G_{1} \cup G_{2}\right)$, thus

$$
\widetilde{\varphi}(C) \subseteq Y \backslash V .
$$

On the other hand we also have $\widetilde{\varphi}\left(G_{1} \cup G_{2}\right)=\varphi\left(T_{1} \cup T_{2}\right) \subset V$, therefore we deduce that $\widetilde{\varphi}\left(G_{1} \cup G_{2}\right) \cap \widetilde{\varphi}(C)=\varnothing$ and hence

$$
\left(G_{1} \cup G_{2}\right) \cap S=\varnothing .
$$

Finally by 3.3.9 we have $F \cap \widehat{F}=\varnothing$ in $X$, and by 3.3.11 the divisors $F$ and $\widehat{F}$ are disjoint from the locus of every flip in the Mori program (3.3.2). This implies that $F_{m+1} \cap \widehat{F}_{m+1} \subseteq G_{1} \cup G_{2}$, therefore:

$$
F_{m+1} \cap \widehat{F}_{m+1} \cap S=\varnothing .
$$

Together with (3.3.14), this yields that $C, F_{m+1} \cap S$, and $\widehat{F}_{m+1} \cap S$ are pairwise disjoint curves in $S$.

Let $C^{\prime}$ be an irreducible component of $\widehat{F}_{m+1} \cap S$. Since $\widetilde{\varphi}_{\mid S}: S \rightarrow \widetilde{\varphi}(C)$ is a fibration in integral rational curves, we have $C^{\prime} \equiv \lambda C+\mu f$ where $\lambda, \mu \in \mathbb{R}$ and $f \subset S$ is a fiber. Then $0=F_{m+1} \cdot C^{\prime}=\mu F_{m+1} \cdot f$ while $F_{m+1} \cdot f>0$, hence $\mu=0$ and $\left[C^{\prime}\right] \in Q_{m+1}^{\prime}$. Therefore $C^{\prime} \subseteq \operatorname{Locus}\left(Q_{m+1}^{\prime}\right) \cap F_{m+1}$, a contradiction because $\operatorname{Locus}\left(Q_{m+1}^{\prime}\right) \cap F_{m+1}=\varnothing$.

3.3.15. - Since $k=2, X_{2}$ is smooth and $\sigma: X \rightarrow X_{2}$ is just the blow-up of two disjoint smooth subvarieties $T_{1}, T_{2} \subset X_{2}$, of codimension 2. In fact, we have $A_{2}=T_{1} \cup T_{2}$ (see 2.6(4)), and by (2.10) the description in 2.8(3) and 2.8(4) holds with $V=Y$ and $U=X$. In particular, $Y$ is smooth, $\varphi: X_{2} \rightarrow Y$ and $\psi: X \rightarrow Y$ are conic bundles, $\rho_{X}-\rho_{Y}=3$, and the divisors $Z_{1}=\psi\left(E_{1}\right)$ and $Z_{2}=\psi\left(E_{2}\right)$ are disjoint in $Y$. Moreover we have $\psi(F)=Y$ by 3.3.10.

The situation is very similar to the case where $\varphi$ is finite on $\left(E_{0}\right)_{s}$ in 3.2.24, with the difference that the $E_{i}$ 's do not need to be products. In the same way we use Lemma 3.2.25 to show that $[\psi(l)] \in \mathrm{NE}(Y)$ belongs to an extremal ray of $Y$, whose contraction is a smooth conic bundle $\zeta: Y \rightarrow Y^{\prime}$, finite on $Z_{1}$ and $Z_{2}$; in particular $Y^{\prime}$ is smooth of dimension $n-2$. The contraction $\psi^{\prime}:=\zeta \circ \psi: X \rightarrow Y^{\prime}$ is equidimensional and hence flat, and $\rho_{X}-\rho_{Y^{\prime}}=4$. 
Moreover the general fiber of $\psi^{\prime}$ is a Del Pezzo surface $S$ containing curves $f_{1}, \widehat{f}_{1}, f_{2}, \widehat{f}_{2}, l$, hence $\mathcal{N}_{1}(S, X)=\operatorname{ker}\left(\psi^{\prime}\right)_{*}$ and $\psi^{\prime}$ is quasi-elementary.

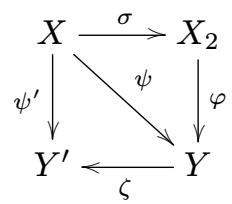

3.3.16. - We show that the conic bundle $\varphi: X_{2} \rightarrow Y$ is smooth.

By contradiction, suppose that this is not the case, and let $\Delta_{\varphi} \subset Y$ be the discriminant divisor of $\varphi$. Recall that this is an effective, reduced divisor in $Y$ such that $\varphi^{-1}(y)$ is singular if and only if $y \in \Delta_{\varphi}$.

Consider also the discriminant divisor $\Delta_{\psi} \subset Y$ of the conic bundle $\psi: X \rightarrow Y$. Since $\varphi$ is smooth over $Z_{1}$ and $Z_{2}$, the divisors $\Delta_{\varphi}, Z_{1}, Z_{2}$ are pairwise disjoint, and $\Delta_{\psi}=\Delta_{\varphi} \cup Z_{1} \cup Z_{2}$.

The fibers of $\psi$ over $Z_{1} \cup Z_{2}$ are singular but reduced, hence $\psi^{-1}(y)$ is non-reduced if and only if $\varphi^{-1}(y)$ is. Let $W \subset \Delta_{\varphi}$ be the set of points $y$ such that $\psi^{-1}(y)$ (equivalently, $\varphi^{-1}(y)$ ) is non-reduced. Then $W$ is a closed subset of $Y$, and $W \subseteq \operatorname{Sing}\left(\Delta_{\varphi}\right)$ (see for instance [19, Proposition 1.8(5.c)]). Moreover by [21, Proposition 4.3] we know that $-K_{Y} \cdot C>0$ for every irreducible curve $C \subset Y$ not contained in $W$.

For $i=1,2$ we have $\operatorname{codim} \mathcal{N}_{1}\left(Z_{i}, Y\right) \leq 1$, because $\zeta\left(Z_{i}\right)=Y^{\prime}$ and hence $\zeta_{*}\left(\mathcal{N}_{1}\left(Z_{i}, Y\right)\right)=\mathcal{N}_{1}\left(Y^{\prime}\right)$. This yields $Z_{1}^{\perp}=Z_{2}^{\perp}=\Delta_{\varphi}^{\perp}=\mathcal{N}_{1}\left(Z_{1}, Y\right)=\mathcal{N}_{1}\left(Z_{2}, Y\right)$ (see Remark 3.1.2). The three divisors $\Delta_{\varphi}, Z_{1}, Z_{2}$ are numerically proportional, nef, and cut a facet of $\operatorname{NE}(Y)$, whose contraction $\beta: Y \rightarrow \mathbb{P}^{1}$ sends $\Delta_{\varphi}, Z_{1}, Z_{2}$ to points (see [10, Lemma 2.6]). Even if a priori we do not know whether every curve contracted by $\beta$ has positive anticanonical degree, the general fiber of $\beta$ does not meet $W$, therefore it is a Fano manifold. Moreover $\mathrm{NE}(\beta)$ is generated by finitely many classes of rational curves (see [10, Lemma 2.6]). Thus the same proof as [11, Lemma 4.9] yields that $Y \cong \mathbb{P}^{1} \times Y^{\prime}$, and $\Delta_{\varphi}=\{p t s\} \times Y^{\prime}$.

In particular $\Delta_{\varphi}$ is smooth, hence $W=\varnothing$ and $Y$ is Fano. Because $Y \cong \mathbb{P}^{1} \times Y^{\prime}, Y^{\prime}$ is Fano too, so that each connected component of $\Delta_{\varphi}$ is simply connected. However this is impossible, because by a standard construction the conic bundle $\varphi$ defines a double cover of every irreducible component of $\Delta_{\varphi}$, obtained by considering the components of the fibers in the appropriate Hilbert scheme of lines, see [4, §1.5] and [19, §1.17]. Since $\varphi$ is an elementary contraction, this double cover is non-trivial; on the other hand it is also étale, because every fiber of $\varphi$ is reduced, and we have a contradiction.

3.3.17. - Since $\varphi: X_{2} \rightarrow Y$ is smooth, every fiber of the conic bundle $\psi: X \rightarrow Y$ is reduced. Then [21, Proposition 4.3] shows that $Y$ and $Y^{\prime}$ are Fano. Finally $c_{Y^{\prime}} \leq 3$ by the following remark, which concludes the proof of Proposition 3.3.1.

Remark 3.3.18. - Let $X$ be a Fano manifold, $\varphi: X \rightarrow Y$ a surjective morphism, and $D \subset X \quad$ a prime divisor. We have $\mathcal{N}_{1}(\varphi(D), Y)=\varphi_{*}\left(\mathcal{N}_{1}(D, X)\right)$, hence $\operatorname{codim} \mathcal{N}_{1}(D, X) \geq \operatorname{codim} \mathcal{N}_{1}(\varphi(D), Y)$. In particular, if $Y$ is a Fano manifold, then $c_{Y} \leq c_{X}$. 


\section{Applications}

In this final section we prove the results stated in the introduction.

Proof of Theorem 1.1. - We have $c_{X} \geq \operatorname{codim} \mathcal{N}_{1}(D, X) \geq 3$. If $c_{X}=3$, Theorem 3.3 yields (ii). If instead $c_{X} \geq 4$, applying iteratively Theorem 3.3, we can write $X=S_{1} \times \cdots \times S_{r} \times Z$, where $S_{i}$ are Del Pezzo surfaces, $r \geq 1$, and $Z$ is a Fano manifold with $c_{Z} \leq 3$.

If $D$ dominates $Z$ under the projection, up to reordering $S_{1}, \ldots, S_{r}$ we can assume that $D$ dominates $S_{2} \times \cdots \times S_{r} \times Z$. Then $\operatorname{codim} \mathcal{N}_{1}(D, X) \leq \rho_{S_{1}}-1$ (see Example 3.1), and we get (i).

Suppose instead that $D=S_{1} \times \cdots \times S_{r} \times D_{Z}$, where $D_{Z} \subset Z$ is a prime divisor. Then

$$
3 \geq c_{Z} \geq \operatorname{codim} \mathcal{N}_{1}\left(D_{Z}, Z\right)=\operatorname{codim} \mathcal{N}_{1}(D, X) \geq 3
$$

and the inequalities above are equalities. Therefore again by Theorem 3.3 we have a flat, quasi-elementary contraction $Z \rightarrow W$, where $W$ is a Fano manifold with $\operatorname{dim} W=\operatorname{dim} Z-2$, and $\rho_{Z}-\rho_{W}=4$. Then the induced contraction $X \rightarrow S_{1} \times \cdots \times S_{r} \times W$ satisfies (ii).

Proof of Corollary 1.3. - We have $c_{X} \geq \operatorname{codim} \mathcal{N}_{1}(D, X) \geq 3$. Suppose that $X$ is not a product of a Del Pezzo surface with another variety. Then Theorem 3.3 shows that $c_{X}=3$ and there is a quasi-elementary contraction $X \rightarrow T$ where $T$ is a Fano manifold, $\operatorname{dim} T=n-2$, and $\rho_{X}-\rho_{T}=4$. If $n=4$, [10, Theorem 1.1] implies that $\rho_{T} \leq 2$, hence $\rho_{X} \leq 6$. The case $n=5$ follows similarly.

Lemma 4.1. - Let $X$ be a Fano manifold, $D \subset X$ a prime divisor, and $\varphi: X \rightarrow Y a$ contraction. Then $\operatorname{codim} \mathcal{N}_{1}(\varphi(D), Y) \leq 8$.

Suppose moreover that codim $\mathcal{N}_{1}(\varphi(D), Y) \geq 4$. Then $X \cong S \times T$ and $Y \cong W \times Z$, where $S$ is a Del Pezzo surface, $W$ is a blow-down of $S$, and codim $\varphi(D) \leq 2$. More precisely, one of the following holds:

(i) $\varphi(D)$ is a divisor in $Y$, and dominates $Z$ under the projection;

(ii) $\varphi(D)=\{p\} \times Z$ and $D=C \times T$, where $C \subset S$ is a curve contracted to $p \in W$.

Proof. - We have codim $\mathcal{N}_{1}(\varphi(D), Y) \leq \operatorname{codim} \mathcal{N}_{1}(D, X) \leq 8$ by Remark 3.3.18 and Theorem 1.1. Suppose that $\operatorname{codim} \mathcal{N}_{1}(\varphi(D), Y) \geq 4$. Then, again by Theorem 1.1, $X \cong S \times T$ where $S$ is a Del Pezzo surface, and $D$ dominates $T$ under the projection. Therefore $Y \cong W \times Z, \varphi$ is induced by two contractions $S \rightarrow W$ and $f: T \rightarrow Z$, and $\varphi(D)$ dominates $Z$ under the projection.

In particular $\operatorname{dim} W \leq 2$ and $\operatorname{dim} \mathcal{N}_{1}(\varphi(D), Y) \geq \rho_{Z}$, hence $\rho_{W} \geq \operatorname{codim} \mathcal{N}_{1}(\varphi(D), Y) \geq 4$. This implies that $\operatorname{dim} W=2$, thus $W$ is a blow-down of $S$, and $\varphi(D)$ has codimension 1 or 2 in $Y$.

If $\varphi(D)$ is a divisor, we have (i). Suppose that $\operatorname{codim} \varphi(D)=2$, and consider the factorization of $\varphi$ as $S \times T \stackrel{\psi}{\rightarrow} W \times T \stackrel{\xi}{\rightarrow} W \times Z$. Then $\xi=\left(\operatorname{Id}_{W}, f\right)$ induces an isomorphism $W \times\{t\} \rightarrow W \times\{f(t)\}$ for every $t \in T$. If $t$ is general, we have $\operatorname{dim} \varphi(D) \cap(W \times\{f(t)\})=0$ and $\psi(D) \cap(W \times\{t\}) \cong \varphi(D) \cap(W \times\{f(t)\})$. This implies that $\psi(D)$ has codimension 2 in $W \times T$, hence $D$ is an exceptional divisor of $\psi$, which gives the statement. 
Corollary 1.4 follows from Theorem 1.1, while Corollary 1.5 is a straightforward application of Lemma 4.1 (just take the Stein factorization of $\varphi$ ).

Proof of Corollary 1.6. - By taking the Stein factorization, we can assume that $\varphi$ is a contraction; we also assume that $\rho_{Y} \geq 6$. By [10, Lemma 2.6] we know that $Y$ has some elementary contraction $\psi: Y \rightarrow Z$, and $\operatorname{dim} Z \geq 2$ because $\rho_{Z} \geq 5$.

We define a prime divisor $D \subset X$ depending on $\psi$, as follows. If $\operatorname{dim} Z=2$, let $D \subset X$ be any prime divisor such that $\operatorname{dim} \psi(\varphi(D))=1$. If $\psi$ is birational and divisorial, let $D \subset X$ be a prime divisor such that $\varphi(D) \subseteq \operatorname{Exc}(\psi)$. If $\psi$ is birational and small, its lifting in $X$ (see $[10, \S 2.5])$ must be an elementary contraction of type $(n-1, n-2)^{s m}$; let $D$ be its exceptional divisor: then again we have $\varphi(D) \subseteq \operatorname{Exc}(\psi)$.

In any case $\operatorname{dim} \mathcal{N}_{1}(\varphi(D), Y) \leq 2$, so that Lemma 4.1 implies the statement.

\section{REFERENCES}

[1] T. Ando, On extremal rays of the higher-dimensional varieties, Invent. Math. 81 (1985), 347-357.

[2] M. Andreatta, E. Ballico, J. A. Wiśniewski, Vector bundles and adjunction, Internat. J. Math. 3 (1992), 331-340.

[3] M. Andreatta, J. A. Wiśniewski, A view on contractions of higher-dimensional varieties, in Algebraic geometry-Santa Cruz 1995, Proc. Sympos. Pure Math. 62, Amer. Math. Soc., 1997, 153-183.

[4] A. Beauville, Prym varieties and the Schottky problem, Invent. Math. 41 (1977), 149196.

[5] C. Birkar, P. Cascini, C. D. Hacon, J. McKernan, Existence of minimal models for varieties of log general type, J. Amer. Math. Soc. 23 (2010), 405-468.

[6] L. Bonavero, F. Campana, J. A. Wiśniewski, Variétés complexes dont l'éclatée en un point est de Fano, C. R. Math. Acad. Sci. Paris 334 (2002), 463-468.

[7] L. Bonavero, C. Casagrande, O. Debarre, S. Druel, Sur une conjecture de Mukai, Comment. Math. Helv. 78 (2003), 601-626.

[8] L. Bonavero, C. Casagrande, S. Druel, On covering and quasi-unsplit families of curves, J. Eur. Math. Soc. (JEMS) 9 (2007), 45-57.

[9] C. Casagrande, Toric Fano varieties and birational morphisms, Int. Math. Res. Not. 27 (2003), 1473-1505.

[10] C. Casagrande, Quasi-elementary contractions of Fano manifolds, Compos. Math. 144 (2008), 1429-1460.

[11] C. Casagrande, On Fano manifolds with a birational contraction sending a divisor to a curve, Michigan Math. J. 58 (2009), 783-805.

[12] O. Debarre, Higher-dimensional algebraic geometry, Universitext, Springer, 2001.

[13] Y. Hu, S. Keel, Mori dream spaces and GIT, Michigan Math. J. 48 (2000), 331-348.

[14] S. IsHII, Quasi-Gorenstein Fano 3-folds with isolated nonrational loci, Compositio Math. 77 (1991), 335-341.

$4^{\mathrm{e}}$ SÉRIE - TOME $45-2012-\mathrm{N}^{\circ} 3$ 
[15] J. Kollár, Rational curves on algebraic varieties, Ergebn. Math. Grenzg. 32, Springer, 1996.

[16] J. Kollár, S. Mori, Birational geometry of algebraic varieties, Cambridge Tracts in Mathematics 134, Cambridge Univ. Press, 1998.

[17] R. Lazarsfeld, Positivity in algebraic geometry. I, Ergebn. Math. Grenzg. 48, Springer, 2004.

[18] G. Occhetta, A characterization of products of projective spaces, Canad. Math. Bull. 49 (2006), 270-280.

[19] V. G. SAR Kisov, On conic bundle structures, Izv. Akad. Nauk SSSR Ser. Mat. 46 (1982), 371-408; English translation: Math. USSR Izvestia 20 (1982), 355-390.

[20] T. Tsukioka, Classification of Fano manifolds containing a negative divisor isomorphic to projective space, Geom. Dedicata 123 (2006), 179-186.

[21] J. A. Wiśniewski, On contractions of extremal rays of Fano manifolds, J. reine angew. Math. 417 (1991), 141-157.

\author{
Cinzia CASAgRande \\ Dipartimento di Matematica \\ Università di Pavia \\ via Ferrata, 1 \\ 27100 Pavia - Italy \\ Current address: \\ Dipartimento di Matematica \\ Università di Torino \\ via Carlo Alberto, 10 \\ 10123 Torino - Italie \\ E-mail: cinzia.casagrande@unito.it
}

